\title{
Synthesis and application of branched type II arabinogalactans
}

Andersen, Mathias Christian Franch; Boos, Irene; Ruprecht, Colin; Willats, William G. T.; Pfrengle, Fabian; Clausen, Mads Hartvig

Published in:

Journal of organic chemistry

Link to article, DOI:

10.1021/acs.joc.7b01796

Publication date:

2017

Document Version

Peer reviewed version

Link back to DTU Orbit

Citation (APA):

Andersen, M. C. F., Boos, I., Ruprecht, C., Willats, W. G. T., Pfrengle, F., \& Clausen, M. H. (2017). Synthesis and application of branched type II arabinogalactans. Journal of organic chemistry, 82(23), 12066-12084. https://doi.org/10.1021/acs.joc.7b01796

\section{General rights}

Copyright and moral rights for the publications made accessible in the public portal are retained by the authors and/or other copyright owners and it is a condition of accessing publications that users recognise and abide by the legal requirements associated with these rights.

- Users may download and print one copy of any publication from the public portal for the purpose of private study or research.

- You may not further distribute the material or use it for any profit-making activity or commercial gain

- You may freely distribute the URL identifying the publication in the public portal 


\title{
Synthesis and application of branched type II arabinogalactans
}

\author{
Mathias C. F. Andersen ${ }^{1}$, Irene Boos ${ }^{1}$, Colin Ruprecht ${ }^{2}$, William G. T. Willats ${ }^{3}$, Fabian Pfrengle ${ }^{2,4}$, and \\ Mads H. Clausen ${ }^{1, *}$ \\ ${ }^{1}$ Center for Nanomedicine and Theranostics, Department of Chemistry, Technical University of Denmark, Kemitorvet, \\ Building 207, 2800 Kgs. Lyngby, Denmark. ${ }^{2}$ Department of Biomolecular Systems, Max-Planck-Institute of Colloids and \\ Interfaces, Am Mühlenberg 1, 14476 Potsdam, Germany. ${ }^{3}$ School of Agriculture, Food \& Rural Development, Newcastle \\ University, Newcastle upon Tyne, NE1 7RU, United Kingdom. ${ }^{4}$ Institute of Chemistry and Biochemistry, Freie Universität \\ Berlin, Arnimallee 22, 14195 Berlin, Germany.
}

*E-mail: mhc@kemi.dtu.dk

Supporting Information Placeholder
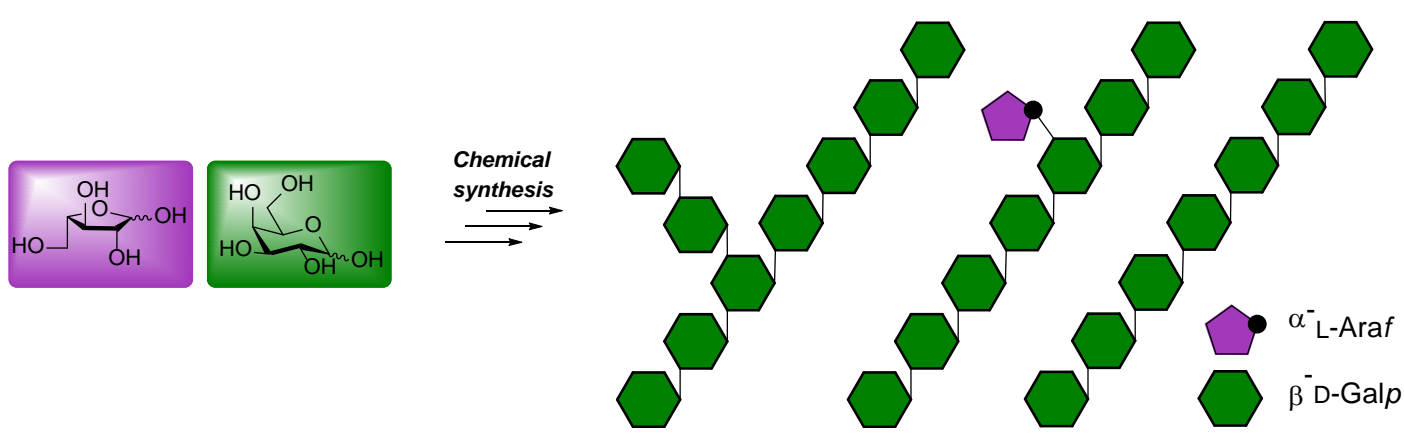

\begin{abstract}
The synthesis of linear- and $(1 \rightarrow 6)$-branched $\beta$-( $1 \rightarrow 3)$-D-galactans, structures found in plant arabinogalactan proteins (AGPs) is described. The synthetic strategy relies on iterative couplings of mono- and disaccharide thioglycoside donors, followed by a late stage glycosylation of heptagalactan backbone acceptors to introduce branching. A key finding from the synthetic study was the need to match protective groups in order to tune reactivity and ensure selectivity during the assembly. Carbohydrate microarrays were generated to enable the detailed epitope mapping of two monoclonal antibodies known to recognize AGPs: JIM16 and JIM133.
\end{abstract}

\section{Introduction.}

Arabinogalactan protein (AGP) is a widely occurring proteoglycan in plants, associated with the plasma membrane and the cell wall. AGP is one of the most complex families of macromolecules found in plants, due to a great diversity of glycans decorating the protein backbone and constituting $90-98 \%$ of the total mass. ${ }^{1}$ Although this complexity has made it impossible to address the precise function of individual AGPs, they are known to be involved in several processes in plant growth and development including differentiation, ${ }^{2}$ signaling, ${ }^{3}$ root growth, ${ }^{4}$ embryogenesis ${ }^{5}$ and programmed cell death. ${ }^{6}$ Characterization of the glycan structures of AGPs is complicated by difficulties in the isolation of single molecules and the micro-heterogeneity found in the constituent chains. Knowledge of the glycan structure is based on NMR characterization of oligosaccharide fragments or binding of monoclonal antibodies to specific carbohydrate epitopes. ${ }^{7}$ AGP glycans are predominantly type II arabinogalactan chains of 30-120 monosaccharide residues that are $\mathrm{O}$-glycosidically linked to hydroxyproline (Hyp) residues in the protein backbone. ${ }^{8}$ The type II AGs have a $\beta$ - $(1 \rightarrow 3)$-linked D-galactopyranosyl (D-Galp) backbone substituted at C-6 with side-chains of $\beta-(1 \rightarrow 6)$-linked galactose. The side chains contain a great diversity of monosaccharides including arabinofuranose, rhamnose, fucose and glucuronic acid. The AGs are generally neutral albeit some GlcpA-rich versions have been found in gum arabic. ${ }^{9}$

Knowledge of the AGP glycan backbone's biological functions is very limited. Since well-defined oligosaccharides have proven to be useful tools for elucidating protein-carbohydrate interactions (e.g. as enzyme substrates ${ }^{10}$ and for mapping the epitopes of monoclonal antibodies ${ }^{11,12}$ ), we set out to synthesize a range of linear and branched $\beta$ - $(1 \rightarrow 3)$-linked galactans (Figure 1$).{ }^{13}$ Since the AGP structure is immensely complex, there are an almost unlimited number of substructures which could be targeted for synthesis. The criteria used for selecting the structures were the following: 1) both linear and branched AGP motifs should be represented; 2) branching should occur at two different positions on the backbone; 3 ) the side-chains should contain both arabinose and galactose; 4$)$ both types of galactan branching $(\beta-(1 \rightarrow 3)$ - and $\beta$-(1 $\rightarrow 6)$-linked) should be represented. A final consideration was to ensure the structures targeted (1-7) were complimentary to the AGP previously prepared by Pfrengle and co-workers. ${ }^{13}$

We aimed to prepare a central building block 10, which could be converted to a disaccharide donor $\mathbf{1 5}$. This would lower the number of critical glycosylation reactions during the assembly of the 
larger oligosaccharides. Fenger \& Madsen showed that it is possible to glycosylate the 3-position of galactose even though the 2position is unprotected, but it was necessary to protect the 2-position after every glycosylation. ${ }^{14}$ To circumvent this, we decided to protect the 2-, 4- and 6-positions permanently and use temporary protection of the 3-position. A pivaloyl group was chosen for the 2-position since neighboring group participation was required to ensure $\beta$-selectivity. The pivaloyl group furthermore has the advantage over acetyl groups that it reduces the risk of orthoester formation, which had been a problem in the galactan synthesis by Kováć. ${ }^{15}$ However, McGill and Williams observed lower yields using a pivalyl-protected donor. ${ }^{16}$ Especially the detection of an

$$
\underbrace{}_{\mathrm{OH}} \underbrace{\mathrm{OH}}_{\mathrm{OH}}
$$

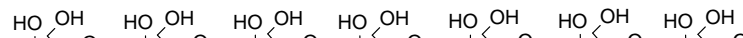
${ }_{\mathrm{OH}} \underbrace{}_{\mathrm{OH}} \underbrace{}_{\mathrm{OH}} \underbrace{}_{\mathrm{OH}} \underbrace{}_{\mathrm{OH}}$ 然 $\mathrm{HO} \mathrm{OH} \mathrm{HO} \mathrm{OH}$

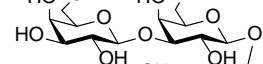
$\mathrm{HO}$ OH $\mathrm{OH}$ OH $\mathrm{OH} / \mathrm{HO}$ OH $\mathrm{HO}$ OH HO OH HO OH ${ }_{\mathrm{OH}} \underbrace{}_{\mathrm{OH}} \underbrace{}_{\mathrm{OH}} \underbrace{}_{\mathrm{OH}}$ $\alpha$-anomer and transesterification products during their reaction conditions indicate orthoester formation. Nevertheless, we decided to use the pivaloyl group in the 2-position. The 4- and 6positions were protected with a benzylidene acetal, which is easily introduced and is sterically undemanding. At first an acetyl group was chosen for temporary protection of the 3-position, as its selective removal in the presence of the more bulky pivaloyl esters has been described. ${ }^{17}$ Scheme 1 shows the building blocks required to assemble the backbone.
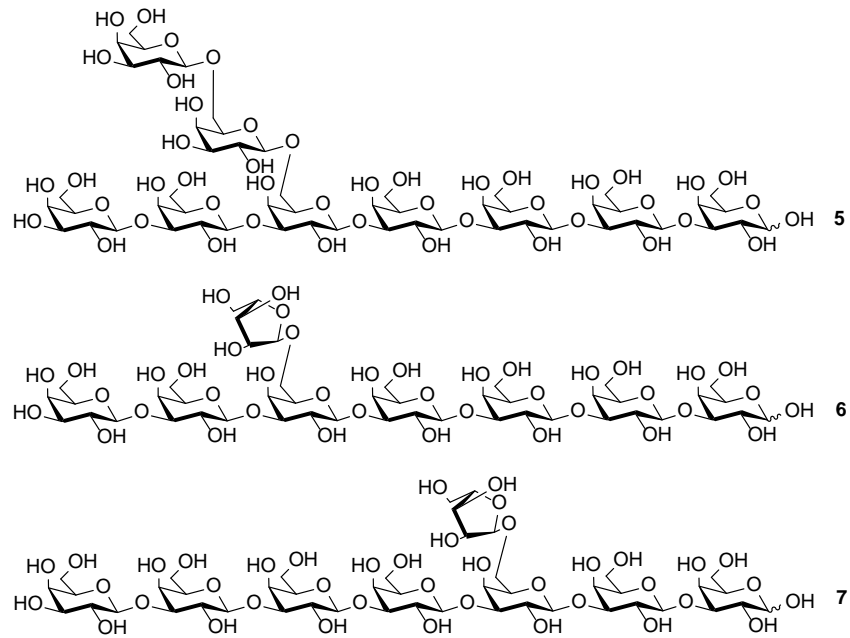

Figure 1. Target structures (1-7).
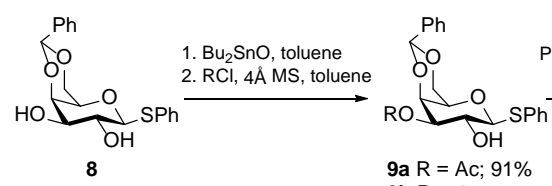
9b R $=$ CIAc; $91 \%$

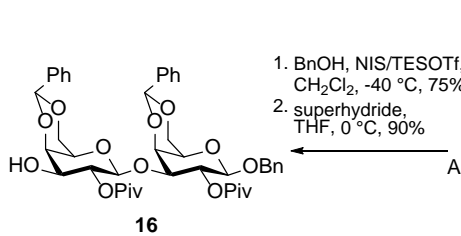

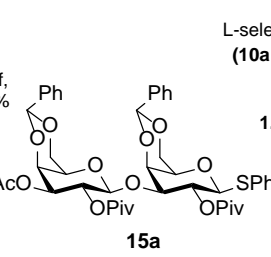

$15 a$

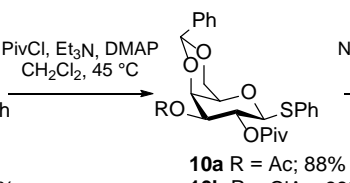

10b R $=$ CIAC; $88 \%$

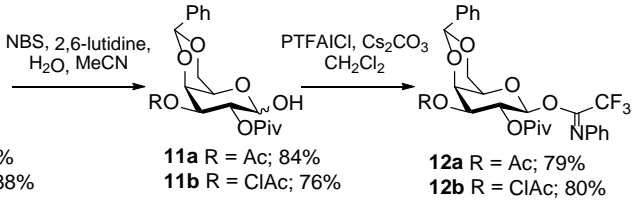

12b R $=\mathrm{ClAc} ; 80 \%$

Scheme 1. Building blocks for galactan synthesis

\section{Results and Discussion.}

Starting from the known diol $\mathbf{8}^{18}$ regioselective acetylation via formation of the stannylene acetal with $\mathrm{Bu}_{2} \mathrm{SnO}$ was performed, followed by treatment with $\mathrm{AcCl}$ and $4 \AA$ molecular sieves (Scheme 1). ${ }^{19}$ Treatment of 9a with PivCl, Et $3 \mathrm{~N}$ and DMAP afforded the fully protected thioglycoside 10a in $88 \%$ yield. In order to prepare $N$-phenyltrifluoroacetimidate (PTFAI) donor 12a, the thioglycoside had to be hydrolyzed. This turned out to be challenging due to migration of the pivaloyl group and partial hydrolysis of the benzylidene acetal. After screening several reported conditions $^{20,21}$, the use of weak bases and NBS in $\mathrm{H}_{2} \mathrm{O} / \mathrm{MeCN}$ afforded 11a in 75\% (pyridine) and 84\% yield (2,6-lutidine).

The selective deacetylation to give acceptor $\mathbf{1 4}$ turned out to be just as challenging. It was expected that the acetyl group could be removed using Zemplén conditions without affecting the pivaloyl group as shown by Andersen et al. ${ }^{18}$ However, this resulted in extensive migration as well as deprotection of the pivaloyl ester. Most likely, the pivaloyl group first migrates to the 3-position, where it is more easily accessible and is therefore subjected to transesterification. Decreasing both NaOMe concentration and temperature improved the yield to 59\%. Xu et al. had reported that $\mathrm{Mg}(\mathrm{OMe})_{2}$ can be used as a milder alternative to $\mathrm{NaOMe} .^{22}$ 
In our case, no deprotection of the pivaloyl was observed but migration persisted. The same was true for the even milder conditions using ammonia in methanol. ${ }^{23}$ Alternatively, reductive removal of the acetate was attempted. DIBAL-H did not give rise to migration but deprotection of the Piv group was observed. Similar cases had been reported by Nicolaou and co-workers. ${ }^{24}$ Gratifyingly, the more bulky reducing agents $\mathrm{LiEt}_{3} \mathrm{BH}$ (superhydride) and $\mathrm{Li}(\mathrm{sec}-\mathrm{Bu})_{3} \mathrm{BH}$ (L-selectride) provided product 14 in an excellent yield of $94 \% .^{25}$

With access to both donor 12a and acceptor 14, the disaccharide donor 15a was prepared in 92\% yield by a TMSOTf-mediated glycosylation (Scheme 1). The disaccharide 15a was converted to acceptor 16 in two steps. NIS/TESOTf-promoted glycosylation of benzyl alcohol with disaccharide 15a gave the $\beta$-benzyl glycoside in $75 \%$ yield and subsequent deacetylation with superhydride afforded $\mathbf{1 6}$ in $90 \%$ yield.

In order to access branched galactans, we envisioned to use a monosaccharide donor with a temporary C6-O protecting group and introduce this building block at either the third or fifth sugar of a backbone heptasaccharide. Starting from thioglycoside 10a, building block 13 could be prepared via a regioselective opening of the benzylidene acetal with borane-tetrahydrofuran complex and copper(II) triflate (Scheme 1). ${ }^{26}$
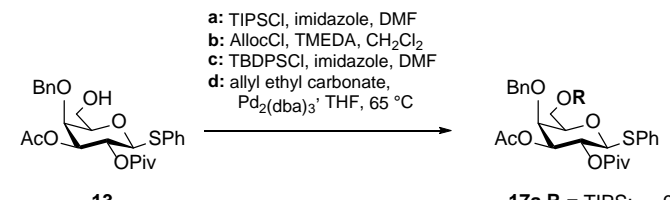

17a $\mathbf{R}=$ TIPS; $\quad 93 \%$ 17b R = TBDPS; $88 \%$ 17c R = Alloc; $91 \%$ 17d R = All; $\quad 83 \%$

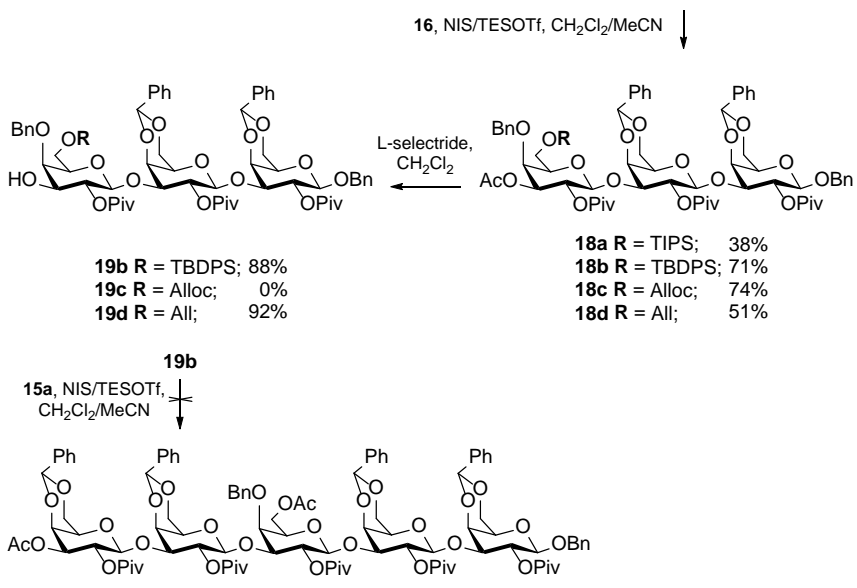

Scheme 2: Screening for a temporary $\mathrm{C6}-\mathrm{O}$ protecting group.

Next a temporary protecting group had to be found which was stable under glycosylation conditions and during reductive deacetylation. The first choice was a TIPS ether, which could be introduced to give 17a in 93\% yield (Scheme 2). ${ }^{27}$ Glycosylation of the disaccharide 16 with TIPS donor 17a resulted in several by-products and the trisaccharide 18a was isolated in $38 \%$ yield (Scheme 2). The moderate yield might have resulted from formation of the corresponding 1,6-anhydrosugar during glycosylation, previously observed for C6-O TBDMS protected donors by Bols and co-workers. $^{28}$

The TIPS group was substituted with the less acid-labile TBDPS group to prevent it from acting as an internal acceptor. Glycosylation of disaccharide $\mathbf{1 6}$ with donor $\mathbf{1 7 b}$ gave trisaccharide $\mathbf{1 8 b}$ in
$71 \%$ yield. The acetyl group was deprotected with L-selectride to give 19b, which was subjected to a glycosylation with donor 15a (Scheme 2). Unfortunately, no conversion of the acceptor was observed, presumably due to the steric demands of the TBDPS ether. Therefore, the C6-OH of $\mathbf{1 3}$ was protected with an Alloc group by reaction with allyl chloroformate and TMEDA (Scheme 2). ${ }^{29}$ Coupling of 17c to disaccharide acceptor 16 afforded trisaccharide 18c in $74 \%$ yield even though minor by-products from the reaction between NIS and the Alloc alkene were observed. Unexpectedly, the reduction with L-selectride was not selective even at low temperatures and afforded mainly the diol.

The final choice of protecting group was an allyl ether. Due to the acyl groups, it was not possible to introduce the allyl ether by Williamson ether synthesis. Instead, a method developed by Sinou and co-workers was used where allyl groups can be introduced under neutral conditions with allyl ethyl carbonate and bis(dibenzylideneacetone)palladium(0) (Scheme 2). ${ }^{30}$ This made it possible to prepare donor $\mathbf{1 7} \mathbf{d}$ in $83 \%$ yield. The glycosylation of acceptor 16 with 17d turned out to be very slow and several by-products were formed, probably from a reaction between the promotor and the alkene. Glycosylation mediated by other promotors (1-benzenesulfinyl piperidine (BSP)/ $/ \mathrm{Tf}_{2} \mathrm{O}^{31}, \mathrm{Ph}_{2} \mathrm{SO} / \mathrm{Tf}_{2} \mathrm{O}^{32}$ or $\mathrm{Me}_{2} \mathrm{~S}_{2} / \mathrm{Tf}_{2} \mathrm{O}^{33}$ with or without DTBMP) did not give significantly higher yields (33$65 \%)$.

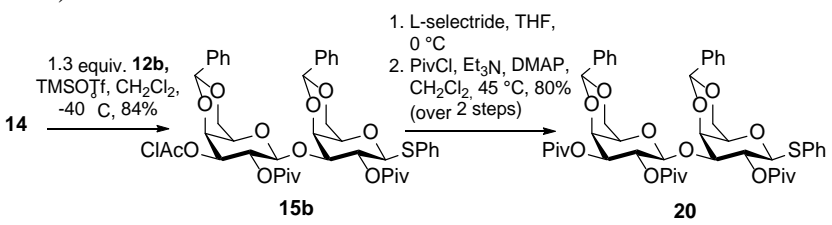

Scheme 3. Synthesis of ClAc-protected disaccharide building blocks.

Since no suitable orthogonal protecting group was found, we decided to follow a different strategy. The 3-position was temporarily protected with a chloroacetyl group. This made it possible to use an acetyl group for the 6-position, which could be removed by L-selectride reduction at the end of the backbone synthesis. The synthesis of the disaccharide donor was performed in analogy to previously described procedures. The chloroacetyl group was regioselectively introduced in the 3-position via the stannylene acetal to afford 9b in excellent yield (Scheme 1). Pivaloyl protection gave the fully protected thioglycoside $\mathbf{1 0 b}$ and hydrolysis followed by treatment with PTFAICl and $\mathrm{Cs}_{2} \mathrm{CO}_{3}$ afforded imidate $\mathbf{1 2} \mathbf{b}$. The thioglycoside $\mathbf{1 0 b}$ could be transformed to acceptor 14 by removal of the chloroacetyl group with L-selectride. 


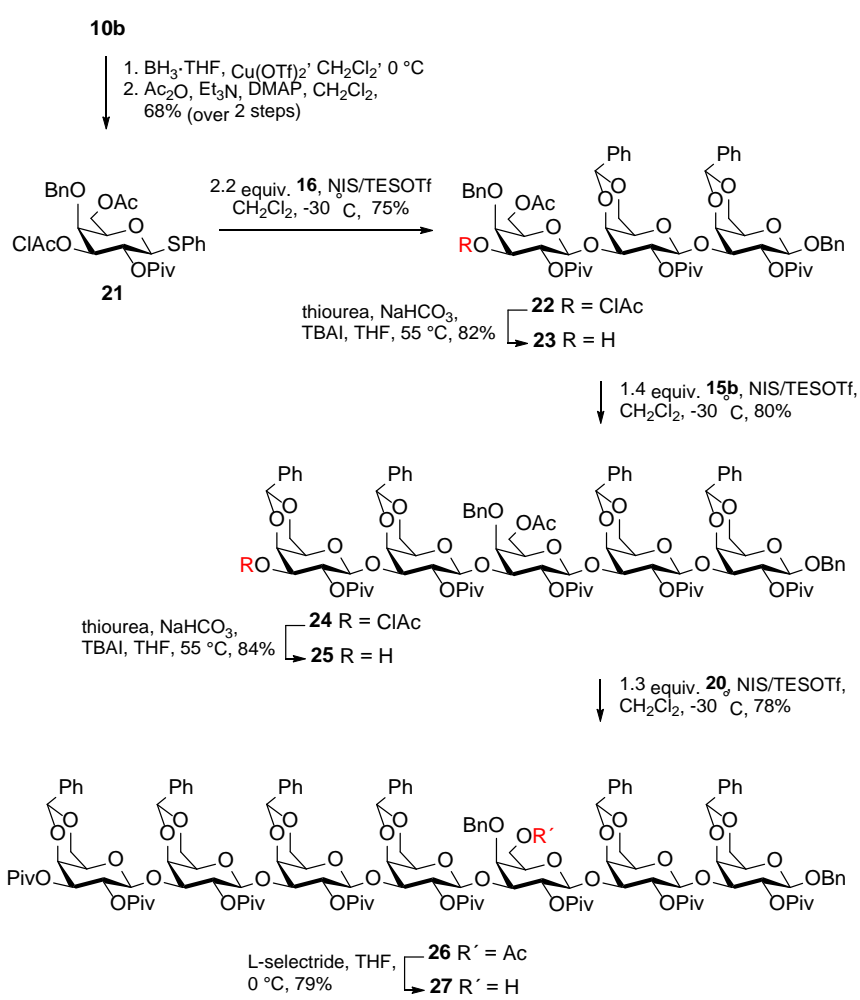

Scheme 4. Synthesis of heptasaccharide 27 with branching point at $3^{\text {rd }}$ residue.

TMSOTf-catalyzed coupling of $\mathbf{1 2 b}$ with acceptor $\mathbf{1 4}$ afforded disaccharide $\mathbf{1 5 b}$ in $84 \%$ yield (Scheme 3 ). As it would be unfavorable to have a chloroacetyl in the non-reducing end of the heptasaccharide, it was changed to Piv to give $\mathbf{2 0}$ by reduction with L-selectride followed by pivaloylation. Furthermore, the disaccharide donor $\mathbf{1 5 b}$ was converted to acceptor 16 by glycosylation of benzyl alcohol followed by deprotection of the chloroacetyl group with Lselectride (not shown).

The final building block 21 was prepared by regioselective opening of the acetal of $\mathbf{1 0 b}$ followed by acetylation (Scheme 6).

With all the building blocks in hand, it was now possible to synthesize the two heptasaccharides $\mathbf{2 7}$ and $\mathbf{3 3}$. Trisaccharide $\mathbf{2 2}$ was prepared by NIS/TESOTf-promoted coupling of 21 and 16 (Scheme 4). The new protecting group combination resulted in a yield of $75 \%$, but 2.2 equiv. of donor was required in order to obtain full conversion of the acceptor.

Trisaccharide 22 was converted to acceptor 23 by deprotection of the chloroacetyl group with thiourea, $\mathrm{NaHCO}_{3}$ and TBAI. ${ }^{34} \mathrm{~A}$ second glycosylation with disaccharide donor $\mathbf{1 5 b}$ afforded the pentasaccharide $\mathbf{2 4}$ in $\mathbf{8 0} \%$ yield. We were pleased to find that the reaction gave fewer by-products and that only 1.2 equiv. of donor was required for full conversion. The chloroacetyl group was removed to give acceptor 25, which could be glycosylated with donor $\mathbf{2 0}$ to

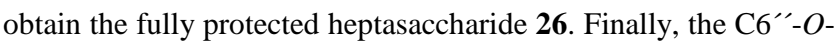
acetyl was removed selectively with L-selectride, affording acceptor 27.

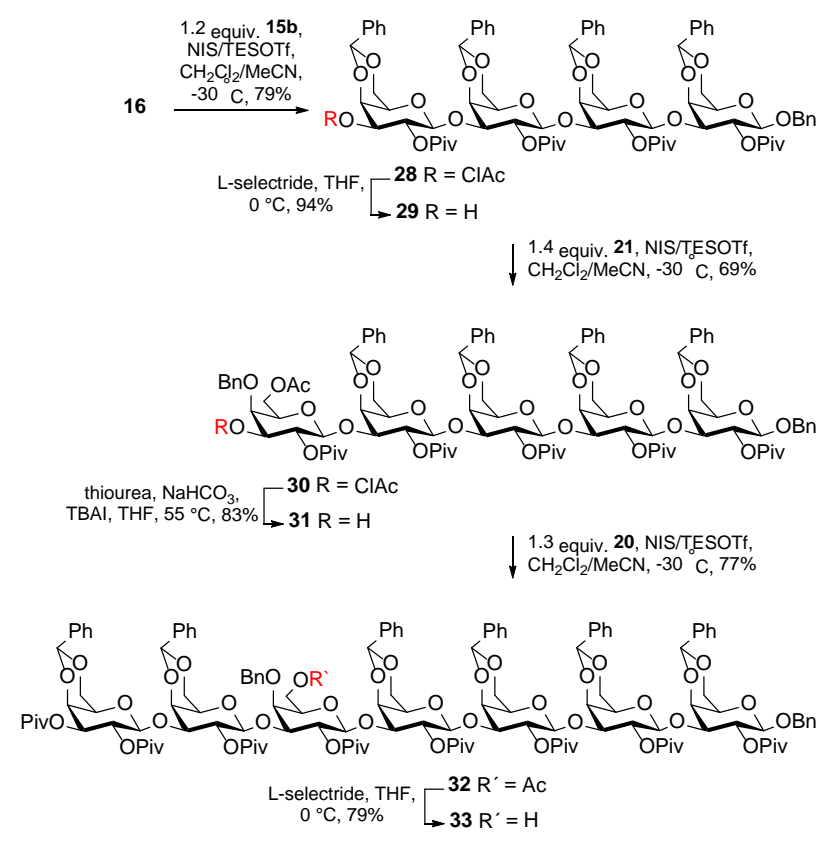

Scheme 5. Heptasaccharide synthesis with branching point at $5^{\text {th }}$ residue.

The second heptasaccharide was synthesized in a similar way (Scheme 5). NIS/TESOTf-promoted coupling of disaccharide donor $\mathbf{1 5 b}$ and acceptor $\mathbf{1 6}$ afforded tetrasaccharide $\mathbf{2 8}$ in $\mathbf{7 9 \%}$ yield. Deprotection of the chloroacetyl group gave $\mathbf{2 9}$, which was reacted with monosaccharide donor $\mathbf{2 1}$ to give pentasaccharide $\mathbf{3 0}$. The chloroacetyl group was removed and acceptor $\mathbf{3 1}$ was glycosylated with donor $\mathbf{2 0}$ to give 32. Finally, selective acetyl-deprotection afforded the second heptasaccharide acceptor 33.

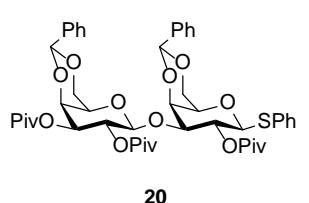

20

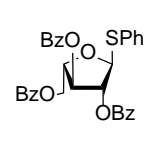

34

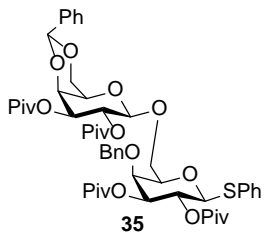

Figure 2. Donors for the synthesis of branched galactans.

Three different side chains were introduced on each heptasaccharide by glycosylation with the thioglycoside donors $20,34^{35}$ and $35^{18}$ shown in Figure 2. All of the glycosylations were promoted by NIS/TESOTf in a 1:1 mixture of $\mathrm{CH}_{2} \mathrm{Cl}_{2}$ and $\mathrm{MeCN}$ at $-30{ }^{\circ} \mathrm{C}$ (Scheme 6 and 7). The two octaasaccharides $\mathbf{3 6}$ and $\mathbf{3 8}$ and three nonasaccharides 37, 39 and $\mathbf{4 0}$ were all isolated in yields around $70 \%$.

Global deprotection of the linear penta- and heptasaccharide (24 and 26), two branched octasaccharides (36 and 38) and three nonasaccharides (37, 39 and 40) was accomplished with Et $4 \mathrm{NOH}$ and subsequent hydrogenolysis over $\mathrm{Pd}(\mathrm{OH})_{2} / \mathrm{C}$ according to Andersen et al. ${ }^{18}$

With the target oligosaccharides in hand, we demonstrated their usefulness for probing carbohydrate-protein interactions using glycan microarrays. ${ }^{36-38}$ Screening was performed essentially as was previously reported. ${ }^{12,18}$ The data for binding of the two monoclonal antibodies JIM16 ${ }^{39}$ and JIM133 ${ }^{40}$ is presented in Figure 3 and 
Table 1. Linear and branched $\beta$ - $(1 \rightarrow 4)$-linked galactans ${ }^{18}$ were included as negative controls. Interestingly, JIM133 bound to all immobilized glycans having a $\beta-(1 \rightarrow 3)$ backbone and the binding intensity was not influenced by branching. In contrast, JIM16 is a much more discriminating antibody, requiring branching for binding and selective for the types of glycans found in the 6-position: the $\mathrm{mAb}$ recognizes $\beta-(1 \rightarrow 3)-\mathrm{Gal}_{2}$ substitution (compounds 3 and 4), but not Ara or $\beta-(1 \rightarrow 6)-G_{2}$ branching (compounds 5-6). This result also demonstrates the value of including two different types of digalactan branching, as the antibody was clearly able to differentiate. As such, JIM133 is useful in e.g. immunofluorescence microscopy for localizing $\beta$ - $(1 \rightarrow 3)$-linked galactan in plant tissue. 41,42 JIM16, on the other hand, is a much more selective antibody, which will help pin-point AGP substructures containing branching of $\beta$-(1 $\rightarrow 3)$-galactan. ${ }^{43}$

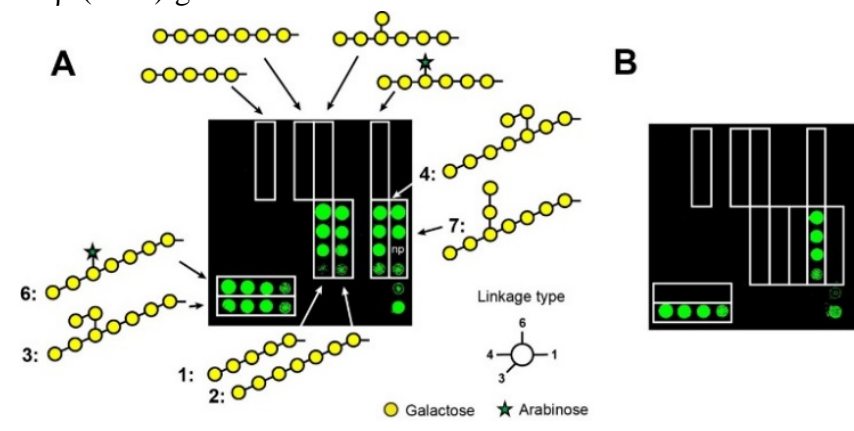

Figure 3. Binding of JIM133 and JIM16 to the synthetic oligosaccharides. (A) Fluorescence scan after incubation of the glycan microarray with JIM133. The printing pattern of the compounds is indicated using pictograms of the oligosaccharides (see legend for linkage type of the corresponding next monosaccharide). Each compound was printed in four concentrations $(200 \mu \mathrm{M}, 50 \mu \mathrm{M}$, $12.5 \mu \mathrm{M}$, and $3.1 \mu \mathrm{M}$ ); np: not printed. Oligosaccharide 7 was not included due to limited material. (B) Fluorescence scan after incubation of the glycan microarray with JIM16. Compounds were printed as indicated in (A).

Table 1. Microarray data for binding of JIM133 and JIM16 to immobilized, synthetic galactans.

\begin{tabular}{lrrrrrrrrrr}
\hline Cmp. & $\mathbf{1}$ & $\mathbf{2}$ & $\mathbf{3}$ & $\mathbf{4}$ & $\mathbf{5}$ & $\mathbf{6}$ & $\begin{array}{r}1,4- \\
\mathrm{Gal}_{5}\end{array}$ & $\begin{array}{l}1,4- \\
\mathrm{Gal}_{7}\end{array}$ & $\begin{array}{l}\text { 6’’'-Gal } \\
1,4-\mathrm{Gal}_{7}\end{array}$ & $\begin{array}{l}\text { 6’’’Ara } \\
1,4-\mathrm{Gal}_{7}\end{array}$ \\
\hline JIM133 & $71^{\mathrm{a}}$ & 43 & 26 & 43 & 59 & 60 & 0 & 0 & 0 & 0 \\
\hline JIM16 & 0 & 0 & 100 & 63 & 0 & 0 & 0 & 0 & 0 & 0 \\
\hline
\end{tabular}

${ }^{a}$ Values are normalized signal intensities (relative to the signal from JIM16 binding to immobilized 3). 


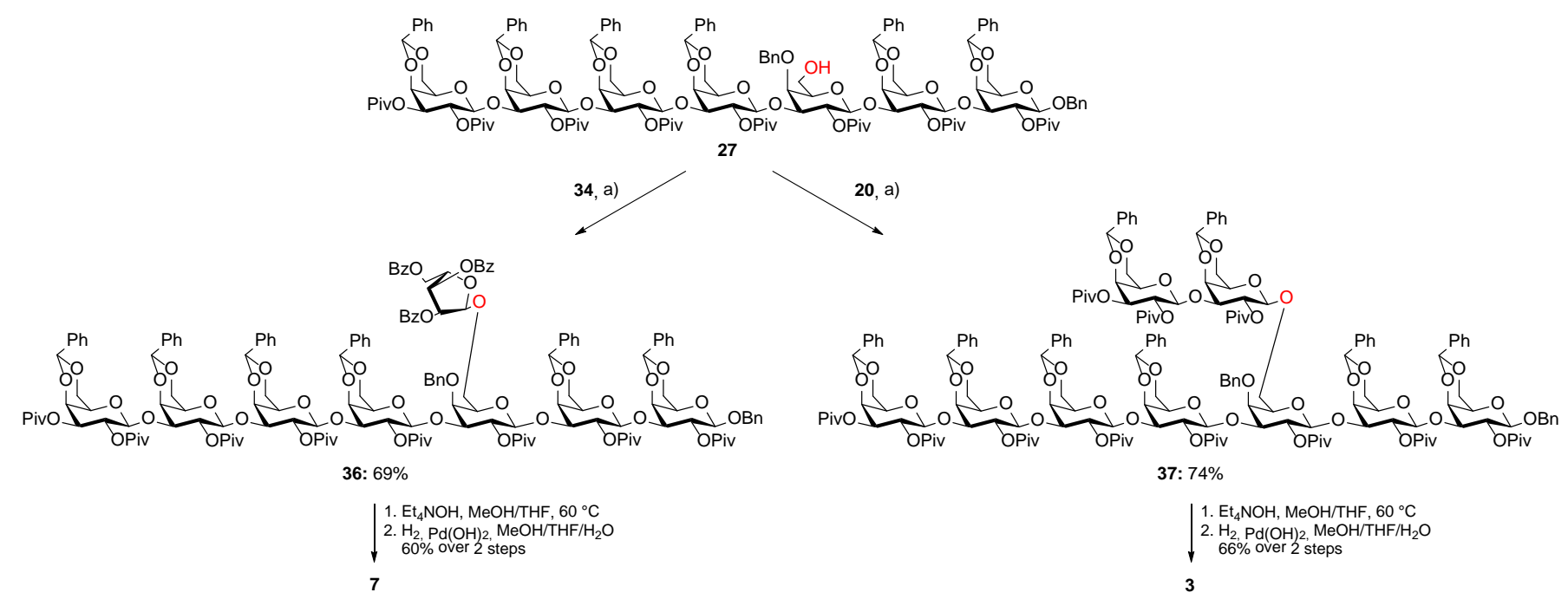

Scheme 6: Synthesis and deprotection of oligosaccharides with branching point at the $3^{\text {rd }}$ residue.

a) 1.5 equiv. donor, NIS/T⿱EESOTf,

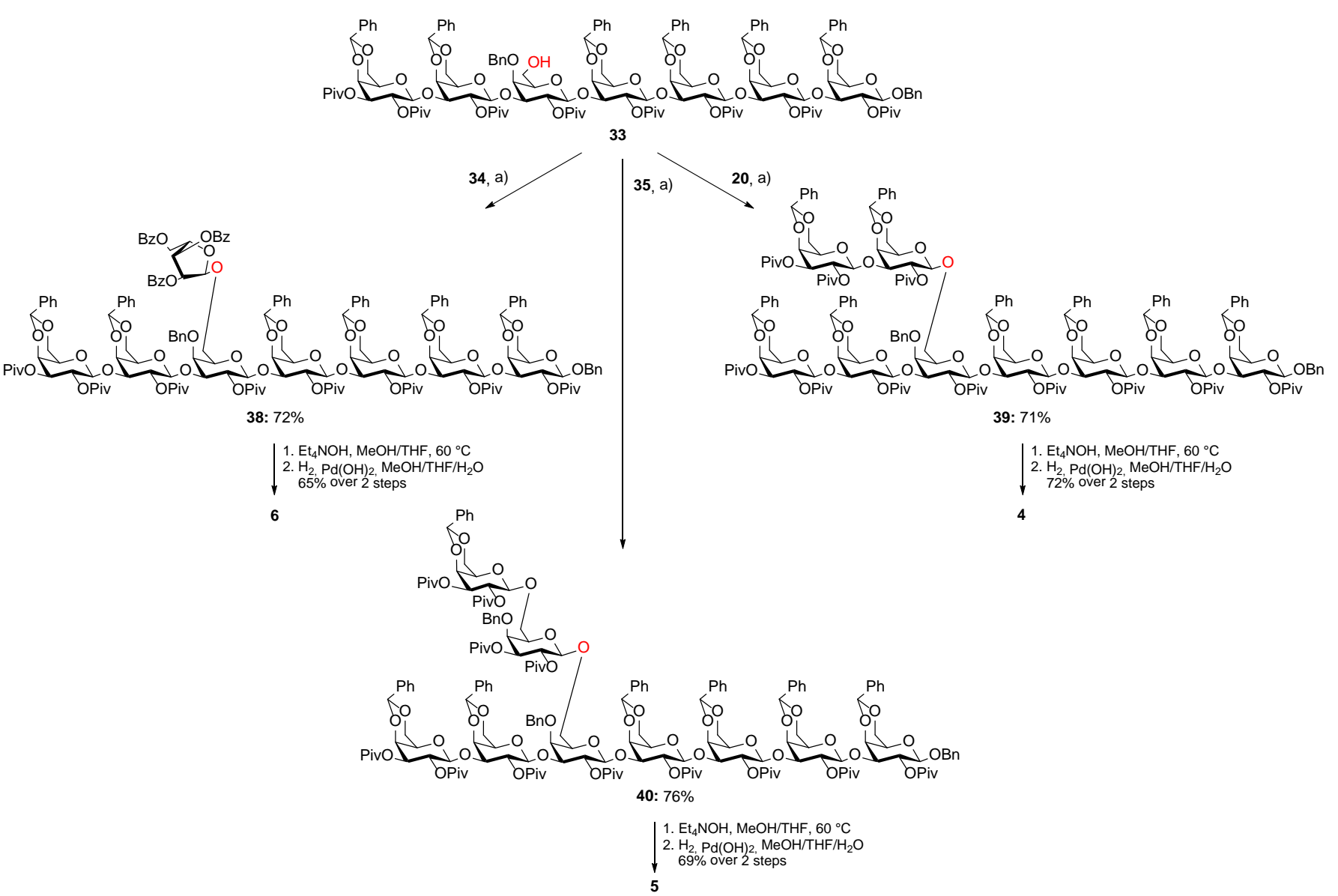

a) 1.5 equiv. donor, NIS/TESOTf, $\mathrm{CH}_{2} \mathrm{Cl}_{2} / \mathrm{MeCN},-30^{\circ} \mathrm{C}$

\section{Scheme 7: Glycosylation at $5^{\text {th }}$ residue and following deprotection.}

In conclusion, a convergent synthetic strategy for $(1 \rightarrow 6)$-branched $\beta-(1 \rightarrow 3)$-D-galactans was developed and used to prepare octa- and nonasaccharides. The substrates have been printed as oligosaccharide microarrays to characterize the epitopes of two plant cell wall- 
directed mAbs. In the future, these well-defined glycans are expected to yield new insight into the structure and function of arabinogalactan proteins in plants.

\section{EXPERIMENTAL SECTION}

General. All commercial reagents were used as obtained commercially unless otherwise noted. The dry solvents were obtained from Innovative Technology PS-MD-7 Pure-solv solvent purification system. All of the reactions were carried out in flame-dried glassware under inert atmosphere. Thin-layer chromatography (TLC) was performed on Merck Aluminium Sheets pre-coated with silica, C-60 F254 plates. Compounds were visualized by charring after dipping in CAM stain $\left(\mathrm{Ce}\left(\mathrm{SO}_{4}\right)_{2}(1.6 \mathrm{~g})\right.$ and $\left(\mathrm{NH}_{4}\right)_{6} \mathrm{Mo}_{7} \mathrm{O}_{24}(4 \mathrm{~g})$ in $10 \%$ sulphuric acid $(200 \mathrm{~mL})$. Eluent systems are specified for each Rf-value, and ratios are given as volume ratios.

Evaporation of solvents was performed with a VWR International Laborota 400 under reduced pressure (in vacuo) at temperatures ranging between $35-55^{\circ} \mathrm{C}$. Trace solvent was removed by under reduced pressure by means of an oil pump. Flash chromatography was performed using Matrex $60 \AA$ silica gel $(35-70 \mu \mathrm{m})$ as the stationary phase by the general procedure developed by Still et al. The eluent system is specified under the protocol for each synthesis. Eluent ratios are given as volume ratios.

NMR-spectras were recorded at $25{ }^{\circ} \mathrm{C}$ on a Bruker Ascend 400, Bruker Avance 800 MHz, Varian Mercury 300 B, Bruker DQX 400 and Bruker AC 500 spectrometer. Chemical shifts $(\delta)$ are reported in ppm and coupling constants in $\mathrm{Hz}$. Solvents were $\mathrm{CDCl}_{3}$, $\mathrm{CD}_{3} \mathrm{OD}, \mathrm{D}_{2} \mathrm{O}$ or $\mathrm{d}_{6}-\mathrm{DMSO}$ and their resonances were used as internal standards. For $\mathrm{D}_{2} \mathrm{O}$, the reference was 4.77 , corresponding to the HDO signal at $25{ }^{\circ} \mathrm{C} .{ }^{44}$

IR analysis was done on a Bruker Alpha-P FT-IR instrument where solid compound is applied directly onto the instrument. Optical rotation was measured on a Perkin Elmer Model 241 Polarimeter. Solvents used were either $\mathrm{CHCl}_{3}$ or $\mathrm{H}_{2} \mathrm{O}$.

High-resolution LC-DAD-MS was performed in an Agilent 1100 system equipped with a photodiode array detector (DAD) and coupled to a LCT orthogonal time-of-flight mass spectrometer (Waters-Micromass, Manchester, UK) with Z-spray electrospray ionization (ESI) source and a LockSpray probe and controlled MassLynx 4.0 software. LC-MS calibration from m/z 100-900 was done with a PEG mixture. Standard separation involved a LUNA 2 column with a MeCN (50 ppm TFA) in water gradient starting from $15 \%$ to $100 \%$ over 25 minutes with a flow rate of $0.3 \mathrm{~mL} / \mathrm{min}$. High-resolution MALDI-MS was recorded using a Bruker Solarix XR 7T ESI/MALDI-FT-ICR-MS run in MALDI+ mode, externally calibrated with NaTFA cluster ions and using dithranol as the matrix.

Most of the compounds have been characterized by NMR and/or HRMS. However, $N$-phenyltrifluoroacetimidates are generally not characterized due to low stability.

Phenyl 3-O-acetyl-4,6- $O$-benzylidene-1-thio- $\beta$-D-galactopyranoside (9a). Di-n-butyl tin oxide (36.3 g; $145.7 \mathrm{mmol}$ ) was added to a solution of compound 8 (50.0 g, $138.7 \mathrm{mmol})$ in dry toluene $(1000 \mathrm{~mL})$ and stirred under refluxing temperature for $12 \mathrm{~h}$. The reaction mixture was cooled to $0{ }^{\circ} \mathrm{C}$, and freshly activated $4 \AA \mathrm{MS}$ (50 g) were added. After $30 \mathrm{~min}$ acetyl chloride $(10.2 \mathrm{~mL}$, $142.89 \mathrm{mmol}$ ) were added drop wise and stirring was maintained at this temperature for $1 \mathrm{~h}$. The reaction was quenched by addition of $\mathrm{MeOH}$, filtered through a pad of celite and concentrated. The crude product was purified by flash chromatography (4:1 toluene/EtOAc) to give 9a as white solid. $\mathrm{R}_{\mathrm{f}} 0.12$ (9:1 Tol/EtOAc). Yield: $49.72 \mathrm{~g}$ (91\%). ${ }^{1} \mathbf{H}$ NMR $\left(400 \mathrm{MHz}, \mathrm{CDCl}_{3}\right) \delta 7.65-7.58\left(\mathrm{~m}, 2 \mathrm{H}, \mathrm{H}^{\mathrm{SPh}}\right)$, $7.37-7.15\left(\mathrm{~m}, 8 \mathrm{H}, \mathrm{H}^{\mathrm{SPh}}, \mathrm{H}^{\mathrm{Bn}}\right), 5.39\left(\mathrm{~s}, 1 \mathrm{H},-\mathrm{CH}^{\text {benzylidene }}\right), 4.83(\mathrm{dd}$, $\left.J_{2,3}=9.8, J_{3,4}=3.4 \mathrm{~Hz}, 1 \mathrm{H}, \mathrm{H}-3\right), 4.51$ (d, $\left.J_{1,2}=9.8 \mathrm{~Hz}, 1 \mathrm{H}, \mathrm{H}-1\right)$, 4.31 (m, 1H, H-4), 4.29 (dd, J6a,6b $=12.5, J_{5,6 a}=1.3 \mathrm{~Hz}, 1 \mathrm{H}, \mathrm{H}-6 \mathrm{a}$ ),
3.93 (dd, $J_{6 a, 6 b}=12.5, J_{5,6 b}=1.7 \mathrm{~Hz}, 1 \mathrm{H}, \mathrm{H}-6 \mathrm{~b}$ ), 3.89 (td, $J_{1,2}=J_{2,3}$ $\left.=9.8, J_{2, \mathrm{OH}}=1.9 \mathrm{~Hz}, 1 \mathrm{H}, \mathrm{H}-2\right), 3.52(\mathrm{~m}, 1 \mathrm{H}, \mathrm{H}-5), 2.01\left(\mathrm{~s}, 3 \mathrm{H},-\mathrm{CH}_{3}\right.$ Ac). ${ }^{13} \mathrm{C}$ NMR $\left(101 \mathrm{MHz}, \mathrm{CDCl}_{3}\right) \delta 171.1,137.8,133.8(2 \mathrm{C})$, 130.4, 129.2, 129.1 (2C), 128.4, 128.3 (2C), 126.5 (2C), 101.1, 87.6, 75.0, 73.7, 70.0, 69.3, 65.7, 21.2. HRMS (ESI-TOF) m/z: [M $\left.+\mathrm{NH}_{4}\right]^{+}$Calcd for $\mathrm{C}_{21} \mathrm{H}_{26} \mathrm{NO}_{6} \mathrm{~S} 420.1481$ Found 420.1478 .

Phenyl 3-O-acetyl-4,6-O-benzylidene-2-O-pivaloyl-1-thio- $\beta$-Dgalactopyranoside (10a). Compound 9a (30 g; $74.54 \mathrm{mmol}$ ) was dissolved in $\mathrm{CH}_{2} \mathrm{Cl}_{2}(500 \mathrm{~mL})$. $\mathrm{Et}_{3} \mathrm{~N}$ (20.9 mL; $\left.149.1 \mathrm{mmol}\right)$, DMAP (4.55 g; $37.3 \mathrm{mmol}$ ) and pivaloyl chloride (9.5 mL; 111.8 mmol) was added to the solution and the reaction mixture was heated to $45^{\circ} \mathrm{C}$ for $4 \mathrm{~h}$. The reaction mixture was cooled to $0{ }^{\circ} \mathrm{C}$, quenched with $\mathrm{MeOH}(10 \mathrm{~mL})$, washed with water $(2 \times 500 \mathrm{~mL})$, dried over $\mathrm{MgSO}_{4}$ and concentrated. The product was purified by flash chromatography (Tol/EtOAc 15:1) to afford 10a as a white powder. Rf 0.44 (9:1 Tol/EtOAc). Yield 32.0 g (88\%). IR (neat, $\mathrm{cm}^{-1}$ ): 3061.41, 2975.48, 2872.21, 1746.23, 1479.26, 1458.20, 1440.23, 1369.52, 1276.88, 1232.47, 1171.24, 1144.91, 1093.84, 1048.73, 1025.24. ${ }^{1} \mathbf{H}$ NMR $\left(400 \mathrm{MHz}, \mathrm{CDCl}_{3}\right) \delta 7.59-7.43(\mathrm{~m}$, $\left.2 \mathrm{H}, \mathrm{H}^{\mathrm{SPh}}\right), 7.38$ - $7.26\left(\mathrm{~m}, 5 \mathrm{H}, \mathrm{H}^{\mathrm{SPh}}, \mathrm{Ar}-\mathrm{H}\right), 7.25-7.14(\mathrm{~m}, 3 \mathrm{H}, \mathrm{Ar}-$ $\mathrm{H}), 5.40$ (s, $\left.1 \mathrm{H}, \mathrm{CH}^{\text {benzylidene }}\right), 5.29$ (t, $\left.J_{1,2}=J_{2,3}=9.9 \mathrm{~Hz}, 1 \mathrm{H}, \mathrm{H}-2\right)$, $5.01\left(\mathrm{dd}, J_{2,3}=9.9, J_{3,4}=3.4 \mathrm{~Hz}, 1 \mathrm{H}, \mathrm{H}-3\right), 4.67\left(\mathrm{~d}, J_{1,2}=9.9 \mathrm{~Hz}\right.$, $1 \mathrm{H}, \mathrm{H}-1$ ), 4.30 (dd, $J_{6 a, 6 b}=12.5, J_{5,6 a}=1.6 \mathrm{~Hz}, 1 \mathrm{H}, \mathrm{H}-6 \mathrm{a}$ ), 4.27 (dd, $\left.J_{3,4}=3.4, J_{4,5}=0.9 \mathrm{~Hz}, 1 \mathrm{H}, \mathrm{H}-4\right), 3.96$ (dd, $J_{6 a, 6 b}=12.4, J_{5,6 b}=1.7$ $\mathrm{Hz}, 1 \mathrm{H}, \mathrm{H}-6 \mathrm{~b}), 3.52$ (m, 1H, H-5), 1.93 (s, 3H, - $\mathrm{CH}_{3}{ }^{\mathrm{Ac}}$ ), 1.14 (s, $\left.9 \mathrm{H}, 3 \mathrm{xCH}_{3}{ }^{\mathrm{Piv}}\right) .{ }^{13} \mathrm{C}$ NMR $\left(101 \mathrm{MHz}, \mathrm{CDCl}_{3}\right) \delta 176.3,170.6,137.5$, 133.6 (2C), 131.6, 129.1, 128.8 (2C), 128.1 (2C), 128.1, 126.5 (2C), 101.0, 85.5, 73.7, 73.0, 69.7, 69.1, 66.2, 38.7, 27.1, 20.8 (3C). HRMS (ESI-TOF) m/z: $[\mathrm{M}+\mathrm{Na}]^{+}$Calcd for $\mathrm{C}_{26} \mathrm{H}_{30} \mathrm{NaO}_{7} \mathrm{~S}$ 509.1610; Found 509.1595.

3-O-Acetyl-4,6-O-benzylidene-2-O-pivaloyl-D-galactopyranose (11a). Compound 10a (20.0 g; $41.1 \mathrm{mmol})$ was dissolved in $\mathrm{MeCN}$ $(270 \mathrm{~mL})$ and water $(30 \mathrm{~mL})$. NBS (29.3 g; $164.4 \mathrm{mmol})$ and 2,6lutidine (23.8 mL; $205.5 \mathrm{mmol})$ were added and the reaction was stirred at $50{ }^{\circ} \mathrm{C}$ until TLC showed full conversion $(2 \mathrm{~h})$. The solution was diluted with $\mathrm{CH}_{2} \mathrm{Cl}_{2}(500 \mathrm{~mL})$ and washed with sat. aq. $\mathrm{NaS}_{2} \mathrm{O}_{3}(200 \mathrm{~mL})$ and sat. aq. $\mathrm{NaHCO}_{3}(200 \mathrm{~mL})$. The organic phase was dried over $\mathrm{MgSO}_{4}$, filtered and concentrated. The product was purified by flash chromatography (6:1 Tol/EtOAc) to afford 11a as $\alpha / \beta$ mixture. $\mathrm{R}_{\mathrm{f}} 0.12$ (9:1 Tol/EtOAc). Yield: $17.2 \mathrm{~g}$ (84\%). IR (neat, $\mathrm{cm}^{-1}$ ): 3468.40, 2974.49, 2934.27, 2909.50, 2873.74, 1737.61, 1479.91, 1457.72, 1369.67, 1240.78, 1115.19, 1093.82, 1025.30, 977.04. ${ }^{1} \mathbf{H}$ NMR $\left(400 \mathrm{MHz}, \mathrm{CDCl}_{3}\right) \delta 7.43(\mathrm{~m}$, $2 \mathrm{H}, \mathrm{Ar}-\mathrm{H}), 7.30$ (m, 3H, Ar-H), 6.31 (d, $\left.J_{1,2}=3.6 \mathrm{~Hz}, 1 \mathrm{H}, \mathrm{H}-1\right)$, 5.45 (s, $\left.1 \mathrm{H}, \mathrm{H}^{\text {benzylidene }}\right), 5.08$ (dd, $J_{2,3}=10.5, J_{3,4}=3.4 \mathrm{~Hz}, 1 \mathrm{H}, \mathrm{H}-$ 3), 4.42 (dd, $J_{3,4}=3.4, J_{4,5}=1.2 \mathrm{~Hz}, 1 \mathrm{H}, \mathrm{H}-4$ ), 4.35 (dd, $J_{2,3}=10.5$, $\left.J_{1,2}=3.6 \mathrm{~Hz}, 1 \mathrm{H}, \mathrm{H}-2\right), 4.22$ (dd, $J_{6 a, 6 b}=12.6, J_{5,6 a}=1.6 \mathrm{~Hz}, 1 \mathrm{H}, \mathrm{H}-$ 6a), 3.97 (dd, $\left.J_{6 a, 6 b}=12.6, J_{5,6 b}=1.8 \mathrm{~Hz}, 1 \mathrm{H}, \mathrm{H}-6 \mathrm{~b}\right), 3.75(\mathrm{~m}, 1 \mathrm{H}$, $\mathrm{H}-5), 2.09$ (s, 3H, $\mathrm{CH}_{3}{ }^{\mathrm{Ac}}$ ), 1.20 (s, 9H, $\left.3 \mathrm{xCH}_{3}{ }^{\mathrm{Piv}}\right) .{ }^{13} \mathrm{C}$ NMR (101 $\left.\mathrm{MHz} \mathrm{CDCl}_{3}\right) \delta 177.0,171.7,137.5,129.2,128.4,126.3,100.9$, 92.8, 73.8, 71.7, 69.1, 66.0, 64.7, 39.5, 27.3, 21.2. HRMS (ESITOF) $\mathrm{m} / \mathrm{z}:[\mathrm{M}+\mathrm{Na}]^{+}$Calcd for $\mathrm{C}_{20} \mathrm{H}_{26} \mathrm{NaO}_{8}$ : 417.1525; Found 417.1529.

3-O-Acetyl-4,6-O-benzylidene-2-O-pivaloyl- $\beta$-D-galactopyranose $N$-phenyl trifluoroacetimidate (12a). Compound 11a (13 g; $33.0 \mathrm{mmol}$ ) was dissolved in $\mathrm{CH}_{2} \mathrm{Cl}_{2}(300 \mathrm{~mL})$ and cooled to $0{ }^{\circ} \mathrm{C}$. $\mathrm{Cs}_{2} \mathrm{CO}_{3}$ (21.5 g; $65.9 \mathrm{mmol}$ ) was added followed by $N$-phenyl trifluoroacetimidoyl chloride (13.7 g; $65.9 \mathrm{mmol})$. The ice bath was removed and the reaction mixture was stirred until TLC showed full conversion ( $5 \mathrm{~h}$ ). It was then filtered, concentrated and purified by flash chromatography (20:1 Tol/EtOAc) to give an off-white solid. Rf 0.66 (9:1 Tol/EtOAc) Yield: 14.7 g (79\%). 
Phenyl 4,6- $O$-benzylidene-2- $O$-pivaloyl-1-thio- $\beta$-D-galactopyranoside (14). Compound 10a (10 g; $20.6 \mathrm{mmol})$ was dissolved in dry $\mathrm{CH}_{2} \mathrm{Cl}_{2}(200 \mathrm{~mL})$ and cooled to $0{ }^{\circ} \mathrm{C}$. A $1 \mathrm{M} \mathrm{L}$-selectride solution in THF $(61.6 \mathrm{~mL})$ was added and the reaction was stirred at 0 ${ }^{\circ} \mathrm{C}$ until complete consumption of the starting material $(4 \mathrm{~h})$. The reaction mixture was poured into sat. aq. $\mathrm{NH}_{4} \mathrm{Cl}(400 \mathrm{~mL})$. The organic phase was dried over $\mathrm{MgSO}_{4}$, filtered and concentrated (avoid concentrating to dryness since the borane salts can be explosive). The crude product was purified by flash chromatography (9:1 Tol/EtOAc) to give an off-white solid. $\mathrm{R}_{\mathrm{f}} 0.23$ (9:1 Tol/EtOAc) Yield: 8.6 g (94\%). ${ }^{1} \mathbf{H}$ NMR $\left(400 \mathrm{MHz}, \mathrm{CDCl}_{3}\right) \delta 7.51$ (m, 2H, Ar-H), 7.38 - 7.10 (m, 10H, Ar-H), 5.45 (s, 1H, CH ${ }^{\text {benzylidene }), ~} 4.98$ $\left(\mathrm{t}, J_{1,2}=J_{2,3}=9.7 \mathrm{~Hz}, 1 \mathrm{H}, \mathrm{H}-2\right), 4.60\left(\mathrm{~d}, J_{1,2}=9.7 \mathrm{~Hz}, 1 \mathrm{H}, \mathrm{H}-1\right)$, 4.31 (dd, $J_{6 a, 6 b}=12.5, J_{5,6 a}=1.5 \mathrm{~Hz}, 1 \mathrm{H}, \mathrm{H}-6 \mathrm{a}$ ), 4.14 (dd, $J_{3,4}=3.6$, $\left.J_{4,5}=1.1 \mathrm{~Hz}, 1 \mathrm{H}, \mathrm{H}-4\right), 3.96$ (dd, $J_{6 a, 6 b}=12.5, J_{5,6 b}=1.7 \mathrm{~Hz}, 1 \mathrm{H}, \mathrm{H}-$ 6b), 3.67 (dd, $J_{2,3}=9.7, J_{3,4}=3.6 \mathrm{~Hz}, 1 \mathrm{H}, \mathrm{H}-3$ ), $3.51-3.43$ (m, $1 \mathrm{H}$, $\mathrm{H}-5), 1.19$ (s, 9H, $3 \mathrm{xCH}_{3}{ }^{\mathrm{Piv}}$ ). ${ }^{13} \mathrm{C}$ NMR $\left(101 \mathrm{MHz}, \mathrm{CDCl}_{3}\right) \delta 177.6$, 137.4, 133.6 (2C), 131.6, 129.4, 128.8 (2C), 128.2 (2C), 128.1, 126.5 (2C), 101.4, 85.1, 75.7, 72.9, 69.9, 69.5, 69.2, 38.8, 27.2 (3C). HRMS (ESI-TOF) m/z: [M + Na $]^{+}$Calcd for $\mathrm{C}_{24} \mathrm{H}_{28} \mathrm{NaO}_{6} \mathrm{~S}$ : 467.1504; Found 467.1511.

Phenyl 3-O-acetyl-4,6-O-benzylidene-2-O-pivaloyl- $\beta$-D-galactopyranosyl-( $1 \rightarrow 3$ )- 4,6 - $O$-benzylidene-2- $O$-pivaloyl-1-thio- $\beta$-Dgalactopyranoside (15a). To a $500 \mathrm{~mL}$ flame-dried flask was added 14 (7.0 g, $15.7 \mathrm{mmol})$ and the 12a (11.4 g, $20.5 \mathrm{mmol})$. The mixture was co-evaporated with toluene $(2 \times 200 \mathrm{~mL})$ and subjected to vacuum overnight. The mixture dissolved in $\mathrm{CH}_{2} \mathrm{Cl}_{2}(200 \mathrm{~mL})$ and cooled to $-40{ }^{\circ} \mathrm{C}$. TMSOTf $(0.24 \mathrm{~mL} ; 1.6 \mathrm{mmol})$ was added and the reaction mixture was stirred at $-40^{\circ} \mathrm{C}(1 \mathrm{~h})$. Et3N $(1 \mathrm{~mL})$ was added and the reaction mixture was concentrated. The crude compound was purified by flash chromatography (9:1 Tol/EtOAc) affording 15a. Rf 0.23 (9:1 Tol/EtOAc). Yield: 11.9 g (92\%). ${ }^{1} \mathbf{H}$ NMR (400 MHz, $\left.\mathrm{CDCl}_{3}\right) \delta 7.48-7.36\left(\mathrm{~m}, 6 \mathrm{H}, \mathrm{Ar}-\mathrm{H}, \mathrm{H}^{\mathrm{SPh}}\right)$, 7.27 (m, 6H, , Ar-H, $\left.\mathrm{H}^{\mathrm{SPh}}\right), 7.20-7.06$ (m, 3H, Ar-H, $\left.\mathrm{H}^{\mathrm{SPh}}\right), 5.48$ (s, $\left.1 \mathrm{H},-\mathrm{CH}^{\text {benzylidene }}\right), 5.43$ (s, $\left.1 \mathrm{H},-\mathrm{CH}^{\text {benzylidene }}\right), 5.33$ (dd, $J_{2,3}=$ 10.6, $\left.J_{1,2}=8.0 \mathrm{~Hz}, 1 \mathrm{H}, \mathrm{H}-1^{2}\right), 5.30\left(\mathrm{t}, J_{1,2}=J_{2,3}=9.8 \mathrm{~Hz}, 1 \mathrm{H}, \mathrm{H}-2^{1}\right)$, $4.85\left(\mathrm{~d}, J=8.0 \mathrm{~Hz}, 1 \mathrm{H}, \mathrm{H}-1^{2}\right), 4.82\left(\mathrm{dd}, J_{2,3}=10.6, J_{2,3}=3.6 \mathrm{~Hz}\right.$, $1 \mathrm{H}, \mathrm{H}-3^{2}$ ), 4.59 (d, $J=9.8 \mathrm{~Hz}, 1 \mathrm{H}, \mathrm{H}-1^{1}$ ), $4.33-4.14$ (m, 5H, H$\left.3^{1}, \mathrm{H}-4^{1 / 2}, \mathrm{H}-4^{1 / 2}, \mathrm{H}-6 \mathrm{a}^{1}, \mathrm{H}-6 \mathrm{a}^{2}\right), 3.98\left(\mathrm{dd}, J_{6 a, 6 b}=12.5, J_{5,6 b}\right.$ $\left.=1.8 \mathrm{~Hz}, 1 \mathrm{H}, \mathrm{H}-6 \mathrm{~b}^{1}\right), 3.92\left(\mathrm{dd}, J_{6 a, 6 b}=12.5, J_{5,6 b}=1.7 \mathrm{~Hz}, 1 \mathrm{H}, \mathrm{H}-\right.$ $6 \mathrm{~b}^{2}$ ), $3.40-3.36$ (m, $1 \mathrm{H}, \mathrm{H}-5^{1 / 2}$ ), 3.36 (s, $\left.1 \mathrm{H}, \mathrm{H}-5^{1 / 2}\right), 1.95$ (s, $3 \mathrm{H}$, $\left.\mathrm{CH}_{3}{ }^{\mathrm{Ac}}\right), 1.22$ (s, 9H, 3xCH $\left.3{ }^{\mathrm{Piv}}\right), 1.00$ (s, 9H, 3xCH$\left.{ }_{3}{ }^{\mathrm{Piv}}\right) .{ }^{13} \mathbf{C} \mathbf{~ N M R}$ $\left(101 \mathrm{MHz}, \mathrm{CDCl}_{3}\right) \delta 176.8,176.1,170.6,137.9,137.5,133.5$, 132.3 (2C), 129.1, 128.7 (2C), 128.6 (2C), 128.2 (2C), 127.9 (2C), 127.6, 126.24 (2C), 126.21, 100.8, 100.1, 99.4, 86.8, 75.9, 73.6, 73.6, 71.8, 70.2, 69.4, 68.9, 68.8, 68.2, 66.6, 38.8, 38.7, 27.4 (3C), 27.0 (3C), 20.80. HRMS (ESI-TOF) m/z: $[\mathrm{M}+\mathrm{Na}]^{+}$Calcd for $\mathrm{C}_{44} \mathrm{H}_{52} \mathrm{NaO}_{13} \mathrm{~S}$ : 843.3026; Found 843.3014.

Benzyl 3-O-acetyl-4,6-O-benzylidene-2-O-pivaloyl- $\beta$-D-galactopyranosyl-(1 $\rightarrow 3)$-4,6-O-benzylidene-2-O-pivaloyl- $\beta$-D-galactopyranoside (41). Compound 15a (2.0 g; $2.4 \mathrm{mmol})$ was dried azeotropically with toluene ( $2 \times 20 \mathrm{~mL})$ and subjected to vacuum overnight. Benzyl alcohol (0.8 g; $7.3 \mathrm{mmol})$ was added. The mixture was dissolved in dry $\mathrm{CH}_{2} \mathrm{Cl}_{2}(50 \mathrm{~mL})$ and cooled to $-40{ }^{\circ} \mathrm{C}$. NIS (602 mg; $2.7 \mathrm{mmol}$ ) and TESOTf (64 mg; $0.24 \mathrm{mmol}$ ) was added and the reaction mixture was stirred at $-40{ }^{\circ} \mathrm{C}$ until TLC revealed full conversion of the donor $(2 \mathrm{~h})$. The solution was diluted with $\mathrm{CH}_{2} \mathrm{Cl}_{2}(100 \mathrm{~mL})$ and washed with sat. aq. $\mathrm{NaS}_{2} \mathrm{O}_{3}(100 \mathrm{~mL})$ and sat. aq. $\mathrm{NaHCO}_{3}(100 \mathrm{~mL})$. The organic phase was dried over $\mathrm{MgSO}_{4}$, filtered and concentrated. The product was purified by flash chromatography (9:1 toluene/EtOAc) to afford $\mathbf{4 1}$ as an offwhite solid. $\mathrm{R}_{\mathrm{f}} 0.10$ (9:1 Tol/EtOAc) Yield: $1.5 \mathrm{~g}$ (75\%). IR (neat, $\left.\mathrm{cm}^{-1}\right)$ : 3524.57, 3065.71, 2973.85, 1733.56, 1497.34, 1479.42, 1454.83, 1398.37, 1278.46, 1249.26, 1167.19, 1139.04, 1087.54, 1060.73. ${ }^{1} \mathbf{H}$ NMR $\left(400 \mathrm{MHz}, \mathrm{CDCl}_{3}\right) \delta 7.51-7.38\left(\mathrm{~m}, 4 \mathrm{H}, \mathrm{H}^{\mathrm{SPh}}\right)$,
7.35 - 7.13 (m, 11H, $\mathrm{H}^{\mathrm{SPh}}$, Ar-H), 5.51 (s, 1H, -CH $\left.{ }^{\text {benzylidene }}\right), 5.43$ (s, $1 \mathrm{H},-\mathrm{CH}^{\text {benzylidene }}$ ), 5.42 (dd, $\left.J_{2,3}=10.4, J_{1,2}=7.9 \mathrm{~Hz}, 1 \mathrm{H}, \mathrm{H}-2\right)$, 5.35 (dd, $J_{2,3}=10.6, J_{1,2}=8.1 \mathrm{~Hz}, 1 \mathrm{H}, \mathrm{H}-2$ '), 4.93 (d, $J_{1,2}=8.1 \mathrm{~Hz}$, $1 \mathrm{H}, \mathrm{H}-1^{\prime}$ ), $4.87-4.79$ (m, 2H, H-3', $-\mathrm{CH}_{2}{ }^{\mathrm{Bn}}$ ), 4.47 (d, $J=11.9 \mathrm{~Hz}$, $\left.1 \mathrm{H},-\mathrm{CH}_{2}{ }^{\mathrm{Bn}}\right), 4.37$ (d, $\left.J=7.9 \mathrm{~Hz}, 1 \mathrm{H}, \mathrm{H}-1\right), 4.34-4.23$ (m, 4H, H4',H-4, H-6a', H-6b'), 4.13 (dd, $J_{2,3}=10.4, J_{3,4}=3.4 \mathrm{~Hz}, 1 \mathrm{H}, \mathrm{H}-3$ ), 3.99 (m, 2H, H-6a, H-6b), 3.42 - 3.26 (m, 2H, H-5,H-5'), 1.96 (s, $\left.3 \mathrm{H},-\mathrm{CH}_{3}{ }^{\mathrm{Ac}}\right), 1.10$ (s, 9H, 3xCH$\left.{ }_{3}^{\mathrm{Piv}}\right), 1.05$ (s, 9H, $\left.3 \mathrm{xCH}_{3}{ }^{\mathrm{Piv}}\right) .{ }^{13} \mathrm{C}$ NMR $\left(101 \mathrm{MHz}, \mathrm{CDCl}_{3}\right) \delta 176.8,176.1,170.7,137.8,137.5$, 137.2, 129.08, 129.05, 128.6, 128.24, 128.22, 128.20, 127.96 (2C), 127.8 (2C), 127.5, 126.3 (2C), 126.1 (2C), 100.8, 100.3, 100.2, 99.5, 75.9, 73.6, 72.7, 71.9, 70.8, 69.9, 68.83, 68.77, 68.2, 66.9, 66.7, 38.8, 38.7, 27.22, 27.19, 27.19, 27.08 (3C), 27.0 (3C), 20.8. HRMS (ESI-TOF) m/z: $\left[\mathrm{M}+\mathrm{NH}_{4}\right]^{+}$Calcd for $\mathrm{C}_{45} \mathrm{H}_{58} \mathrm{NO}_{14}$ 836.3857 Found 836.3843.

Benzyl 4,6-O-benzylidene-2- $O$-pivaloyl- $\beta$-D-galactopyranosyl$(1 \rightarrow 3)-4,6-O$-benzylidene-2- $O$-pivaloyl- $\beta$-D-galactopyranoside (16). Compound 41 (1.5 g; $1.8 \mathrm{mmol}$ ) was dissolved in dry $\mathrm{CH}_{2} \mathrm{Cl}_{2}$ (30 mL) and cooled to $0{ }^{\circ} \mathrm{C}$. A $1 \mathrm{M} \mathrm{L}$-selectride solution in THF $(5.5 \mathrm{~mL})$ was added and the reaction was stirred at $0{ }^{\circ} \mathrm{C}$ until complete consumption of the starting material $(5 \mathrm{~h})$. The reaction mixture was poured into sat. aq. $\mathrm{NH}_{4} \mathrm{Cl}(100 \mathrm{~mL})$. The organic phase was dried over $\mathrm{MgSO}_{4}$, filtered and concentrated (avoid concentrating to dryness since the borane salts can be explosive). The crude product was purified by flash chromatography (6:1 Tol/EtOAc) yielding $\mathbf{1 6}$ as an off-white solid. Rf 0.46 (2:1 Tol/EtOAc). Yield: $1.28 \mathrm{~g}$ (90\%). ${ }^{\mathbf{1}} \mathbf{H}$ NMR (400 MHz, $\left.\mathrm{CDCl}_{3}\right) \delta$ 7.51 - 7.13 (m, 15H, Ar-H), 5.49 (s, $\left.1 \mathrm{H}, \mathrm{CH}^{\text {benzylidene }}\right), 5.47$ (s, $1 \mathrm{H}$, $\mathrm{CH}^{\text {benzylidene }}$ ), 5.45 (dd, $J_{2,3}=10.1, J_{1,2}=7.9 \mathrm{~Hz}, 1 \mathrm{H}, \mathrm{H}-2^{1}$ ), 5.00 (dd, $\left.J_{2,3}=10.1, J_{1,2}=8.0 \mathrm{~Hz}, 1 \mathrm{H}, \mathrm{H}-2^{2}\right), 4.84\left(\mathrm{~d}, J_{C H 2}=11.8 \mathrm{~Hz}, 1 \mathrm{H}\right.$, $\left.\mathrm{CH}_{2}{ }^{\mathrm{Bn}}\right), 4.84\left(\mathrm{~d}, J_{1,2}=8.0 \mathrm{~Hz}, 1 \mathrm{H}, \mathrm{H}_{-1}^{2}\right), 4.48\left(\mathrm{~d}, J_{\mathrm{CH} 2}=11.9 \mathrm{~Hz}\right.$, $\left.1 \mathrm{H}, \mathrm{CH}_{2}{ }^{\mathrm{Bn}}\right), 4.39$ (d, $\left.J_{1,2}=7.9 \mathrm{~Hz}, 1 \mathrm{H}, \mathrm{H}^{1}{ }^{1}\right), 4.31-4.23(\mathrm{~m}, 3 \mathrm{H}$, $\mathrm{H}-4^{1}, \mathrm{H}-6 \mathrm{a}^{2}, \mathrm{H}-6 \mathrm{~b}^{2}$ ), 4.10 (d, $J_{3,4}=3.7 \mathrm{~Hz}, 1 \mathrm{H}, \mathrm{H}-4^{2}$ ), 4.08 (dd, $J_{2,3}$ $\left.=10.1, J_{3,4}=3.2 \mathrm{~Hz}, 1 \mathrm{H}, \mathrm{H}-3^{1}\right), 4.02-3.95\left(\mathrm{~m}, 2 \mathrm{H}, \mathrm{H}-6 \mathrm{a}^{1}, \mathrm{H}-6 \mathrm{~b}^{1}\right)$, 3.52 (dd, $\left.J_{2,3}=10.1, J_{3,4}=3.7 \mathrm{~Hz}, 1 \mathrm{H}, \mathrm{H}-3^{2}\right), 3.34\left(\mathrm{~s}, 1 \mathrm{H}, \mathrm{H}-5^{1}\right.$ ), 3.33 (s, $1 \mathrm{H}, \mathrm{H}-5^{2}$ ), 1.10 (s, 9H, 3xCH${ }_{3}^{\mathrm{Piv}}$ ), 1.10 (s, 9H, 3xCH${ }^{\mathrm{Piv}}$ ). ${ }^{13}$ C NMR (101 MHz, $\left.\mathrm{CDCl}_{3}\right) \delta 179.0,176.2,137.9,137.5,137.3$, 129.3, 129.1, 128.8, 128.4, 128.38, 128.34 (2C), 128.0, 127.9 (2C), 127.6 (2C), 126.4 (2C), 126.3, 101.3, 100.5, 100.4, 99.5, 76.1, 75.8, 73.2, 72.3, 72.2, 71.0, 70.0, 68.9, 67.0, 66.9, 39.0, 38.8, 27.3 (3C), 27.1 (3C). HRMS (ESI-TOF) m/z: $\left[\mathrm{M}+\mathrm{NH}_{4}\right]^{+}$Calcd for $\mathrm{C}_{43} \mathrm{H}_{56} \mathrm{NO}_{13} 794.3752$ Found 794.3756 .

Phenyl 3-O-acetyl-4- $O$-benzyl-2- $O$-pivaloyl-1-thio- $\beta$-D-galactopyranoside (13). A $1 \mathrm{~m}$ solution of $\mathrm{BH}_{3}$. THF complex in THF (61.6 mL) was added to a solution of 10a (6 g; $12.33 \mathrm{mmol})$ in $\mathrm{CH}_{2} \mathrm{Cl}_{2}(50 \mathrm{~mL})$ at $0{ }^{\circ} \mathrm{C}$. The mixture was stirred for $10 \mathrm{~min}$, and freshly dried $\mathrm{Cu}(\mathrm{OTf})_{2}$ (669 $\mathrm{mg}, 1.85 \mathrm{mmol}$ ) was added to the solution. After stirring for a $5 \mathrm{~h}$, the mixture was cooled to $0{ }^{\circ} \mathrm{C}$, and the reaction was quenched by addition of $\mathrm{Et}_{3} \mathrm{~N} \quad(1.7 \mathrm{~mL}$, $12.33 \mathrm{mmol}$ ) and methanol (30 mL, caution: hydrogen gas was evolved). The resultant mixture was concentrated at reduced pressure followed by coevaporation with methanol. The residue was purified by flash chromatography (9:1 Tol/EtOAc). Rf 0.15 (9:1 Tol/EtOAc). Yield: $4.79 \mathrm{~g}(80 \%) .{ }^{1} \mathbf{H}$ NMR (400 MHz, $\left.\mathrm{CDCl}_{3}\right) \delta$ $7.45-7.35\left(\mathrm{~m}, 2 \mathrm{H}, \mathrm{H}^{\mathrm{SPh}}\right), 7.32-7.14\left(\mathrm{~m}, 8 \mathrm{H}, \mathrm{H}^{\mathrm{SPh}}, \mathrm{H}^{\mathrm{Bn}}\right), 5.36(\mathrm{dd}$, $J_{1,2}=J_{2,3}=10.0 \mathrm{~Hz}, 1 \mathrm{H}, \mathrm{H}-2$ ), $5.00\left(\mathrm{dd}, J_{2,3}=10.0, J_{3,4}=3.0 \mathrm{~Hz}\right.$, $1 \mathrm{H}, \mathrm{H}-3), 4.68\left(\mathrm{~d}, J_{\mathrm{CH} 2}=11.7 \mathrm{~Hz}, 1 \mathrm{H},-\mathrm{CH}_{2}{ }^{\mathrm{Bn}}\right), 4.65\left(\mathrm{~d}, J_{1,2}=10.0\right.$ $\mathrm{Hz}, 1 \mathrm{H}, \mathrm{H}-1$ ), 4.44 (d, $J_{\mathrm{CH} 2}=11.7 \mathrm{~Hz}, 1 \mathrm{H},-\mathrm{CH}_{2}{ }^{\mathrm{Bn}}$ ), 3.86 (dd, $J_{3,4}=$ 3.0, $J_{4,5}=1.0 \mathrm{~Hz}, 1 \mathrm{H}$ ), 3.77 (ddd, $J_{6 a, 6 b}=11.0, J_{5,6 a}=6.7, J_{6 a, O H}=3.9$ Hz, 1H, H-6a), 3.59 - 3.50 (m, 1H, H-5), 3.48 (ddd, $J_{6 a, 6 b}=11.1$, $\left.J_{5,6 b}=8.6, J_{6 b, \mathrm{OH}}=5.2 \mathrm{~Hz}, 1 \mathrm{H}, \mathrm{H}-6 \mathrm{~b}\right), 1.92$ (s, 3H, $\left.-\mathrm{CH}_{3} \mathrm{Ac}\right), 1.14$ (s, 9H, 3xCH 3 Piv). ${ }^{13} \mathrm{C}$ NMR (101 MHz, $\left.\mathrm{CDCl}_{3}\right) \delta 176.7,170.2$, 137.5, 133.2, 132.0 (2C), 128.9 (2C), 128.5 (2C), 128.3 (2C), $128.1,127.8,86.9,78.9,75.0,74.7,73.8,67.5,61.8,38.8,27.0$ 
(3C), 20.8. HRMS (ESI-TOF) m/z: $\left[\mathrm{M}+\mathrm{NH}_{4}\right]+$ Calcd for $\mathrm{C}_{26} \mathrm{H}_{36} \mathrm{NO}_{7} \mathrm{~S}$ 506.2213 Found 506.2228.

Phenyl 3-O-acetyl-4-O-benzyl-2-O-pivaloyl-6-O-triisopropylsilyl-1-thio- $\beta$-D-galactopyranoside (17a). Compound 13 (1.3 g; $2.66 \mathrm{mmol})$ was dissolved in DMF $(25 \mathrm{~mL})$ and cooled to $0{ }^{\circ} \mathrm{C}$. To the solution were added triisopropylsilyl chloride $(0.85 \mathrm{~mL} ; 3.99$ mmol) and imidazole ( $0.36 \mathrm{~g} ; 5.32 \mathrm{mmol})$. The reaction mixture was stirred at $0{ }^{\circ} \mathrm{C}$ for $1 \mathrm{~h}$ and then at $22{ }^{\circ} \mathrm{C}$ for $18 \mathrm{~h}$. The mixture was diluted with $\mathrm{Et}_{2} \mathrm{O}(200 \mathrm{~mL})$ and washed with water $(3 \times 200$ $\mathrm{mL}$ ). The organic phase was dried over $\mathrm{MgSO}_{4}$, filtered, concentrated and purified by flash chromatography (20:1 Tol/EtOAc) to give 17a as a white solid. $\mathrm{R}_{\mathrm{f}} 0.63$ (9:1 Tol/EtOAc). Yield: $1.58 \mathrm{~g}$ (93\%). IR (neat, $\mathrm{cm}^{-1}$ ): 3061.27, 3032.58, 2865.93, 1745.83, 1584.53, 1496.52, 1479.01, 1461.73, 1366.26, 1276.30, 1231.32, 1147.24, 1113.13, 1077.71, 1047.98. ${ }^{\mathbf{1}} \mathbf{H}$ NMR (400 MHz, $\left.\mathrm{CDCl}_{3}\right)$ $\delta 7.46-7.34(\mathrm{~m}, 2 \mathrm{H}, \mathrm{Ar}-\mathrm{H}), 7.35-7.07(\mathrm{~m}, 8 \mathrm{H}, \mathrm{Ar}-\mathrm{H}), 5.34\left(\mathrm{t}, J_{1,2}\right.$ $\left.=J_{2,3}=10.0 \mathrm{~Hz}, 1 \mathrm{H}, \mathrm{H}-2\right), 5.04\left(\mathrm{dd}, J_{2,3}=10.0, J_{3,4}=3.0 \mathrm{~Hz}, 1 \mathrm{H}\right.$, $\mathrm{H}-3), 4.67\left(\mathrm{~d}, J_{\mathrm{CH} 2}=11.6 \mathrm{~Hz}, 1 \mathrm{H}, 0.5 \mathrm{xCH}_{2}{ }^{\mathrm{Bn}}\right), 4.56(\mathrm{~d}, J=11.6 \mathrm{~Hz}$, $1 \mathrm{H}, 0.5 \mathrm{xCH}_{2}{ }^{\mathrm{Bn}}$ ), 3.97 (dd, $J_{3,4}=3.1, J_{4,5}=1.0 \mathrm{~Hz}, 1 \mathrm{H}, \mathrm{H}-4$ ), $3.87-$ 3.66 (m, 2H, H-6a, H-6b), 3.57 (ddd, $J_{5,6 a}=7.2, J_{5,6 b}=5.9, J_{4,5}=1.0$ $\mathrm{Hz}, 1 \mathrm{H}, \mathrm{H}-5$ ), 1.85 (s, 3H, $\mathrm{CH}_{3}{ }^{\mathrm{Ac}}$ ), 1.12 (s, 9H, 3xCH${ }_{3}^{\mathrm{Piv}}$ ), 0.98 (s, $\left.6 \mathrm{H}, \mathrm{CH}_{3}{ }^{\mathrm{TIPS}}\right) .{ }^{13} \mathrm{C}$ NMR $\left(101 \mathrm{MHz}, \mathrm{CDCl}_{3}\right) \delta 176.8,170.4,138.3$, 133.6, 132.0 (2C), 128.9 (2C), 128.4 (2C), 128.0 (2C), 127.7, 127.7, 86.9, 79.3, 75.0, 74.2, 67.8, 61.7, 38.9, 27.2 (3C), 20.9, 18.1 (2C), 18.1 (2C), 17.8, 12.4, 12.0 (2C). HRMS (ESI-TOF) m/z: $[\mathrm{M}+\mathrm{Na}]^{+}$Calcd for $\mathrm{C}_{35} \mathrm{H}_{52} \mathrm{NaO}_{7} \mathrm{SSi}$ 667.3101 Found 667.3098.

Benzyl 3-O-acetyl-4-O-benzyl-6-O-triisopropylsilyl-2-Opivaloyl- $\beta$-D-galactopyranosyl-( $1 \rightarrow 3)-4,6$-O-benzylidene-2- $O$ pivaloyl- $\beta$-D-galactopyranosyl- $(1 \rightarrow 3)-4,6-O$-benzylidene-2- $O$ pivaloyl- $\beta$-D-galactopyranoside (18a). To a $50 \mathrm{~mL}$ flame-dried flask was added 16 (0.92 g, $1.18 \mathrm{mmol})$ and 17a (1.14 g, $1.78 \mathrm{mmol})$. The mixture was dried azeotropically with toluene (2x20 mL) and subjected to vacuum overnight. It was then dissolved in dry $\mathrm{CH}_{2} \mathrm{Cl}_{2}(10 \mathrm{~mL})$ and dry $\mathrm{MeCN}(10 \mathrm{~mL})$, cooled to $-30{ }^{\circ} \mathrm{C}$, followed by addition of NIS (413 mg; $1.84 \mathrm{mmol}$ ) and TESOTf (63 mg; $0.24 \mathrm{mmol}$ ). The reaction mixture was stirred at $30{ }^{\circ} \mathrm{C}$ until TLC revealed full conversion of the donor $(1 \mathrm{~h})$. The solution was diluted with $\mathrm{CH}_{2} \mathrm{Cl}_{2}(100 \mathrm{~mL})$ and washed with sat. aq. $\mathrm{NaS}_{2} \mathrm{O}_{3}(100 \mathrm{~mL})$ and sat. aq. $\mathrm{NaHCO}_{3}(100 \mathrm{~mL})$. The organic phase was dried over $\mathrm{MgSO}_{4}$, filtered and concentrated. The product was purified by flash chromatography (12:1 toluene/EtOAc) to afford an off-white solid. Yield: $590 \mathrm{mg}$ (38\%). Rf 0.46 (9:1 Tol/EtOAc). ${ }^{1} \mathbf{H}$ NMR (400 MHz, $\left.\mathrm{CDCl}_{3}\right) \delta 7.56-7.01$ (m, 20H,

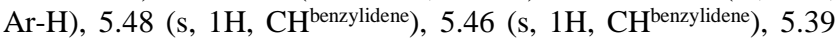
(dd, $J_{2,3}=10.3, J_{1,2}=7.8 \mathrm{~Hz}, 1 \mathrm{H}, \mathrm{H}-2$ ), 5.31 (dd, $J_{2,3}=10.1, J_{1,2}=$ $8.1 \mathrm{~Hz}, 1 \mathrm{H}, \mathrm{H}-2$ ), 5.27 (dd, $J_{2,3}=10.5, J_{1,2}=8.0 \mathrm{~Hz}, 2 \mathrm{H}$ ), 4.84 (dd, $\left.J_{2,3}=10.6, J_{3,4}=3.1 \mathrm{~Hz}, 1 \mathrm{H}, \mathrm{H}-3^{3}\right), 4.82\left(\mathrm{~d}, J_{\mathrm{CH} 2}=11.9 \mathrm{~Hz}, 1 \mathrm{H}\right.$, $\mathrm{CH}_{2}{ }^{\mathrm{Bn}}$ ), 4.71 (d, $\left.J_{1,2}=1 \mathrm{~Hz}, 1 \mathrm{H}, \mathrm{H}-1\right), 4.70$ (d, $J_{1,2}=8.0 \mathrm{~Hz}, 1 \mathrm{H}, \mathrm{H}-$ 1), $4.70\left(\mathrm{~d}, J_{\mathrm{CH} 2}=12.2 \mathrm{~Hz}, 1 \mathrm{H}, 0.5 \mathrm{xCH}_{2}{ }^{\mathrm{Bn}}\right), 4.65\left(\mathrm{~d}, J_{\mathrm{CH} 2}=11.9\right.$ $\left.\mathrm{Hz}, 1 \mathrm{H}, 0.5 \mathrm{xCH}_{2}{ }^{\mathrm{Bn}}\right), 4.55\left(\mathrm{~d}, J_{\mathrm{CH} 2}=11.3 \mathrm{~Hz}, 1 \mathrm{H}, 0.5 \mathrm{xCH}_{2}{ }^{\mathrm{Bn}}\right), 4.45$ $\left(\mathrm{d}, J_{\mathrm{CH} 2}=11.9 \mathrm{~Hz}, 1 \mathrm{H}, 0.5 \mathrm{xCH}_{2}{ }^{\mathrm{Bn}}\right.$ ), 4.37 (d, $J_{1,2}=7.8 \mathrm{~Hz}, 1 \mathrm{H}, \mathrm{H}-$ 1), 4.31 - 4.21 (m, 2H, H-6), 4.20 (d, $\left.J_{3,4}=3.5 \mathrm{~Hz}, 1 \mathrm{H}, \mathrm{H}-4\right), 4.15$ (d, $\left.J_{3,4}=3.4 \mathrm{~Hz}, 1 \mathrm{H}, \mathrm{H}-4\right), 4.12$ (d, $\left.J_{3,4}=3.4 \mathrm{~Hz}, 1 \mathrm{H}, \mathrm{H}-4\right), 4.06-$ 3.91 (m, 5H, 3xH-3, H-6), 3.81 (m, 1H, H-6a ${ }^{3}$ ), 3.71 (m, 1H, H$6 \mathrm{~b}^{3}$ ), 3.47 (dd, $\left.J_{5,6 a}=8.7, J_{5,6 b}=5.5 \mathrm{~Hz}, 1 \mathrm{H}, \mathrm{H}-5^{3}\right), 3.31(\mathrm{~s}, 1 \mathrm{H}, \mathrm{H}-$ 5), 3.27 (s, $1 \mathrm{H}, \mathrm{H}-5), 1.83$ (s, $\left.3 \mathrm{H}, \mathrm{CH}_{3}{ }^{\mathrm{Ac}}\right), 1.07$ (s, $9 \mathrm{H}, 3 \mathrm{xCH}_{3}{ }^{\mathrm{Piv}}$ ), 1.06 (s, 9H, $\left.3 \mathrm{xCH}_{3}{ }^{\mathrm{Piv}}\right), 1.03-0.96\left(\mathrm{~m}, 27 \mathrm{H}, 3 \mathrm{xCH}_{3}{ }^{\mathrm{Piv}}, 6 \mathrm{xCH}_{3}{ }^{\mathrm{TIPS}}\right)$, $0.99\left(\mathrm{~m}, 3 \mathrm{H}, 3 \times \mathrm{XCH}^{\mathrm{TIPS}}\right) .{ }^{13} \mathrm{C}$ NMR (101 MHz, $\left.\mathrm{CDCl}_{3}\right) \delta 177.2$, 176.1, 175.9, 170.3, 138.2, 137.9, 137.7, 137.3, 128.6-126.0 (20C), 100.21, 100.18, 100.1, 100.0, 99.4, 78.9, 77.7, 77.3, 75.7, 75.6, 75.3, 75.3, 74.8, 74.1, 74.0, 73.7, 73.5, 71.7, 71.4, 69.9, 69.3, 68.8, 68.7, 67.3, 67.0, 66.7, 60.9, 60.8, 38.7, 38.6, 38.6, 27.20 (3С), 27.18 (3C), 27.0 (3C), 20.7, 18.1 (3C), 18.1 (3C), 18.03 (3C), 18.02 (3C), 18.00 (3C), 17.96 (3C), 11.85, 11.85, 11.83.
Benzyl 4-O-benzyl-6-O-triisopropylsilyl-2- $O$-pivaloyl- $\beta$-D-galactopyranosyl- $(1 \rightarrow 3)-4,6$ - $O$-benzylidene-2- $O$-pivaloyl- $\beta$-D-galactopyranosyl-( $\rightarrow 3)$-4,6- $O$-benzylidene-2- $O$-pivaloyl- $\beta$-D-galactopyranoside (19a). Compound 18a (350 mg; $0.27 \mathrm{mmol}$ ) was dissolved in dry $\mathrm{CH}_{2} \mathrm{Cl}_{2}(10 \mathrm{~mL})$ and cooled to $0{ }^{\circ} \mathrm{C}$. A $1 \mathrm{M} \mathrm{L}$-selectride solution in THF $(1.1 \mathrm{~mL})$ was added and the reaction was stirred at $0{ }^{\circ} \mathrm{C}$ until complete consumption of the starting material was observed by TLC $(3 \mathrm{~h})$. The reaction mixture was poured into sat. aq. $\mathrm{NH}_{4} \mathrm{Cl}(100 \mathrm{~mL})$ and extracted with $\mathrm{CH}_{2} \mathrm{Cl}_{2}(2 \times 50 \mathrm{~mL})$. The combined organic phases were dried over $\mathrm{MgSO}_{4}$, filtered and concentrated (avoid concentrating to dryness since the borane salts can be explosive). The crude product was purified by flash chromatography (9:1 Tol/EtOAc) to give an off-white solid. Yield: 0.31 g (92\%). Rf 0.40 (9:1 Tol/EtOAc). ${ }^{1} \mathbf{H}$ NMR $\left(400 \mathrm{MHz}, \mathrm{CDCl}_{3}\right) \delta$ $7.54-7.31$ (m, 4H, Ar-H), 7.35 - 7.05 (m, 16H, Ar-H), 5.49 (s, $\left.1 \mathrm{H}, \mathrm{CH}^{\text {benzylidene }}\right), 5.43$ (s, $\left.1 \mathrm{H}, \mathrm{CH}^{\text {benzylidene }}\right), 5.40$ (dd, $J_{2,3}=10.1, J_{1,2}$ $=7.7 \mathrm{~Hz}, 1 \mathrm{H}, \mathrm{H}-2), 5.35$ (dd, $J_{2,3}=10.0, J_{1,2}=7.8 \mathrm{~Hz}, 1 \mathrm{H}, \mathrm{H}-2$ ), $4.84\left(\mathrm{dd}, J_{2,3}=9.6, J_{1,2}=7.4 \mathrm{~Hz}, 1 \mathrm{H}, \mathrm{H}-2\right), 4.83\left(\mathrm{~d}, J_{\mathrm{CH} 2}=12.1 \mathrm{~Hz}\right.$, $\left.1 \mathrm{H}, 0.5 \mathrm{xCH}_{2}{ }^{\mathrm{Bn}}\right), 4.73-4.71\left(\mathrm{~m}, 2 \mathrm{H}, \mathrm{CH}_{2}{ }^{\mathrm{Bn}}\right), 4.69\left(\mathrm{~d}, J_{1,2}=7.7 \mathrm{~Hz}\right.$, $1 \mathrm{H}, \mathrm{H}-1), 4.64$ (d, $\left.J_{1,2}=7.8 \mathrm{~Hz}, 1 \mathrm{H}, \mathrm{H}-1\right), 4.45$ (d, $J_{C H 2}=11.9 \mathrm{~Hz}$, $\left.1 \mathrm{H}, 0.5 \mathrm{xCH}_{2}{ }^{\mathrm{Bn}}\right), 4.37\left(\mathrm{~d}, J_{1,2}=7.9 \mathrm{~Hz}, 1 \mathrm{H}, \mathrm{H}-1\right), 4.31-4.20(\mathrm{~m}$, $3 \mathrm{H}, \mathrm{H}-4, \mathrm{H}-6)$ ), 4.18 - 4.12 (m, 2H, H-3, H-4), $4.02-3.93$ (m, 3H, H-3, H-6), 3.88 (dd, $J_{3,4}=3.5 \mathrm{~Hz}, J_{4,5}=1.0 \mathrm{~Hz}, 1 \mathrm{H}, \mathrm{H}-4$ ), 3.82 (dd, $\left.J_{6 a, 6 b}=9.7, J_{5,6 a}=8.6 \mathrm{~Hz}, 1 \mathrm{H}, \mathrm{H}-6 \mathrm{a}\right), 3.75$ (dd, $J_{6 a, 6 b}=9.7, J_{5,6 b}=$ $5.5 \mathrm{~Hz}, 1 \mathrm{H}, \mathrm{H}-6 \mathrm{~b}$ ), 3.46 (dd, $J_{2,3}=9.7, J_{3,4}=3.5 \mathrm{~Hz}, 1 \mathrm{H}, \mathrm{H}-3$ ), 3.37 (ddd, $J_{5,6 a}=8.6, J_{5,6 b}=5.5, J_{4,5}=1.1 \mathrm{~Hz}, 1 \mathrm{H}, \mathrm{H}-5$ ), 3.32 (d, $J=1.3$ $\mathrm{Hz}, 1 \mathrm{H}, \mathrm{H}-5$ ), 3.29 (d, $J=1.4 \mathrm{~Hz}, 1 \mathrm{H}, \mathrm{H}-5$ ), 1.08 (s, 9H, 3xCH${ }^{\mathrm{Piv}}$ ), $1.06\left(\mathrm{~s}, 9 \mathrm{H}, 3 \mathrm{xCH}_{3}{ }^{\mathrm{Piv}}\right), 1.04\left(\mathrm{~s}, 9 \mathrm{H}, 3 \mathrm{xCH}_{3}{ }^{\mathrm{Piv}}\right), 1.00(\mathrm{~s}, 18 \mathrm{H}$, $\left.6 \mathrm{xCH}_{3}{ }^{\mathrm{TIPS}}\right), 0.99$ (m, 3H, 3xCH $\left.{ }^{\mathrm{TIPS}}\right) .{ }^{13} \mathrm{C}$ NMR (101 MHz, $\left.\mathrm{CDCl}_{3}\right)$ $\delta$ 177.6, 176.10, 176.06, 137.9, 137.8, 137.34, 137.32, 127.6-125.1 (20C), 99.3, 99.2, 99.1, 98.9, 98.3, 75.2, 74.8, 74.7, 74.6, 74.3, 72.6, 72.5, 70.8, 70.74, 70.71, 70.0, 68.9, 67.72, 67.69, 66.3, 65.9, 26.2 (3C), 26.1 (3C), 25.9 (3C), 17.1 (2C), 17.05 (2C), 17.02 (2C), $10.8(3 \mathrm{C})$.

Phenyl 3-O-acetyl-6-O-allyloxycarbonyl-4-O-benzyl-2-Opivaloyl-1-thio- $\beta$-D-galactopyranoside (17c). TMEDA $(0.18 \mathrm{~mL}$; $1.22 \mathrm{mmol})$ and allyl chloroformate $(0.24 \mathrm{~mL} ; 2.25 \mathrm{mmol})$ were added to a solution of $\mathbf{1 3}$ (1.0 g; $2.05 \mathrm{mmol}$ ) in anhydrous $\mathrm{CH}_{2} \mathrm{Cl}_{2}$ $(25 \mathrm{~mL})$ at $0{ }^{\circ} \mathrm{C}$. After $1 \mathrm{~h}$ the mixture was diluted with $\mathrm{CH}_{2} \mathrm{Cl}_{2}(75$ $\mathrm{mL})$, washed with water $(100 \mathrm{~mL})$, dried over $\mathrm{MgSO}_{4}$, filtered and concentrated. The product was purified by flash chromatography (15:1 Tol/EtOAc) to give compound 17c. $\mathrm{R}_{\mathrm{f}} 0.51$ (9:1 Tol/EtOAc). Yield: 8.3 g (91\%). IR (neat, $\mathrm{cm}^{-1}$ ): 3062.34, 2972.29, 2936.14, 2905.97, 1743.53, 1584.14, 1496.611455.38, 1440.49, 1366.40, 1256.83, 1230.13, 1143.53, 1084.39. ${ }^{\mathbf{1}} \mathbf{H}$ NMR (400 MHz, $\left.\mathrm{CDCl}_{3}\right)$ $\delta 7.48$ - 7.36 (m, 2H, Ar-H), $7.34-7.09$ (m, 8H, Ar-H), 5.85 (ddt, $\left.J_{\text {trans }}=17.2, J_{\text {cis }}=10.4, J_{\mathrm{CH} 2}=5.8 \mathrm{~Hz}, 1 \mathrm{H},-\mathrm{CH}=\mathrm{CH}_{2}\right), 5.34\left(\mathrm{t}, J_{1,2}\right.$ $\left.=J_{2,3}=10.1 \mathrm{~Hz}, 1 \mathrm{H}, \mathrm{H}-2\right), 5.29\left(\mathrm{dq}, J_{\text {trans }}=17.2, J_{\mathrm{CH} 2}=1.4 \mathrm{~Hz}, 1 \mathrm{H}\right.$, $\mathrm{CH}_{2}=\mathrm{CH}_{\text {trans }}$ ), 5.21 (dd, $J_{\text {cis }}=10.4, J_{C H 2}=1.3 \mathrm{~Hz}, 1 \mathrm{H}$, $\mathrm{CH}_{2}=\mathrm{CH}_{\text {trans }}$ ), 5.00 (dd, $\left.J_{2,3}=10.0, J_{3,4}=2.9 \mathrm{~Hz}, 1 \mathrm{H}, \mathrm{H}-3\right), 4.68$ (d, $\left.J_{\mathrm{CH} 2}=11.6 \mathrm{~Hz}, 1 \mathrm{H}, \mathrm{CH}_{2}{ }^{\mathrm{Bn}}\right), 4.62\left(\mathrm{~d}, J_{1,2}=10.0 \mathrm{~Hz}, 1 \mathrm{H}, \mathrm{H}-1\right), 4.54$ (dt, $J_{\mathrm{CH}=\mathrm{CH} 2}=5.8, J_{\mathrm{CH} 2=\mathrm{CH}}=1.4 \mathrm{~Hz}, 2 \mathrm{H}, \mathrm{CH}_{2}$ allyl), $4.47\left(\mathrm{~d}, J_{\mathrm{CH} 2}=\right.$ $11.6 \mathrm{~Hz}, 1 \mathrm{H}, \mathrm{CH}_{2}{ }^{\mathrm{Bn}}$ ), 4.31 (dd, $J_{6 a, 6 b}=11.0, J_{5,6 a}=6.4 \mathrm{~Hz}, 1 \mathrm{H}, \mathrm{H}-$ $6 \mathrm{a}$ ), 4.05 (dd, $J_{6 a, 6 b}=11.0, J_{5,6 b}=6.4 \mathrm{~Hz}, 1 \mathrm{H}, \mathrm{H}-6 \mathrm{~b}$ ), 3.88 (dd, $J_{3,4}$ $\left.=3.0, J_{4,5}=1.0 \mathrm{~Hz}, 1 \mathrm{H}, \mathrm{H}-4\right), 3.74\left(\mathrm{td}, J_{5,6 b}=J_{5,6 a}=6.4, J_{4,5}=1.1\right.$ $\mathrm{Hz}, 1 \mathrm{H}, \mathrm{H}-5), 1.91$ (s, 3H, $\left.\mathrm{CH}_{3}{ }^{\mathrm{Ac}}\right), 1.14$ (s, 9H, 3xCH$\left.{ }^{\mathrm{Piv}}\right),{ }^{13} \mathrm{C}$ NMR (101 MHz, CDCl3) $\delta 176.8,170.2,154.6,137.5,133.1$, 132.5 (2C), 131.4, 129.0 (2C), 128.6 (2C), 128.2 (2C), 128.1, 128.0, 119.3, 87.1, 75.9, 75.0, 74.8, 73.9, 68.9, 67.4, 65.8, 38.9, 27.1 (3C), 20.8. HRMS (ESI-TOF) $\mathrm{m} / \mathrm{z}$ : $[\mathrm{M}+\mathrm{Na}]^{+}$Calcd for $\mathrm{C}_{30} \mathrm{H}_{36} \mathrm{NaO}_{9} \mathrm{~S} 595.1978$ Found 595.1978.

Benzyl 3-O-acetyl-4-O-benzyl-6- $O$-allyloxocarbonyl-2- $O$ pivaloyl- $\beta$-D-galactopyranosyl-( $1 \rightarrow 3)-4,6$ - $O$-benzylidene-2- $O$ pivaloyl- $\beta$-D-galactopyranosyl- $(1 \rightarrow 3)-4,6$ - $O$-benzylidene-2- $O$ pivaloyl- $\beta$-D-galactopyranoside (18c). To a $25 \mathrm{~mL}$ flame-dried 
flask was added 16 (1.0 g, $1.29 \mathrm{mmol})$ and the 17c (0.96 mg, $1.67 \mathrm{mmol}$ ). The mixture was dried azeotropically with toluene $(2 \times 10 \mathrm{~mL})$ and subjected to vacuum overnight. It was then dissolved in dry $\mathrm{CH} 2 \mathrm{Cl} 2(5 \mathrm{~mL})$ and dry MeCN (5 mL), cooled to $-30{ }^{\circ} \mathrm{C}$, followed by addition of NIS (385 mg; $1.71 \mathrm{mmol}$ ) and TESOTf (34 mg; $0.13 \mathrm{mmol}$ ). The reaction mixture was stirred at $-30{ }^{\circ} \mathrm{C}$ until TLC revealed full conversion of the donor $(2 \mathrm{~h})$. The solution was diluted with $\mathrm{CH}_{2} \mathrm{Cl}_{2}(100 \mathrm{~mL})$ and washed with sat. aq. $\mathrm{NaS}_{2} \mathrm{O}_{3}(100 \mathrm{~mL})$ and sat. aq. $\mathrm{NaHCO}_{3}(100 \mathrm{~mL})$. The organic phase was dried over $\mathrm{MgSO}_{4}$, filtered and concentrated. The product was purified by flash chromatography (9:1 toluene/EtOAc) to afford 18c as an off-white solid. Yield: $1.14 \mathrm{~g}(74 \%) .{ }^{\mathbf{1}} \mathbf{H}$ NMR $\left(400 \mathrm{MHz}, \mathrm{CDCl}_{3}\right) \delta 7.43$ (m, 4H, Ar-H), $7.39-7.05$ (m, 16H, Ar$\mathrm{H}$ ), 5.86 (ddt, $J_{\text {trans }}=17.2, J_{\text {cis }}=10.3, J_{C H 2}=5.9 \mathrm{~Hz}, 1 \mathrm{H}$, $C H=\mathrm{CH}_{2}$ ), 5.48 (s, 2H, 2xCH $\left.{ }^{\text {benzylidene }}\right), 5.38\left(\mathrm{dd}, J_{2,3}=10.1, J_{1,2}=\right.$ $7.7 \mathrm{~Hz}, 1 \mathrm{H}, \mathrm{H}-2$ ), 5.33 (dd, $J_{2,3}=10.2, J_{1,2}=7.8 \mathrm{~Hz}, 1 \mathrm{H}, \mathrm{H}-2$ ), 5.30 $\left(\mathrm{dd}, J_{\text {trans }}=17.2, J_{\mathrm{CH} 2}=1.5 \mathrm{~Hz}, 1 \mathrm{H}, \mathrm{CH}_{2}=\mathrm{CH}-\right.$ ), 5.28 (dd, $J_{2,3}=$ $\left.10.9, J_{1,2}=7.0 \mathrm{~Hz}, 1 \mathrm{H}, \mathrm{H}-2\right), 5.23$ (dq, $J_{\text {cis }}=10.4, J_{C H 2}=1.2 \mathrm{~Hz}$, $1 \mathrm{H}, \mathrm{CH}_{2}=\mathrm{CH}-$ ), 4.83 (dd, $J_{2,3}=10.9, J_{3,4}=3.6 \mathrm{~Hz}, 1 \mathrm{H}, \mathrm{H}-3$ ), 4.82 $\left(\mathrm{d}, J_{\mathrm{CH} 2}=11.7 \mathrm{~Hz}, 1 \mathrm{H}\right), 4.72\left(\mathrm{~d}, J_{1,2}=7.7 \mathrm{~Hz}, 1 \mathrm{H}, \mathrm{H}-1\right), 4.70(\mathrm{~d}$, $\left.J_{1,2}=7.0 \mathrm{~Hz}, 1 \mathrm{H}, \mathrm{H}-1\right), 4.67\left(\mathrm{~d}, J_{\mathrm{CH} 2}=11.5 \mathrm{~Hz}, 1 \mathrm{H}, \mathrm{CH}_{2}{ }^{\mathrm{Bn}}\right), 4.55$ (m, $2 \mathrm{H}, \mathrm{CH}_{2}{ }^{\mathrm{Alloc}}$ ), 4.45 (d, $J_{\mathrm{CH} 2}=12.0 \mathrm{~Hz}, 1 \mathrm{H}, \mathrm{CH}_{2}{ }^{\mathrm{Bn}}$ ), 4.44 (d, $J_{\mathrm{CH} 2}$ $\left.=11.4 \mathrm{~Hz}, 1 \mathrm{H}, 0.5 \mathrm{xCH}_{2}{ }^{\mathrm{Bn}}\right), 4.37\left(\mathrm{~d}, J_{1,2}=7.9 \mathrm{~Hz}, 1 \mathrm{H}, \mathrm{H}-1\right), 4.31-$ 4.19 (m, 3H, 1.5xH-6), 4.24 (d, $J_{3,4}=3.5 \mathrm{~Hz}, 1 \mathrm{H}, \mathrm{H}-4$ ), 4.21 (dd, $\left.J_{3,4}=3.8, J_{4,5}=1.2 \mathrm{~Hz}, 1 \mathrm{H}, \mathrm{H}-4\right), 4.14$ (dd, $J_{2,3}=9.8, J_{3,4}=3.0 \mathrm{~Hz}$, $1 \mathrm{H}, \mathrm{H}-3), 4.10$ (dd, $\left.J_{6 a, 6 b}=11.1, J_{5,6 a}=5.9 \mathrm{~Hz}, 1 \mathrm{H}, \mathrm{H}-6 \mathrm{a}\right), 4.00(\mathrm{~m}$, $2 \mathrm{H}, \mathrm{H}-6$ ), 3.95 (dd, $J_{2,3}=10.5, J_{3,4}=3.4 \mathrm{~Hz}, 1 \mathrm{H}, \mathrm{H}-3$ ), 3.86 (dd, $J_{3,4}$ $\left.=3.1, J_{4,5}=1.5 \mathrm{~Hz}, 1 \mathrm{H}, \mathrm{H}-4\right), 3.67\left(\mathrm{td}, J_{5,6 a}=5.9, J_{5,6 a}=1.5 \mathrm{~Hz}\right.$, 1H, H-5), 3.34 - 3.27 (m, 2H, 2xH-5), 1.89 (s, 3H, $\mathrm{CH}_{3}{ }^{\mathrm{Ac}}$ ), 1.09 (s, $8 \mathrm{H}, 3 \mathrm{xCH}_{3}{ }^{\text {Piv }}$ ), 1.05 (s, 9H, 3xCH${ }_{3}{ }^{\text {Piv }}$ ), 1.01 (s, 9H, $\left.3 \mathrm{xCH}_{3}{ }^{\text {Piv }}\right) .{ }^{13} \mathrm{C}$ NMR (101 MHz, $\left.\mathrm{CDCl}_{3}\right) \delta 177.1,176.2,176.1,170.3,154.7$, 138.0, 137.8, 137.38, 137.37, 131.4-126.1 (20C), 119.5, 100.4, 100.3, 100.2, 100.0, 99.9, 75.81, 75.76, 75.1, 73.8, 73.2, 72.8, 72.7, 71.9, 71.8, 70.9, 70.0, 68.9, 68.8, 67.4, 67.1, 66.0, 38.9, 38.8, 38.7, 27.3 (6C), 27.1 (3C), 20.8. HRMS (ESI-TOF) m/z: [M + Na ${ }^{+}$ Calcd for $\mathrm{C}_{67} \mathrm{H}_{82} \mathrm{NaO}_{22}$ : 1261.5195; Found 1261.5197 .

Benzyl 3-O-acetyl-4-O-benzyl-2-O-pivaloyl-6-O-(tert-butyldiphenylsilyl)- $\beta$-D-galactopyranosyl-( $1 \rightarrow 3)-4,6$ - $O$-benzylidene-2$O$-pivaloyl- $\beta$-D-galactopyranosyl- $(1 \rightarrow 3)-4,6$ - $O$-benzylidene-2-

$\boldsymbol{O}$-pivaloyl- $\boldsymbol{\beta}$-D-galactopyranoside (18b). To a $25 \mathrm{~mL}$ flame-dried flask was added 16 (800 mg, $1.03 \mathrm{mmol})$ and the 17b (1050 mg, $1.45 \mathrm{mmol}$ ). The mixture was dried azeotropically with toluene (2x10 $\mathrm{mL})$ and subjected to vacuum overnight. It was then dissolved in dry $\mathrm{CH}_{2} \mathrm{Cl}_{2}(5 \mathrm{~mL})$ and dry $\mathrm{MeCN}(5 \mathrm{~mL})$, cooled to $-30{ }^{\circ} \mathrm{C}$, followed by addition of NIS (333 $\mathrm{mg} ; 1.48 \mathrm{mmol}$ ) and TESOTf (54 mg; $0.21 \mathrm{mmol}$ ). The reaction mixture was stirred at $-30{ }^{\circ} \mathrm{C}$ until TLC revealed full conversion of the donor $(2 \mathrm{~h})$. The solution was diluted with $\mathrm{CH}_{2} \mathrm{Cl}_{2}(100 \mathrm{~mL})$ and washed with sat. aq. $\mathrm{NaS}_{2} \mathrm{O}_{3}(100 \mathrm{~mL})$ and sat. aq. $\mathrm{NaHCO}_{3}(100 \mathrm{~mL})$. The organic phase was dried over $\mathrm{MgSO}_{4}$, filtered and concentrated. The product was purified by flash chromatography (9:1 toluene/EtOAc) to afford an off-white solid. Yield: $1.02 \mathrm{~g}$ (71\%). ${ }^{\mathbf{1}} \mathbf{H}$ NMR (400 MHz, $\left.\mathrm{CDCl}_{3}\right) \delta 7.55$ (m, 4H, Ar-H), $7.51-7.04$ (m, 32H, Ar-H), 5.47 (s, $\left.1 \mathrm{H}, \mathrm{H}^{\text {benzylidene }}\right), 5.42\left(\mathrm{~s}, 1 \mathrm{H}, \mathrm{H}^{\text {benzylidene }}\right), 5.34$ (dd, $J_{2,3}=10.3, J_{1,2}=$ $7.9 \mathrm{~Hz}, 1 \mathrm{H}$ ), 5.26 (dd, $J_{2,3}=10.7, J_{1,2}=7.9 \mathrm{~Hz}, 1 \mathrm{H}, \mathrm{H}-1$ ), 5.25 (dd, $\left.J_{2,3}=10.6, J_{1,2}=7.9 \mathrm{~Hz}, 1 \mathrm{H}, \mathrm{H}-1\right), 4.85$ (dd, $J_{2,3}=10.6, J_{3,4}=3.1$ $\mathrm{Hz}, 1 \mathrm{H}, \mathrm{H}-3), 4.81\left(\mathrm{~d}, J_{\mathrm{CH} 2}=11.9 \mathrm{~Hz}, 1 \mathrm{H}, 0.5 \mathrm{xCH}_{2}{ }^{\mathrm{Bn}}\right), 4.68(\mathrm{~d}$, $\left.J_{\mathrm{CH} 2}=11.3 \mathrm{~Hz}, 1 \mathrm{H}, 0.5 \mathrm{xCH}_{2}{ }^{\mathrm{Bn}}\right), 4.64\left(\mathrm{~d}, J_{1,2}=7.9 \mathrm{~Hz}, 1 \mathrm{H}, \mathrm{H}-1\right)$, $4.59\left(\mathrm{~d}, J_{1,2}=7.9 \mathrm{~Hz}, 1 \mathrm{H}, \mathrm{H}-1\right), 4.54\left(\mathrm{~d}, J_{\mathrm{CH} 2}=11.3 \mathrm{~Hz}, 1 \mathrm{H}\right.$, $\left.0.5 \mathrm{xCH}_{2}{ }^{\mathrm{Bn}}\right), 4.44\left(\mathrm{~d}, J_{\mathrm{CH} 2}=11.9 \mathrm{~Hz}, 1 \mathrm{H}, 0.5 \mathrm{xCH}_{2}{ }^{\mathrm{Bn}}\right), 4.35\left(\mathrm{~d}, J_{1,2}\right.$ $=7.9 \mathrm{~Hz}, 1 \mathrm{H}, \mathrm{H}-1), 4.28-4.18(\mathrm{~m}, 3 \mathrm{H}, \mathrm{H}-4, \mathrm{H}-6), 4.13$ (d, $J_{3,4}=$ $3.6 \mathrm{~Hz}, 1 \mathrm{H}, \mathrm{H}-4$ ), 4.10 (dd, $J_{2,3}=10.3, J_{3,4}=3.4 \mathrm{~Hz}, 1 \mathrm{H}, \mathrm{H}-3$ ), 4.05 (d, $\left.J_{3,4}=3.7 \mathrm{~Hz}, 1 \mathrm{H}, \mathrm{H}-4\right), 3.95(\mathrm{~m}, 1 \mathrm{H}, \mathrm{H}-6), 3.90$ (dd, $J_{2,3}=10.3$, $\left.J_{3,4}=3.4 \mathrm{~Hz}, 1 \mathrm{H}, \mathrm{H}-3\right), 3.83\left(\mathrm{t}, J_{6 \mathrm{a}, 6 \mathrm{~b}}=J_{5,6 \mathrm{a}}=9.8 \mathrm{~Hz}, 1 \mathrm{H}, \mathrm{H}-6 \mathrm{a}\right)$, 3.66 (dd, $\left.J_{6 \mathrm{a}, 6 \mathrm{~b}}=9.8, J_{5,6 \mathrm{~b}}=5.4 \mathrm{~Hz}, 1 \mathrm{H}, \mathrm{H}-6 \mathrm{~b}\right), 3.53-3.44(\mathrm{~m}, 1 \mathrm{H}$, H-5), 3.29 (m, 1H, H-5), 3.19 (m, 1H, H-5), 1.86 (s, 3H, CH3 ${ }^{\mathrm{Ac}}$ ),
1.00 (s, 9H, $\left.3 \mathrm{xCH}_{3}{ }^{\mathrm{Piv}}\right), 1.00$ (s, $\left.12 \mathrm{H}, 3 \mathrm{xCH}_{3}{ }^{\mathrm{Piv}}\right), 0.98$ (s, 9H, $\left.3 \mathrm{xCH}_{3}{ }^{\mathrm{Piv}}\right), 0.91\left(\mathrm{~s}, 9 \mathrm{H}, 3 \mathrm{xCH}_{3}{ }^{\mathrm{TBDPS}}\right) .{ }^{13} \mathbf{C}$ NMR $\left(101 \mathrm{MHz}^{\mathrm{C}} \mathrm{CDCl}_{3}\right)$ $\delta$ 177.3, 176.2, 176.1, 170.4, 138.3, 138.0, 137.8, 137.4, 135.62, 135.55, 133.0-126.2 (30C), 100.4, 100.2, 100.2, 100.0, 99.5, 77.4, 75.8, 75.7, 75.4, 74.8, 74.4, 73.9, 71.80, 71.77, 71.65, 70.8, 70.0, 69.4, 68.9, 68.8, 67.4, 67.1, 61.4, 38.8, 38.7, 38.6, 27.3 (3C), 27.2 (3C), 27.13 (3C), 27.05 (3C), 20.9, 19.3. HRMS (ESI-TOF) m/z: $[\mathrm{M}+\mathrm{Na}]^{+}$Calcd for $\mathrm{C}_{79} \mathrm{H}_{96} \mathrm{NaO}_{20} \mathrm{Si}$ : 1415.6162; Found 1415.6163.

Benzyl 4-O-benzyl-2-O-pivaloyl-6-O-(tert-butyldiphenylsilyl)$\beta$-D-galactopyranosyl-(1 $\rightarrow 3)-4,6$ - $O$-benzylidene-2- $O$-pivaloyl$\beta$-D-galactopyranosyl-(1 $\rightarrow 3)-4,6-O$-benzylidene-2- $O$-pivaloylק-D-galactopyranoside (19b). Compound 18b (1.0 g; $0.72 \mathrm{mmol})$ was dissolved in dry $\mathrm{CH}_{2} \mathrm{Cl}_{2}(25 \mathrm{~mL})$ and cooled to $0^{\circ} \mathrm{C}$. A $1 \mathrm{M} \mathrm{L}$ selectride solution in THF $(1.5 \mathrm{~mL})$ was added and the reaction was stirred at $0{ }^{\circ} \mathrm{C}$ until complete consumption of the starting material $(4 \mathrm{~h})$. The reaction mixture was poured into sat. aq. $\mathrm{NH}_{4} \mathrm{Cl}$ $(100 \mathrm{~mL})$. The organic phase was dried over $\mathrm{MgSO}_{4}$, filtered and concentrated (avoid concentrating to dryness since the borane salts can be explosive). The crude product was purified by flash chromatography to give an off-white solid. Yield: $0.84 \mathrm{~g}(88 \%)$. ${ }^{1} \mathbf{H}$ NMR $\left(400 \mathrm{MHz}, \mathrm{CDCl}_{3}\right) \delta 7.57$ (m, 4H, Ar-H), $7.49-7.07$ (m, $32 \mathrm{H}, \mathrm{Ar}-\mathrm{H}$ ), 5.48 (s, $1 \mathrm{H}, \mathrm{H}^{\text {benzylidene }}$ ), 5.43 (dd, $\mathrm{J}_{2,3}=10.4, J_{1,2}=7.6$ $\mathrm{Hz}, 1 \mathrm{H}) 5.40$ (s, $\left.1 \mathrm{H}, \mathrm{H}^{\text {benzylidene }}\right), 5.26$ (dd, $J_{2,3}=10.2, J_{1,2}=7.8 \mathrm{~Hz}$, $1 \mathrm{H}, \mathrm{H}-1), 4.83\left(\mathrm{~d}, J_{\mathrm{CH} 2}=11.8 \mathrm{~Hz}, 1 \mathrm{H}, 0.5 \mathrm{XCH}_{2}{ }^{\mathrm{Bn}}\right), 4.80-4.72(\mathrm{~m}$, $1 \mathrm{H}, \mathrm{H}-2), 4.75$ (d, $\left.J_{1,2}=7.6 \mathrm{~Hz}, 1 \mathrm{H}, \mathrm{H}-1\right), 4.67$ (d, $J_{C H 2}=11.4 \mathrm{~Hz}$, $\left.1 \mathrm{H}, 0.5 \mathrm{xCH}_{2}{ }^{\mathrm{Bn}}\right), 4.47\left(\mathrm{~d}, J_{\mathrm{CH} 2}=11.8 \mathrm{~Hz}, 1 \mathrm{H}, 0.5 \mathrm{xCH}_{2}{ }^{\mathrm{Bn}}\right), 4.38(\mathrm{~d}$, $\left.J_{1,2}=8.0 \mathrm{~Hz}, 1 \mathrm{H}, \mathrm{H}-1\right), 4.36\left(\mathrm{~d}, J_{\mathrm{CH} 2}=11.4 \mathrm{~Hz}, 1 \mathrm{H}, 0.5 \mathrm{xCH}_{2} \mathrm{Bn}^{\mathrm{Bn}}\right.$, 4.25 (d, $\left.J_{3,4}=3.2 \mathrm{~Hz}, 1 \mathrm{H}, \mathrm{H}-4\right), 4.21$ (d, $\left.J_{6 a, 6 b}=11.5 \mathrm{~Hz}, 1 \mathrm{H}, \mathrm{H}-6 \mathrm{a}\right)$, $4.31-4.10$ (m, 4H, H-1 $\left.{ }^{1}, 2 x H-4, H-6 b\right), 3.98$ (d, $J_{6 a, 6 b}=12.4 \mathrm{~Hz}$, 1H, H-6a), 3.89 - 3.78 (m, 4H, H-3, H-5, H-6b), 3.72 (m, 1H, H6a), 3.66 - 3.59 (m, 1H, H-6b), 3.56 - 3.43 (m, 2H, 2xH-3), 3.30 (s, 1H, H-5), 3.18 (s, 1H, H-5), 1.15 (s, 9H, 3xCH ${ }^{\mathrm{Piv}}$ ), 1.09 (s, 9H, $\left.3 \mathrm{xCH}_{3}{ }^{\mathrm{Piv}}\right), 1.06\left(\mathrm{~s}, 9 \mathrm{H}, 3 \mathrm{xCH}_{3}{ }^{\mathrm{Piv}}\right), 0.97\left(\mathrm{~s}, 9 \mathrm{H}, 3 \mathrm{xCH}_{3}{ }^{\mathrm{TBDPS}}\right) .{ }^{13} \mathrm{C}$ NMR $\left(101 \mathrm{MHz}, \mathrm{CDCl}_{3}\right) \delta 178.4,178.3,176.2,138.1,137.9$, 137.8, 137.4, 135.7 (2C), 135.6 (2C), 133.4, 133.1, 130.0-126.4 (26C), 105.7, 100.9, 100.5, 100.3, 99.6, 78.4, 76.2, 75.9, 75.4, 75.2 (2C), 74.5, 72.3, 71.3, 70.5, 69.9, 69.4, 68.9, 68.8, 67.0 (2C), 62.5, 39.2, 39.1, 38.8, 27.4 (3C), 27.3 (3C), 27.1 (3C), 27.0 (3C), 19.3. HRMS (ESI-TOF) m/z: $[\mathrm{M}+\mathrm{Na}]^{+}$Calcd for $\mathrm{C}_{77} \mathrm{H}_{94} \mathrm{NaO}_{19} \mathrm{Si}$ : 1373.6056; Found 1373.6061.

Phenyl 3-O-acetyl-6-O-allyl-4-O-benzyl-2-O-pivaloyl-1-thio- $\beta$ D-galactopyranoside (17d). To a solution of $\mathrm{Pd}_{2}(\mathrm{dba})_{3}$ (328 mg; $0.36 \mathrm{mmol}$ ) and 1,4-Bis(diphenyl-phosphino)butane (611 mg; $1.43 \mathrm{mmol})$ in dry THF (20 mL) was added the alcohol 13 (1.75 g; $3.6 \mathrm{mmol}$ ) and allyl ethyl carbonate (1.86 g; $14.32 \mathrm{mmol})$ in dry THF $(20 \mathrm{~mL})$. The solution was stirred at $65{ }^{\circ} \mathrm{C}$ for $4 \mathrm{~h}$, the solvent was evaporated and the crude product was purified by flash chromatographed (30:1 Tol/EtOAc) to give the pure $O$-allylated compound 17d. $\mathrm{R}_{\mathrm{f}} 0.60$ (9:1 Tol/EtOAc). Yield: $1.56 \mathrm{~g}$ (83\%). IR (neat, $\left.\mathrm{cm}^{-1}\right)$ : 3061.43, 3031.49, 2973.33, 2933.85, 2907.2, 2871.32, 1744.39, 1496.49, 1479.33, 1456.24, 1397.69, 1366.21, 1276.47, 1232.00, 1145.02, $1078.08,1047.78,918.76{ }^{1} \mathbf{H}$ NMR $(400 \mathrm{MHz}$, $\left.\mathrm{CDCl}_{3}\right) \delta 7.46-7.35$ (m, 2H, Ar-H), 7.35 - $7.13(\mathrm{~m}, 8 \mathrm{H}, \mathrm{Ar}-\mathrm{H})$, 5.78 (ddt, $J_{\text {trans }}=17.2, J_{\text {cis }}=10.8, J_{C H 2}=5.6 \mathrm{~Hz}, 1 \mathrm{H},-\mathrm{CH}=\mathrm{CH} 2$ ), $5.34\left(\mathrm{t}, J_{1,2}=J_{2,3}=10.0 \mathrm{~Hz}, 1 \mathrm{H}, \mathrm{H}-2\right), 5.17$ (dd, $J_{\text {trans }}=17.2, J_{C H 2}=$ $\left.1.7 \mathrm{~Hz}, 1 \mathrm{H}, \mathrm{CH}_{2}=\mathrm{CH}_{\text {trans }}\right), 5.10\left(\mathrm{dt}, J_{\text {cis }}=10.4, J_{\mathrm{CH} 2}=1.7 \mathrm{~Hz}, 1 \mathrm{H}\right.$, $\mathrm{CH}_{2}=\mathrm{CH}_{\text {cis }}$ ), 5.00 (dd, $J_{2,3}=10.0, J_{3,4}=3.0 \mathrm{~Hz}, 1 \mathrm{H}, \mathrm{H}-3$ ), 4.65 (d, $\left.J=11.6 \mathrm{~Hz}, 1 \mathrm{H}, 0.5 \mathrm{xCH}_{2}{ }^{\mathrm{Bn}}\right), 4.63\left(\mathrm{~d}, J_{1,2}=10.0 \mathrm{~Hz}, 1 \mathrm{H}, \mathrm{H}-1\right), 4.50$ $\left(\mathrm{d}, J_{C H 2}=11.6 \mathrm{~Hz}, 1 \mathrm{H}, 0.5 \mathrm{xCH}_{2}{ }^{\mathrm{Bn}}\right), 3.93\left(\mathrm{dd}, J_{3,4}=3.1, J_{4,5}=0.9\right.$ $\mathrm{Hz}, 1 \mathrm{H}, \mathrm{H}-4$ ), 3.89 (ddt, $J_{\mathrm{CH} 2 \mathrm{a}, \mathrm{CH} 2 \mathrm{~b}}=12.8, J_{\mathrm{CH}=\mathrm{CH} 2, \mathrm{CH} 2}=5.6, J_{\mathrm{CH} 2=\mathrm{CH}}$, $\left.\mathrm{CH}_{2}=1.7 \mathrm{~Hz}, 1 \mathrm{H}, \mathrm{CH}_{2}=\mathrm{CH}\right), 3.82\left(\mathrm{ddt}, J_{\mathrm{CH} 2 \mathrm{a}, \mathrm{CH} 2 \mathrm{~b}}=12.7, J_{\mathrm{CH}=\mathrm{CH} 2}\right.$, $\mathrm{CH}_{2}=5.6, J_{\mathrm{CH} 2}=\mathrm{CH}, \mathrm{CH} 2=1.7 \mathrm{~Hz}, 1 \mathrm{H}, \mathrm{CH}_{2}=\mathrm{CH}$ ), 3.68 (ddd, $J_{5,6 a}=6.9$, $J_{5,6 b}=5.8, J_{4,5}=1.0 \mathrm{~Hz}, 1 \mathrm{H}, \mathrm{H}-5$ ), $3.60-3.46$ (m, 1H, H-6a, H-6b), 1.87 (s, 3H, $\left.\mathrm{CH}_{3}{ }^{\mathrm{Ac}}\right), 1.13$ (s, 9H, 3xCH$\left.{ }_{3}^{\mathrm{Piv}}\right) .{ }^{13} \mathrm{C}$ NMR $(101 \mathrm{MHz}$, $\left.\mathrm{CDCl}_{3}\right) \delta 176.8,170.3,138.1,134.4,133.6,132.2(2 \mathrm{C}), 129.0(2 \mathrm{C})$, 
128.5 (2C), 128.2 (2C), 127.9, 127.8, 117.4, 87.1, 77.4, 75.0, 74.9, 74.4, 72.5, 68.2, 67.7, 38.9, 27.2 (3C), 20.9. HRMS (ESI-TOF) $\mathrm{m} / \mathrm{z}:[\mathrm{M}+\mathrm{Na}]^{+}$Calcd for $\mathrm{C}_{29} \mathrm{H}_{36} \mathrm{NaO}_{7} \mathrm{~S}$ : 551.2079; Found 551.2082 .

Benzyl 3-O-acetyl-6-O-allyl-4-O-benzyl-2-O-pivaloyl- $\beta$-D-galactopyranosyl-( $1 \rightarrow 3)$-4,6-O-benzylidene-2- $O$-pivaloyl- $\beta$-D-galactopyranosyl-( $\rightarrow 3)$-4,6-O-benzylidene-2-O-pivaloyl- $\beta$-D-galactopyranoside (18d). To a $25 \mathrm{~mL}$ flame-dried flask was added 16 (900 mg, $1.16 \mathrm{mmol}$ ) and 17d (918 mg, $1.74 \mathrm{mmol}$ ). The mixture was dried azeotropically with toluene $(2 \times 10 \mathrm{~mL})$ and subjected to vacuum overnight. It was then dissolved in dry $\mathrm{CH}_{2} \mathrm{Cl}_{2}(5 \mathrm{~mL})$ and dry MeCN (5 mL), cooled to $-30^{\circ} \mathrm{C}$, followed by addition of NIS (442 mg; $1.97 \mathrm{mmol}$ ) and TESOTf (61 mg; $0.23 \mathrm{mmol}$ ). The reaction mixture was stirred at $-30{ }^{\circ} \mathrm{C}$ until TLC revealed full conversion of the donor $(2 \mathrm{~h})$. The solution was diluted with $\mathrm{CH}_{2} \mathrm{Cl}_{2}$ $(100 \mathrm{~mL})$ and washed with sat. aq. $\mathrm{NaS}_{2} \mathrm{O}_{3}(100 \mathrm{~mL})$ and sat. aq. $\mathrm{NaHCO}_{3}(100 \mathrm{~mL})$. The organic phase was dried over MgSO4, filtered and concentrated. The product was purified by flash chromatography to afford as a slightly yellow solid. Yield: $707 \mathrm{mg}$ (51\%). ${ }^{1} \mathbf{H}$ NMR (400 MHz, CDCl $) \delta 7.57-7.46$ (m, 4H, Ar-H), $7.39-$ $7.22(\mathrm{~m}, 16 \mathrm{H}, \mathrm{Ar}-\mathrm{H}), 5.83$ (ddt, $J_{\text {trans }}=17.2, J_{\text {cis }}=10.3, J_{\mathrm{CH} 2}=5.9$ $\left.\mathrm{Hz}, 1 \mathrm{H},-\mathrm{CH}=\mathrm{CH}_{2}\right), 5.55$ (s, $\left.1 \mathrm{H}, \mathrm{CH}^{\text {benzylidene }}\right), 5.54$ (s, $1 \mathrm{H}, \mathrm{CH}^{\text {ben- }}$ zylidene), 5.45 (dd, $J_{2,3}=10.0, J_{1,2}=7.9 \mathrm{~Hz}, 1 \mathrm{H}, \mathrm{H}-2$ ), 5.41 (dd, $J_{2,3}=$ 10.3, $\left.J_{1,2}=8.0 \mathrm{~Hz}, 1 \mathrm{H}, \mathrm{H}-2\right), 5.36\left(\mathrm{dd}, J_{2,3}=10.1, J_{1,2}=7.9 \mathrm{~Hz}, 1 \mathrm{H}\right.$, $\mathrm{H}-2), 5.23\left(\mathrm{dq}, J_{\text {trans }}=17.2, J_{\mathrm{CH} 2}=1.5 \mathrm{~Hz}, 1 \mathrm{H}, \mathrm{CH}_{2}=\mathrm{CH}-\right), 5.17$ (dd, $J_{\text {cis }}=10.3, J_{\mathrm{CH} 2}=1.5 \mathrm{~Hz}, 1 \mathrm{H}, \mathrm{CH}_{2}=\mathrm{CH}-$ ), 4.90 (d, $J_{\mathrm{CH} 2}=12.0$ $\mathrm{Hz}, 1 \mathrm{H}, \mathrm{CH}_{2}{ }^{\mathrm{Bn}}$ ), 4.86 (dd, $J_{2,3}=10.1, J_{3,4}=3.1 \mathrm{~Hz}, 1 \mathrm{H}, \mathrm{H}-3$ ), 4.80 $\left(\mathrm{d}, J_{\mathrm{CH} 2}=11.3 \mathrm{~Hz}, 1 \mathrm{H}, \mathrm{CH}_{2}{ }^{\mathrm{Bn}}\right), 4.77\left(\mathrm{~d}, J_{1,2}=7.9 \mathrm{~Hz}, 1 \mathrm{H}, \mathrm{H}-1\right)$, $4.63\left(\mathrm{~d}, J_{1,2}=8.0 \mathrm{~Hz}, 1 \mathrm{H}, \mathrm{H}-1\right), 4.54$ (d, $J_{\mathrm{CH} 2}=11.9 \mathrm{~Hz}, 1 \mathrm{H}, \mathrm{CH}_{2}{ }^{\mathrm{Bn}}$ ), $4.45\left(\mathrm{~d}, J_{1,2}=7.9 \mathrm{~Hz}, 1 \mathrm{H}, \mathrm{H}-1\right), 4.44\left(\mathrm{~d}, J_{\mathrm{CH} 2}=11.3 \mathrm{~Hz}, 1 \mathrm{H}, \mathrm{CH}_{2}{ }^{\mathrm{Bn}}\right.$ ), $4.36-4.29$ (m, 3H, H-4, H-6), 4.25 (d, $\left.J_{3,4}=3.3 \mathrm{~Hz}, 1 \mathrm{H}, \mathrm{H}-4\right), 4.24$ (dd, $J_{2,3}=10.1, J_{3,4}=3.3 \mathrm{~Hz}, 1 \mathrm{H}, \mathrm{H}-3$ ), $4.08-4.02$ (m, 2H, H-6), 3.97 (d, $\left.J_{3,4}=3.1 \mathrm{~Hz}, 1 \mathrm{H}, \mathrm{H}-4\right), 3.92$ (dd, $J_{5,6 a}=4.9, J_{5,6 b}=1.3 \mathrm{~Hz}$, $1 \mathrm{H}, \mathrm{H}-5), 3.88$ (tt, $J_{C H}=5.9, J_{C H 2}=1.5 \mathrm{~Hz}, 2 \mathrm{H}, \mathrm{CH}_{2}{ }^{\mathrm{Allyl}}$ ), 3.81 (dd, $\left.J_{2,3}=10.1, J_{3,4}=3.3 \mathrm{~Hz}, 1 \mathrm{H}, \mathrm{H}-3\right), 3.65$ (t, $\left.J=6.5 \mathrm{~Hz}, 1 \mathrm{H}, \mathrm{H}-5\right)$, 3.56 - 3.44 (m, 2H, H-6), 3.39 (s, 1H, H-5), 3.35 (s, 1H, H-5), 1.96 (s, 3H, $\mathrm{CH}_{3}{ }^{\mathrm{Ac}}$ ), 1.17 (s, 9H, 3xCH $3{ }^{\mathrm{Piv}}$ ), 1.15 (s, 9H, 3xCH${ }_{3}{ }^{\mathrm{Piv}}$ ), 1.13 (s, 9H, $3 \mathrm{xCH}_{3}{ }^{\text {Piv }}$ ). HRMS (ESI-TOF) $\mathrm{m} / \mathrm{z}$ : $[\mathrm{M}+\mathrm{Na}]^{+}$Calcd for $\mathrm{C}_{64} \mathrm{H}_{80} \mathrm{NaO}_{19}$ 1175.5294; Found 1175.5154.

Benzyl 6-O-allyl-4-O-benzyl-2-O-pivaloyl- $\beta$-D-galactopyranosyl-( $1 \rightarrow 3)-4,6$ - $O$-benzylidene-2- $O$-pivaloyl- $\beta$-D-galactopyranosyl-( $1 \rightarrow 3)$-4,6- $O$-benzylidene-2- $O$-pivaloyl- $\beta$-D-galactopyranoside (19d). Compound 18d (200 mg; $0.17 \mathrm{mmol}$ ) was dissolved in dry $\mathrm{CH}_{2} \mathrm{Cl}_{2}(10 \mathrm{~mL})$ and cooled to $0{ }^{\circ} \mathrm{C}$. A $1 \mathrm{M} \mathrm{L}$-selectride solution in THF $(0.7 \mathrm{~mL})$ was added and the reaction was stirred at 0 ${ }^{\circ} \mathrm{C}$ until complete consumption of the starting material $(3 \mathrm{~h})$. The reaction mixture was poured into sat. aq. $\mathrm{NH}_{4} \mathrm{Cl}(100 \mathrm{~mL})$. The organic phase was dried over $\mathrm{MgSO}_{4}$, filtered and concentrated (avoid concentrating to dryness since the borane salts can be explosive). The crude product was purified by flash chromatography to give a foamy white product. Yield: $191 \mathrm{mg}(92 \%) .{ }^{1} \mathbf{H}$ NMR (400 $\left.\mathrm{MHz} \mathrm{CDCl}_{3}\right) \delta 7.59-7.45(\mathrm{~m}, 4 \mathrm{H}, \mathrm{Ar}-\mathrm{H}), 7.41-7.19(\mathrm{~m}, 16 \mathrm{H}$, Ar-H), 5.85 (ddt, $J_{\text {trans }}=17.2, J_{\text {cis }}=10.3, J_{C H 2}=5.9 \mathrm{~Hz}, 1 \mathrm{H}$, $\left.\mathrm{CH}=\mathrm{CH}_{2}\right), 5.57$ (s, $\left.1 \mathrm{H}, \mathrm{CH}^{\text {benzylidene }}\right), 5.52$ (s, $\left.1 \mathrm{H}, \mathrm{CH}^{\text {benzylidene }}\right), 5.52$ (dd, $\left.J_{2,3}=10.1, J_{1,2}=7.7 \mathrm{~Hz}, 1 \mathrm{H}, \mathrm{H}-2\right), 5.35$ (dd, $J_{2,3}=10.2, J_{1,2}=$ $8.0 \mathrm{~Hz}, 1 \mathrm{H}, \mathrm{H}-2), 5.23\left(\mathrm{dq}, J_{\text {trans }}=17.1, J_{\mathrm{CH} 2}=1.6 \mathrm{~Hz}, 1 \mathrm{H}\right.$, $\mathrm{CH}_{2}=\mathrm{CH}-$ ), 5.17 (dd, $J_{\text {cis }}=10.3, J_{\mathrm{CH} 2}=1.4 \mathrm{~Hz}, 1 \mathrm{H}, \mathrm{CH}_{2}=\mathrm{CH}-$ ), 4.91 $\left(\mathrm{d}, J_{\mathrm{CH} 2}=11.9 \mathrm{~Hz}, 1 \mathrm{H}, \mathrm{CH}_{2}{ }^{\mathrm{Bn}}\right), 4.87\left(\mathrm{~d}, J_{1,2}=8.1 \mathrm{~Hz}, 1 \mathrm{H}, \mathrm{H}-1\right)$, 4.82 (dd, $J_{2,3}=10.3, J_{3,4}=3.1 \mathrm{~Hz}, 1 \mathrm{H}, \mathrm{H}-3$ ), 4.77 (d, $J_{C H 2}=11.4$ $\left.\mathrm{Hz}, 1 \mathrm{H}, \mathrm{CH}_{2}{ }^{\mathrm{Bn}}\right), 4.56\left(\mathrm{~d}, J_{\mathrm{CH} 2}=11.9 \mathrm{~Hz}, 1 \mathrm{H}, \mathrm{CH}_{2}{ }^{\mathrm{Bn}}\right), 4.47\left(\mathrm{~d}, J_{1,2}=\right.$ $7.6 \mathrm{~Hz}, 1 \mathrm{H}, \mathrm{H}-1$ ), 4.44 (d, $J_{\mathrm{CH} 2}=11.3 \mathrm{~Hz}, 1 \mathrm{H}, \mathrm{CH}_{2}{ }^{\mathrm{Bn}}$ ), $4.36-4.26$ (m, 4H, H-2, H-4, H-4, H-6), 4.27 (d, $J_{1,2}=7.7 \mathrm{~Hz}, 1 \mathrm{H}, \mathrm{H}-1$ ), 4.24 (dd, $\left.J_{2,3}=10.3, J_{3,4}=3.4 \mathrm{~Hz}, 1 \mathrm{H}, \mathrm{H}-3\right), 4.12-4.00$ (m, 2H, H-4, H6), $3.95-3.85$ (m, 3H, H-3, $\left.\mathrm{CH}_{2}{ }^{\text {Allyl }}\right) 3.71-3.42$ (m, 3H, H-5, H6), 3.40 (s, $1 \mathrm{H}, \mathrm{H}-5), 3.38$ (s, $1 \mathrm{H}, \mathrm{H}-5), 1.21$ (s, 9H, $3 \mathrm{xCH}_{3}{ }^{\mathrm{Piv}}$ ), 1.18 (s, 9H, $\left.3 \mathrm{xCH}_{3}{ }^{\mathrm{Piv}}\right), 1.12$ (s, 9H, $\left.3 \mathrm{xCH}_{3}{ }^{\mathrm{Piv}}\right) .{ }^{13} \mathrm{C}$ NMR $(101 \mathrm{MHz}$, $\left.\mathrm{CDCl}_{3}\right) \delta 178.27,176.23,138.13,137.92,137.81,137.37,134.55$, 129.07, 128.76, 128.49, 128.41, 128.32, 128.27, 128.10, 128.04, 127.99, 127.95, 127.64, 126.49, 126.36, 117.25, 105.45, 101.00, 100.53, 100.39, 99.56, 78.22, 77.36, 76.21, 76.01, 75.17, 74.62, $73.90,72.44,72.34,71.17,70.60,69.95,69.29,68.94,68.87$, 68.83, 67.11, 67.04, 39.17, 39.09, 38.84, 27.38, 27.29, 27.09. HRMS (ESI-TOF) m/z: $[\mathrm{M}+\mathrm{Na}]^{+}$Calcd for $\mathrm{C}_{66} \mathrm{H}_{82} \mathrm{NaO}_{20}$ 1217.5292; Found 1217.5260.

Phenyl 4,6- $O$-benzylidene-3- $O$-chloroacetyl-1-thio- $\beta$-D-galactopyranoside (9b) ${ }^{45}$. Di-n-butyl tin oxide (21.8 g; $87.4 \mathrm{mmol}$ ) was added to a solution of compound 8 (30.0 g, $83.2 \mathrm{mmol})$ in dry toluene $(600 \mathrm{~mL})$ and stirred under refluxing temperature for $12 \mathrm{~h}$. The reaction mixture was cooled to $0{ }^{\circ} \mathrm{C}$, and freshly activated $4 \AA$ MS (30 g) were added. After 30 min chloroacetyl chloride $(6.7 \mathrm{~mL}$, $85.7 \mathrm{mmol}$ ) were added drop wise and stirring was maintained for $1 \mathrm{~h}$ at $0{ }^{\circ} \mathrm{C}$. The reaction was quenched by addition of $\mathrm{MeOH}$, filtered through a pad of celite and concentrated. The crude product was purified by flash chromatography (9:1 Tol/EtOAc) to give 9b as an off-white solid. $\mathrm{R}_{\mathrm{f}} 0.16$ (9:1 Tol/EtOAc). Yield: $33.1 \mathrm{~g}(91 \%)$. IR (neat, $\mathrm{cm}^{-1}$ ): 3481.62, 3090.41, 2951.69, 2870.27, 1756.92, 1479.38, 1406.63, 1365.59, 1313.58, 1288.85, 1166.86, 1044.29, 1025.82. ${ }^{1} \mathbf{H}$ NMR (400 MHz, $\left.\mathrm{CDCl}_{3}\right) \delta 7.65-7.57$ (m, 2H, Ar-H), $7.34-7.26$ (m, 8H, Ar-H), 5.40 (s, $\left.1 \mathrm{H},-\mathrm{CH}^{\text {benzylidene }}\right), 4.90$ (dd, $J_{2,3}$ = 9.6, $\left.J_{3,4}=3.4 \mathrm{~Hz}, 1 \mathrm{H}, \mathrm{H}-3\right), 4.51\left(\mathrm{~d}, J_{1,2}=9.6 \mathrm{~Hz}, 1 \mathrm{H}, \mathrm{H}-1\right), 4.35$ (dd, $\left.J_{3,4}=3.4, J_{4,5}=1.1 \mathrm{~Hz}, 1 \mathrm{H}, \mathrm{H}-4\right), 4.32\left(\mathrm{dd}, J_{6 a, 6 b}=12.5, J_{5,6 a}=\right.$ $1.6 \mathrm{~Hz}, 1 \mathrm{H}, \mathrm{H}-6 \mathrm{a}), 4.05$ (d, $\left.J_{\mathrm{CH} 2}=15.3 \mathrm{~Hz}, 1 \mathrm{H}, 0.5 \mathrm{xCH}_{2}{ }^{\mathrm{AcCl}}\right), 4.00$ (d, $J=15.3 \mathrm{~Hz}, 1 \mathrm{H}, 0.5 \mathrm{xCH}_{2}{ }^{\mathrm{AcCl}}$ ), 3.96 (dd, $J_{6 a, 6 b}=12.5, J_{5,6 b}=1.7$ $\mathrm{Hz}, 1 \mathrm{H}, \mathrm{H}-6 \mathrm{~b}$ ), 3.90 (td, $J_{1,2}=J_{2,3}=9.6, J_{2, \mathrm{OH}}=2.5 \mathrm{~Hz}, 1 \mathrm{H}, \mathrm{H}-2$ ), $3.55(\mathrm{~m}, 1 \mathrm{H}, \mathrm{H}-5), 2.34$ (d, $\left.J_{2, \mathrm{OH}}=2.5 \mathrm{~Hz}, 1 \mathrm{H}, \mathrm{OH}\right) .{ }^{13} \mathbf{C} \mathbf{N M R}$ (101 MHz, $\left.\mathrm{CDCl}_{3}\right) \delta 167.2,137.5,133.7$ (2C), 130.1, 129.2, 129.1 (2C), 128.4, 128.2 (2C), 126.4 (2C), 101.0, 87.5, 76.5, 73.3, 69.8, 69.1, 65.5, 40.9. HRMS (ESI-TOF) m/z: $[\mathrm{M}+\mathrm{Na}]^{+}$Calcd for $\mathrm{C}_{21} \mathrm{H}_{21} \mathrm{ClNaO}_{6} \mathrm{~S}$ 459.0645; Found 459.0639.

Phenyl 4,6-O-benzylidene-3-O-chloroacetyl-2-O-pivaloyl-1thio- $\beta$-D-galactopyranoside (10b). Compound 9b (25 g; 57.2 mmol) was dissolved in $\mathrm{CH}_{2} \mathrm{Cl}_{2}(400 \mathrm{~mL})$. Et $3 \mathrm{~N}(16.0 \mathrm{~mL} ; 114.4$ mmol), DMAP (3.5 g; $28.6 \mathrm{mmol}$ ) and pivaloyl chloride $(7.3 \mathrm{~mL}$; $85.8 \mathrm{mmol}$ ) was added to the solution and the reaction mixture was heated to $45^{\circ} \mathrm{C}$ for $4 \mathrm{~h}$. The reaction mixture was cooled to $0{ }^{\circ} \mathrm{C}$, quenched with $\mathrm{MeOH}(10 \mathrm{~mL})$, washed with water $(2 \times 400 \mathrm{~mL})$, dried over $\mathrm{MgSO}_{4}$ and concentrated. The product was purified by flash chromatography (15:1 Tol/EtOAc) to afford $\mathbf{1 0 b}$ as a white powder. $\mathrm{R}_{\mathrm{f}} 0.44$ (9:1 Tol/EtOAc). Yield $26.2 \mathrm{~g}$ (88\%). IR (neat, $\left.\mathrm{cm}^{-1}\right)$ : 3061.25, 3036.54, 2974.08, 2935.25, 2905.99, 2871.97, 1764.64, 1733.56, 1479.53, 1457.77, 1402.52, 1366.86, 1312.92, 1281.38, 1249.15, 1147.33, 1092.80, 1048.41, 1025.97, 997.24. ${ }^{1} \mathbf{H}$ NMR (400 MHz, $\left.\mathrm{CDCl}_{3}\right) \delta 7.56-7.47$ (m, 2H, Ar-H), 7.35 - 7.25 $(\mathrm{m}, 8 \mathrm{H}, \mathrm{Ar}-\mathrm{H}), 5.40\left(\mathrm{~s}, 1 \mathrm{H}, \mathrm{CH}^{\text {benzylidene }}\right), 5.28\left(\mathrm{t}, J_{1,2}=J_{2,3}=9.9 \mathrm{~Hz}\right.$, $1 \mathrm{H}, \mathrm{H}-2$ ), 5.07 (dd, $J_{2,3}=9.9, J_{3,4}=3.5 \mathrm{~Hz}, 1 \mathrm{H}, \mathrm{H}-3$ ), 4.67 (d, $J_{1,2}=$ $9.9 \mathrm{~Hz}, 1 \mathrm{H}, \mathrm{H}-1$ ), 4.31 (dd, $J_{6 a, 6 b}=11.5, J_{5,6 a}=0.9 \mathrm{~Hz}, 1 \mathrm{H}, \mathrm{H}-6 \mathrm{a}$ ), 4.30 (d, $J=3.5 \mathrm{~Hz}, 1 \mathrm{H}, \mathrm{H}-4), 3.96$ (dd, $J_{6 a, 6 b}=11.5, J_{5,6 b}=1.8 \mathrm{~Hz}$, $1 \mathrm{H}, \mathrm{H}-6 \mathrm{~b}), 3.95$ (d, $J_{\mathrm{CH} 2}=15.2 \mathrm{~Hz}, 1 \mathrm{H}, \mathrm{CH}_{2}{ }^{\mathrm{AcCl}}$ ), 3.87 (d, $J_{C H 2}=$ $15.2 \mathrm{~Hz}, 1 \mathrm{H}, \mathrm{CH}_{2}{ }^{\mathrm{AcCl}}$ ), 3.53 (s, $\left.1 \mathrm{H}, \mathrm{H}-5\right), 1.14$ (s, 9H, 3xCH${ }^{\mathrm{Piv}}$ ). ${ }^{13} \mathrm{C}$ NMR (101 MHz, $\left.\mathrm{CDCl}_{3}\right) \delta 176.5,167.1,137.4,133.8(2 \mathrm{C})$, 131.4, 129.4, 128.9 (2C), 128.4, 128.3 (2C), 126.6 (2C), 101.2, 85.4, 74.8, 73.5, 69.7, 69.2, 66.2, 40.7, 38.9, 27.2 (3C). HRMS (ESI-TOF) m/z: [M + NH4] ${ }^{+}$Calcd for $\mathrm{C}_{26} \mathrm{H}_{33} \mathrm{ClNO}_{7} \mathrm{~S} 538.1666$ Found 538.1671.

4,6-O-benzylidene-3-O-chloroacetyl-2-O-pivaloyl-D-galactopyranose (11b). Compound 10b (20.0 g; $38.4 \mathrm{mmol})$ was dissolved in acetone $(180 \mathrm{~mL})$ and water $(20 \mathrm{~mL})$. NBS (27.3 g; $153.5 \mathrm{mmol})$ and 2,4-lutidine (22.2 $\mathrm{mL}$; $107.2 \mathrm{mmol})$ were added and the reaction was stirred at $50{ }^{\circ} \mathrm{C}$ until TLC showed full conversion (3h). 
The solution was diluted with $\mathrm{CH}_{2} \mathrm{Cl}_{2}(500 \mathrm{~mL})$ and washed with sat. aq. $\mathrm{NaS}_{2} \mathrm{O}_{3}(200 \mathrm{~mL})$ and sat. aq. $\mathrm{NaHCO}_{3}(200 \mathrm{~mL})$. The organic phase was dried over MgSO4, filtered and concentrated. The product was purified by flash chromatography (6:1 toluene/EtOAc) to afford $11 b$ as an $\alpha / \beta$ mixture. Rf 0.13 (9:1 toluene/EtOAc). Yield: 12.5 g (76\%). IR (neat, $\left.\mathrm{cm}^{-1}\right): 3063.99,3030.86,2972.24,2931.14$, 2870.06, 1738.21, 1479.47, 1454.40, 1365.98, 1277.87, 1150.65, 1134.03, 1101.35, 1064.79. ${ }^{1} \mathbf{H}$ NMR (400 MHz, $\left.\mathrm{CDCl}_{3}\right) \delta 7.49-$ 7.03 (m, 10H, Ar-H), 5.51 (d, $J_{1,2}=3.5 \mathrm{~Hz}, 1 \mathrm{H}, \mathrm{H}-1 \alpha$ ), 5.45 (dd, $\left.J_{2,3}=10.1, J_{3,4}=2.9 \mathrm{~Hz}, 1 \mathrm{H}, \mathrm{H}-3 \alpha\right), 5.43\left(\mathrm{~s}, 1 \mathrm{H}, \mathrm{H}^{\text {benzylidene }} \beta\right), 5.41$ (s, $1 \mathrm{H}, \mathrm{H}^{\text {benzylidene }} \alpha$ ), 5.18 (dd, $J_{2,3}=10.1, J_{1,2}=3.5 \mathrm{~Hz}, 1 \mathrm{H}, \mathrm{H}-2 \alpha$ ), 5.13 (dd, $J_{2,3}=10.4, J_{1,2}=7.9 \mathrm{~Hz}, 1 \mathrm{H}, \mathrm{H}-2 \beta$ ), 5.02 (dd, $J_{2,3}=10.4$, $J_{3,4}=3.6 \mathrm{~Hz}, 1 \mathrm{H}, \mathrm{H}-3 \beta$ ), 4.60 (d, $J_{1,2}=7.9 \mathrm{~Hz}, 1 \mathrm{H}, \mathrm{H}-1 \beta$ ), 4.32 (dd, $\left.J_{3,4}=2.9, J_{4,5}=1.1 \mathrm{~Hz}, 1 \mathrm{H}, \mathrm{H}-4 \alpha\right), 4.29-4.22(\mathrm{~m}, 1 \mathrm{H}, \mathrm{H}-4 \mathrm{~b}, \mathrm{H}-$ $6 \mathrm{a}, \beta) 4.16$ (dd, $\left.J_{6 a, 6 b}=12.6, J_{5,6 a}=1.5 \mathrm{~Hz}, 1 \mathrm{H}, \mathrm{H}-6 \mathrm{a}, \alpha\right), 4.01$ (d, $\left.J_{C H 2}=15.0 \mathrm{~Hz}, 1 \mathrm{H}, 0.5 \mathrm{xCH}_{2}{ }^{\mathrm{AcCl}}\right), 3.94(\mathrm{~m}, 3 \mathrm{H}, \mathrm{H}-6 \mathrm{~b}, \alpha ; \mathrm{H}-6 \mathrm{~b}, \beta$; $0.5 \mathrm{xCH}_{2}{ }^{\mathrm{AcCl}}$ ), 3.91 (m, $\left.2 \mathrm{H}, \mathrm{H}-5 \alpha, \mathrm{H}-5 \beta\right), 1.11$ (m, $18 \mathrm{H}, 6 \mathrm{xCH}_{3}{ }^{\mathrm{Piv}}$ ). ${ }^{13} \mathrm{C}$ NMR (101 MHz, $\left.\mathrm{CDCl}_{3}\right) \delta 177.7,176.9,165.9,136.8,136.4$, 136.2, 128.2, 128.1, 128.0, 127.3, 127.22, 127.19, 125.2, 125.1, 124.3, 99.7, 99.6, 94.8, 89.8, 72.9, 72.2, 71.9, 69.5, 69.0, 68.1, 67.9, 67.2, 65.4, 60.9, 39.7, 39.5, 37.9, 37.8, 25.9. HRMS (ESI-TOF) $\mathrm{m} / \mathrm{z}:\left[\mathrm{M}+\mathrm{NH}_{4}\right]^{+}$Calcd for $\mathrm{C}_{20} \mathrm{H}_{30} \mathrm{ClNO}_{8} 447.1660$ Found 447.1671.

4,6-O-Benzylidene-3-O-chloroacetyl-2- $O$-pivaloyl-1-thio- $\beta$-Dgalactopyranose $N$-phenyl trifluoroacetimidate (12b). Compound 11b (10 g; $23.3 \mathrm{mmol})$ was dissolved in $\mathrm{CH}_{2} \mathrm{Cl}_{2}(230 \mathrm{~mL})$ and cooled to $0{ }^{\circ} \mathrm{C}$. $\mathrm{Cs}_{2} \mathrm{CO}_{3}(15.2 \mathrm{~g} ; 46.6 \mathrm{mmol}$ ) was added followed by $N$-phenyl trifluoroacetimidoyl chloride (9.7 g; 46.6 mmol). The ice bath was removed and the reaction mixture was stirred until TLC showed full conversion (5 h). It was then filtered, concentrated and purified by flash chromatography to give an offwhite foam. Yield: $11.2 \mathrm{~g}(80 \%)$.

Phenyl 4,6- $O$-benzylidene-2- $O$-pivaloyl-1-thio- $\beta$-D-galactopyranoside (14). Compound 10b (10 g; $19.2 \mathrm{mmol})$ was dissolved in dry $\mathrm{CH}_{2} \mathrm{Cl}_{2}(190 \mathrm{~mL})$ and cooled to $0{ }^{\circ} \mathrm{C}$. A $1 \mathrm{M} \mathrm{L}$-selectride solution in THF $(57.6 \mathrm{~mL})$ was added and the reaction was stirred at 0 ${ }^{\circ} \mathrm{C}$ until complete consumption of the starting material $(1 \mathrm{~h})$. The reaction mixture was poured into sat. aq. $\mathrm{NH}_{4} \mathrm{Cl}(400 \mathrm{~mL})$. The organic phase was dried over $\mathrm{MgSO}_{4}$, filtered and concentrated (avoid concentrating to dryness since the borane salts can be explosive). The crude product was purified by flash chromatography to give 14 as a white solid. Yield: $8.2 \mathrm{~g}(96 \%) .{ }^{1} \mathbf{H}$ NMR (400 MHz, $\left.\mathrm{CDCl}_{3}\right) \delta 7.51(\mathrm{~m}, 2 \mathrm{H}, \mathrm{Ar}-\mathrm{H}), 7.38-7.10$ (m, 10H, Ar-H), 5.45 (s, $\left.1 \mathrm{H}, \mathrm{CH}^{\text {benzylidene }}\right), 4.98\left(\mathrm{t}, J_{1,2}=J_{2,3}=9.7 \mathrm{~Hz}, 1 \mathrm{H}, \mathrm{H}-2\right), 4.60$ (d, $J_{1,2}$ $=9.7 \mathrm{~Hz}, 1 \mathrm{H}, \mathrm{H}-1$ ), 4.31 (dd, $J_{6 a, 6 b}=12.5, J_{5,6 a}=1.5 \mathrm{~Hz}, 1 \mathrm{H}, \mathrm{H}-6 \mathrm{a}$ ), 4.14 (dd, $\left.J_{3,4}=3.6, J_{4,5}=1.1 \mathrm{~Hz}, 1 \mathrm{H}, \mathrm{H}-4\right), 3.96$ (dd, $J_{6 a, 6 b}=12.5$, $J_{5,6 b}=1.7 \mathrm{~Hz}, 1 \mathrm{H}, \mathrm{H}-6 \mathrm{~b}$ ), 3.67 (dd, $J_{2,3}=9.7, J_{3,4}=3.6 \mathrm{~Hz}, 1 \mathrm{H}, \mathrm{H}-$ 3), 3.51 - 3.43 (m, 1H, H-5), 1.19 (s, 9H, $3 \mathrm{xCH}_{3}{ }^{\text {Piv }}$ ). ${ }^{13} \mathrm{C}$ NMR (101 $\left.\mathrm{MHz}, \mathrm{CDCl}_{3}\right) \delta 177.6,137.4,133.6$ (2C), 131.6, 129.4, 128.8 (2C), 128.2 (2C), 128.1, 126.5 (2C), 101.4, 85.1, 75.7, 72.9, 69.9, 69.5, 69.2, 38.8, 27.2 (3C). HRMS (ESI-TOF) m/z: [M + Na] ${ }^{+}$Calcd for $\mathrm{C}_{24} \mathrm{H}_{28} \mathrm{NaO}_{6} \mathrm{~S}$ : 467.1504; Found 467.1511.

\footnotetext{
Phenyl 4,6-O-benzylidene-3- $O$-chloroacetyl-2- $O$-pivaloyl- $\beta$-Dgalactopyranosyl-( $\rightarrow 3)-4,6-O$-benzylidene-2- $O$-pivaloyl-1-

thio- $\boldsymbol{\beta}$-D-galactopyranoside (15b). To a $250 \mathrm{~mL}$ flame-dried flask was added 14 (6.5 g, $14.6 \mathrm{mmol})$ and the $\mathbf{1 2 b}$ (11.4 g, $19.0 \mathrm{mmol})$. The mixture was co-evaporated with toluene $(2 \times 150 \mathrm{~mL})$ and subjected to vacuum overnight. The mixture dissolved in $\mathrm{CH}_{2} \mathrm{Cl}_{2}$ $(150 \mathrm{~mL})$ and cooled to $-40^{\circ} \mathrm{C}$. TMSOTf $(0.22 \mathrm{~mL} ; 1.5 \mathrm{mmol})$ was added and the reaction mixture was stirred at $-40{ }^{\circ} \mathrm{C}$ for $2 \mathrm{~h}$. Et $3 \mathrm{~N}$ ( $1 \mathrm{~mL}$ ) was added and the reaction mixture was concentrated. The crude compound was purified by flash chromatography (9:1 Tol/EtOAc) affording 15b. Rf 0.26 (9:1 Tol/EtOAc). Yield: $10.5 \mathrm{~g}$
}

(84\%). ${ }^{1} \mathbf{H}$ NMR (400 MHz, $\left.\mathrm{CDCl}_{3}\right) \delta 7.51-7.36$ (m, 5H), $7.35-$ $7.22(\mathrm{~m}, 6 \mathrm{H}), 7.17-7.04(\mathrm{~m}, 3 \mathrm{H}), 5.49\left(\mathrm{~s}, 1 \mathrm{H},-\mathrm{CH}^{\text {benzylidene }}\right), 5.43$ (s, $1 \mathrm{H},-\mathrm{CH}^{\text {benzylidene }}$ ), 5.33 (dd, $J_{2,3}=9.7 J_{1,2}=8.1 \mathrm{~Hz}, 1 \mathrm{H}, \mathrm{H}-2^{\prime}$ ), 5.30 (t, $\left.J_{1,2}=J_{2,3}=9.7 \mathrm{~Hz}, 1 \mathrm{H}, \mathrm{H}-2\right), 4.87$ (d, $J=8.1 \mathrm{~Hz}, 1 \mathrm{H}, \mathrm{H}-1^{\prime}$ ), 4.86 (d, $J=3.8 \mathrm{~Hz}, 1 \mathrm{H}), 4.60$ (d, $\left.J_{1,2}=9.7 \mathrm{~Hz}, 1 \mathrm{H}, \mathrm{H}-1\right), 4.34-$ 4.17 (m, 4H, H-3, H-4, H-4', H-6a'), 4.00 (dd, $J=12.5,1.5$ Hz, $1 \mathrm{H}$, H-6a), 3.99 (d, $J_{C H 2}=15.3 \mathrm{~Hz}, 1 \mathrm{H}, \mathrm{CH}_{2}{ }^{\mathrm{AcCl}}$ ), $3.98-3.89$ (dd, $J_{6 a, 6 b}$ $\left.=12.5 \mathrm{~Hz}, J_{5,6 b}=1.5,1 \mathrm{H}, \mathrm{H}-6 \mathrm{~b}\right), 3.93\left(\mathrm{dd}, J_{6 a, 6 b}=12.3 \mathrm{~Hz}, J_{5,6 b}\right.$ $=1.1,1 \mathrm{H}, \mathrm{H}-6 \mathrm{~b}$ '), 3.91 (d, $\left.J_{\mathrm{CH} 2}=15.2 \mathrm{~Hz}, 1 \mathrm{H}, \mathrm{CH}_{2}{ }^{\mathrm{AcCl}}\right), 3.38(\mathrm{~m}$, 2H, H-5, H-5'), 1.22 (s, 9H, - $\mathrm{CH}_{3}{ }^{\mathrm{Piv}}$ ), 1.00 (s, 9H, $\left.-\mathrm{CH}_{3}{ }^{\mathrm{Piv}}\right) .{ }^{13} \mathrm{C}$ NMR (101 MHz, $\left.\mathrm{CDCl}_{3}\right) \delta 176.9,176.1,167.1,137.8,137.3$, 133.4, 132.3 (2C), 129.2, 128.7 (2C), 128.6, 128.3 (2C), 127.9 (2C), 127.6, 126.22 (2C), 126.19 (2C), 100.9, 100.2, 99.3, 86.8, 75.9, 73.6, 73.5, 73.3, 70.2, 69.5, 68.9, 68.7, 68.2, 66.6, 40.6, 38.79, 38.76, 27.4 (3C), 27.0 (3C). HRMS (ESI-TOF) m/z: [M + $\left.\mathrm{NH}_{4}\right]^{+}$ Calcd for $\mathrm{C}_{44} \mathrm{H}_{55} \mathrm{ClNO}_{13} \mathrm{~S}$ 872.3083 Found 872.3092.

Phenyl 4,6-O-benzylidene-2,3-di- $O$-pivaloyl- $\beta$-D-galactopyranosyl-( $\rightarrow 3$ )-4,6- $O$-benzylidene-2- $O$-pivaloyl-1-thio- $\beta$-D-galactopyranoside (20). Compound $15 \mathrm{~b}(1.8 \mathrm{~g} ; 2.1 \mathrm{mmol})$ was dissolved in dry $\mathrm{CH}_{2} \mathrm{Cl}_{2}(40 \mathrm{~mL})$ and cooled to $0{ }^{\circ} \mathrm{C}$. A $1 \mathrm{M} \mathrm{L}$-selectride solution in THF $(5.3 \mathrm{~mL})$ was added and the reaction was stirred at $0{ }^{\circ} \mathrm{C}$ until complete consumption of the starting material was observed (30 $\mathrm{min})$. The reaction mixture was quenched with sat. aq. $\mathrm{NH}_{4} \mathrm{Cl}(50 \mathrm{~mL})$. The organic phase was dried over $\mathrm{MgSO}_{4}$, filtered and concentrated (avoid concentrating to dryness since the borane salts can be explosive). The residue was filtered through a plug of silica (9:1 Tol/EtOAc). The semi-crude alcohol (1.4 g; 1.8 mmol) was dissolved in $\mathrm{CH}_{2} \mathrm{Cl}_{2}(20 \mathrm{~mL})$. $\mathrm{Et}_{3} \mathrm{~N}(0.5 \mathrm{~mL}$; $3.6 \mathrm{mmol})$, DMAP (0.1 g; $0.9 \mathrm{mmol})$ and pivaloyl chloride (0.4 mL; $3.6 \mathrm{mmol})$ was added to the solution and the reaction mixture was heated to $45^{\circ} \mathrm{C}$ for $4 \mathrm{~h}$. The reaction mixture was cooled to $0{ }^{\circ} \mathrm{C}$, quenched with $\mathrm{MeOH}(0.5 \mathrm{~mL})$, washed with water $(2 \times 50 \mathrm{~mL})$, dried over $\mathrm{MgSO}_{4}$ and concentrated. The product was purified by flash chromatography (Tol/EtOAc 9:1) to afford $\mathbf{2 0}$ as an off-white solid. $\mathrm{R}_{\mathrm{f}}$ 0.26 (9:1 Tol/EtOAc). Yield $1.3 \mathrm{~g}$ (80\% over two steps). IR (neat, $\left.\mathrm{cm}^{-1}\right)$ : 3065.46, 2933.04, 1734.90, 1479.30, 1455.98, 1397.64, 1366.82, 1279.26, 1173.33, 1140.59, 1091.22, 1048.23, 1026.27. ${ }^{1} \mathbf{H}$ NMR (400 MHz, $\left.\mathrm{CDCl}_{3}\right) \delta 7.49-7.34(\mathrm{~m}, 6 \mathrm{H}, \mathrm{Ar}-\mathrm{H}), 7.26(\mathrm{~m}$, 9H, Ar-H), 5.47 (s, 1H, -CH $\left.{ }^{\text {benzylidene }}\right), 5.45$ (s, $\left.1 \mathrm{H},-\mathrm{CH}^{\text {benzylidene }}\right)$, 5.37 (dd, $J_{2,3}=10.5, J_{1,2}=8.0 \mathrm{~Hz}, 1 \mathrm{H}, \mathrm{H}-2$ '), 5.29 (t, $J_{1,2}=J_{2,3}=9.8$ $\mathrm{Hz}, 1 \mathrm{H}, \mathrm{H}-2$ ), 4.81 (d, $J_{1,2}=8.0 \mathrm{~Hz}, 1 \mathrm{H}, \mathrm{H}-1^{\prime}$ ), 4.69 (dd, $J_{2,3}=10.5$, $J_{3,4}=3.7 \mathrm{~Hz}, 1 \mathrm{H}, \mathrm{H}-3$ '), 4.60 (d, $J_{1,2}=9.8 \mathrm{~Hz}, 1 \mathrm{H}, \mathrm{H}-1$ ), $4.38-4.17$ (m, 5H, H-3, H-4, H-4', H-6a, H-6a'), 4.01 (dd, $J_{6 a, 6 b}=12.5, J_{5,6 b}$ $=1.8 \mathrm{~Hz}, 1 \mathrm{H}, \mathrm{H}-6 \mathrm{~b}$ '), 3.93 (dd, $J_{6 a, 6 b}=12.4, J_{5,6 b}=1.6 \mathrm{~Hz}, 1 \mathrm{H}, \mathrm{H}-$ 6b), $3.42-3.34$ (m, 2H, H-5, H-5'), 1.22 (s, 9H, 3xCH ${ }_{3}^{\mathrm{Piv}}$ ), 1.07 (s, $\left.9 \mathrm{H}, 3 \mathrm{xCH}_{3}{ }^{\mathrm{Piv}}\right), 0.98$ (s, 9H, $\left.3 \mathrm{xCH}_{3}{ }^{\mathrm{Piv}}\right) .{ }^{13} \mathrm{C}$ NMR $(101 \mathrm{MHz}$, $\left.\mathrm{CDCl}_{3}\right) \delta 178.2,176.8,176.1,137.9,137.5,133.4,132.3(2 \mathrm{C})$, 128.9, 128.7 (2C), 128.5, 128.2 (2C), 127.9 (2C), 127.5, 126.2 (2C), 125.9 (2C), 100.4, 100.1, 99.3, 86.7, 75.8, 73.1 (2C), 71.9, 70.2, 69.7, 68.9, 68.8, 68.2, 66.8, 38.9, 38.8, 38.7, 27.4, 27.00, 26.98. HRMS (ESI-TOF) m/z: [M+Na] ${ }^{+}$Calcd for $\mathrm{C}_{47} \mathrm{H}_{58} \mathrm{NaO}_{13} \mathrm{~S}$ 885.3496; Found 885.3499.

Phenyl 6-O-acetyl-4-O-benzyl-3-O-chloroacetyl-2-O-pivaloyl1-thio- $\beta$-D-galactopyranoside (21). A solution of $1 \mathrm{M} \mathrm{BH}_{3}$ in THF $(76.7 \mathrm{~mL})$ was added to a $250 \mathrm{ml}$ dry flask containing $10 \mathrm{~b}(4.0 \mathrm{~g}$; $7.7 \mathrm{mmol})$ at $0{ }^{\circ} \mathrm{C}$ and the solution was stirred for 5 minutes. A solution of $1 \mathrm{M} \mathrm{Cu}(\mathrm{OTf})_{2}$ in $\mathrm{CH}_{2} \mathrm{Cl}_{2}(8.1 \mathrm{~mL})$ was then added to the clear solution slowly. After 1.5 hours at $0{ }^{\circ} \mathrm{C}$, TLC showed full consumption of starting material. Triethylamine $(2 \mathrm{~mL})$ was added followed by careful addition of methanol until the evolution of $\mathrm{H}_{2}$ had ceased. The reaction mixture was co-distilled with methanol three times and the crude was used in the next step. The crude material was dissolved in $\mathrm{CH}_{2} \mathrm{Cl}_{2}(50 \mathrm{~mL})$. Et $3 \mathrm{~N}$ (1.1 mL; $\left.7.7 \mathrm{mmol}\right)$, DMAP (0.01 g; $0.1 \mathrm{mmol})$ and acetic anhydride $(0.61 \mathrm{~mL}$; $6.5 \mathrm{mmol}$ ) was added to the solution and the reaction mixture was 
stirred until TLC revealed full conversion $(1 \mathrm{~h})$. The reaction was quenched with $\mathrm{MeOH}(1 \mathrm{~mL})$, diluted with $\mathrm{CH}_{2} \mathrm{Cl}_{2}(50 \mathrm{~mL})$, washed with water $(2 \times 100 \mathrm{~mL})$, dried over $\mathrm{MgSO}_{4}$ and concentrated. The product was purified by flash chromatography (Tol/EtOAc 15:1) to afford 21 as an off-white solid. $\mathrm{R}_{\mathrm{f}} 0.42$ (9:1 Tol/EtOAc). Yield: $2.8 \mathrm{~g}$ (68\%) over two steps. IR (neat, $\mathrm{cm}^{-1}$ ): 3061.37, 3031.47, 2972.29, 2906.59, 2872.68, 1739.50, 1584.17, 1496.32, 1479.63, 1455.92, 1440.05, 1398.67, 1369.01, 1277.91, 1232.53, 1172.17, 1139.11, 1088.80, 1043.02. ${ }^{\mathbf{1}} \mathbf{H} \quad \mathbf{N M R}$ (400 MHz, $\left.\mathrm{CDCl}_{3}\right) \delta 7.47$ - 7.37 (m, 2H), $7.33-7.15$ (m, 8H), 5.34 (t, $\left.J_{1,2}=J_{2,3}=9.9 \mathrm{~Hz}, 1 \mathrm{H}, \mathrm{H}-2\right), 5.06$ (dd, $J_{2,3}=9.9, J_{3,4}=3.0 \mathrm{~Hz}$, $1 \mathrm{H}, \mathrm{H}-3), 4.62$ (d, $\left.J_{1,2}=9.9 \mathrm{~Hz}, 1 \mathrm{H}, \mathrm{H}-1\right), 4.63\left(\mathrm{~d}, J_{\mathrm{CH} 2}=11.9 \mathrm{~Hz}\right.$, $\left.1 \mathrm{H}, \mathrm{CH}_{2}{ }^{\mathrm{Bn}}\right), 4.53\left(\mathrm{~d}, J_{\mathrm{CH} 2}=11.9 \mathrm{~Hz}, 1 \mathrm{H}, \mathrm{CH}_{2}{ }^{\mathrm{Bn}}\right), 4.26\left(\mathrm{dd}, J_{6 a, 6 b}=\right.$ 11.2, $\left.J_{5,6 a}=6.7 \mathrm{~Hz}, 1 \mathrm{H}, \mathrm{H}-6 \mathrm{a}\right), 4.04$ (dd, $J_{6 a, 6 b}=11.2, J_{5,6 b}=6.2 \mathrm{~Hz}$, $1 \mathrm{H}, \mathrm{H}-6 \mathrm{~b}$ ), 3.88 (dd, $J_{3,4}=3.0, J_{4,5}=1.0 \mathrm{~Hz}, 1 \mathrm{H}, \mathrm{H}-4$ ), 3.80 (d, $J=$ $\left.14.9 \mathrm{~Hz}, 1 \mathrm{H}, 0.5 \mathrm{xCH}_{2}{ }^{\mathrm{AcCl}}\right), 3.74\left(\mathrm{~d}, J=15.0 \mathrm{~Hz}, 1 \mathrm{H}, 0.5 \mathrm{xCH}_{2}{ }^{\mathrm{AcCl}}\right)$, $3.72-3.67(\mathrm{~m}, 1 \mathrm{H}, \mathrm{H}-5), 1.95\left(\mathrm{~s}, 3 \mathrm{H},-\mathrm{CH}_{3}{ }^{\mathrm{Ac}}\right), 1.13(\mathrm{~s}, 9 \mathrm{H}$, $\left.3 \mathrm{xCH}_{3}{ }^{\mathrm{Piv}}\right) .{ }^{13} \mathrm{C}$ NMR $\left(101 \mathrm{MHz}, \mathrm{CDCl}_{3}\right) \delta 176.8,170.5,166.8$, 137.4, 133.0, 132.6 (2C), 129.0 (2C), 128.6 (2C), 128.4 (2C), 128.2, 128.1, 86.9, 76.5, 76.0, 75.1, 73.9, 67.4, 62.5, 40.4, 38.9, 27.2 (3C), 20.9. HRMS (ESI-TOF) $\mathrm{m} / \mathrm{z}:[\mathrm{M}+\mathrm{Na}]^{+}$Calcd for $\mathrm{C}_{28} \mathrm{H}_{33} \mathrm{ClNaO}{ }_{8} \mathrm{~S}$ 587.1482; Found 587.1481.

Benzyl 6-O-acetyl-4-O-benzyl-3-O-chloroacetyl-2- $O$-pivaloyl$\beta$-D-galactopyranosyl-(1 $\rightarrow 3)$-4,6-O-benzylidene-2-O-pivaloyl$\beta$-D-galactopyranosyl-( $\rightarrow 3$ )-4,6-O-benzylidene-2-O-pivaloyl$\boldsymbol{\beta}$-D-galactopyranoside (22). To a $25 \mathrm{~mL}$ flame-dried flask was added 16 (3.0 g, $3.9 \mathrm{mmol}$ ) and the 21 (1.5 g, $1.8 \mathrm{mmol}$ ). The mixture was dried azeotropically with toluene $(2 \times 10 \mathrm{~mL})$ and subjected to vacuum overnight. It was then dissolved in dry $\mathrm{CH}_{2} \mathrm{Cl}_{2}(5 \mathrm{~mL})$ and dry MeCN (5 mL), cooled to $-30{ }^{\circ} \mathrm{C}$, followed by addition of NIS (420 mg; $1.9 \mathrm{mmol}$ ) and TESOTf (56 mg; $0.2 \mathrm{mmol}$ ). The reaction mixture was stirred at $-30{ }^{\circ} \mathrm{C}$ until TLC revealed full conversion of the donor $(2 \mathrm{~h})$. The solution was diluted with $\mathrm{CH}_{2} \mathrm{Cl}_{2}$ $(100 \mathrm{~mL})$ and washed with sat. aq. $\mathrm{NaS}_{2} \mathrm{O}_{3}(100 \mathrm{~mL})$ and sat. aq. $\mathrm{NaHCO}_{3}(100 \mathrm{~mL})$. The organic phase was dried over $\mathrm{MgSO}_{4}$, filtered and concentrated. The product was purified by flash chromatography (6:1 Tol/EtOAc) to afford an off-white solid. $\mathrm{R}_{\mathrm{f}} 0.60$ (2:1 Tol/EtOAc). Yield: 3.6 g (75\%). ${ }^{1} \mathbf{H}$ NMR (400 MHz, $\left.\mathrm{CDCl}_{3}\right) \delta$

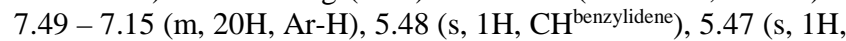
$\mathrm{CH}^{\text {benzylidene }}$ ), 5.37 (dd, $J_{2,3}=10.2, J_{1,2}=7.9 \mathrm{~Hz}, 1 \mathrm{H}, \mathrm{H}-2^{1}$ ), 5.31 (dd, $J_{2,3}=10.3, J_{1,2}=8.0 \mathrm{~Hz}, 1 \mathrm{H}, \mathrm{H}-2^{3}$ ), $5.27\left(\mathrm{dd}, J_{2,3}=10.2, J_{1,2}=7.7\right.$ $\mathrm{Hz}, 1 \mathrm{H}, \mathrm{H}-2^{2}$ ), 4.88 (dd, $J_{2,3}=10.3, J_{3,4}=3.0 \mathrm{~Hz}, 1 \mathrm{H}, \mathrm{H}-3^{3}$ ), 4.82 (d, $J_{\mathrm{CH} 2}=11.9 \mathrm{~Hz}, 1 \mathrm{H}, 0.5 \mathrm{xCH}_{2}{ }^{\mathrm{Bn}}$ ), $4.73\left(\mathrm{~d}, J_{1,2}=7.7 \mathrm{~Hz}, 1 \mathrm{H}, \mathrm{H}-\right.$ $\left.1^{2}\right), 4.70\left(\mathrm{~d}, J_{1,2}=8.0 \mathrm{~Hz}, 1 \mathrm{H}, \mathrm{H}-1^{3}\right), 4.63\left(\mathrm{~d}, J_{\mathrm{CH} 2}=11.7 \mathrm{~Hz}, 1 \mathrm{H}\right.$, $\left.0.5 \mathrm{xCH}_{2}{ }^{\mathrm{Bn}}\right), 4.51\left(\mathrm{~d}, J_{\mathrm{CH} 2}=11.7 \mathrm{~Hz}, 1 \mathrm{H}, 0.5 \mathrm{XCH}_{2}{ }^{\mathrm{Bn}}\right), 4.45\left(\mathrm{~d}, J_{\mathrm{CH} 2}\right.$ $\left.=11.9 \mathrm{~Hz}, 1 \mathrm{H}, 0.5 \mathrm{xCH}_{2}{ }^{\mathrm{Bn}}\right), 4.36\left(\mathrm{~d}, J_{1,2}=7.9 \mathrm{~Hz}, 1 \mathrm{H}, \mathrm{H}-1^{1}\right), 4.32$ - 3.94 (m, 12H, H-3 $\left.{ }^{1}, \mathrm{H}-3^{2}, \mathrm{H}-4^{1}, \mathrm{H}-4^{2}, 3 \times \mathrm{H}-6\right), 3.86$ (d, $J=3.0$ $\mathrm{Hz}, 1 \mathrm{H}, \mathrm{H}-4^{3}$ ), 3.79 (d, $\left.J_{C H 2}=15.0 \mathrm{~Hz}, 1 \mathrm{H}, \mathrm{CH}_{2}{ }^{\mathrm{AcCl}}\right), 3.72\left(\mathrm{~d}, J_{C H}\right.$ $\left.=15.0 \mathrm{~Hz}, 1 \mathrm{H}, \mathrm{CH}_{2}{ }^{\mathrm{AcCl}}\right), 3.60\left(\mathrm{t}, J 5,6 a=J_{5,6 b}=6.6 \mathrm{~Hz}, 1 \mathrm{H}, \mathrm{H}-5^{3}\right)$, 3.31 (s, $\left.1 \mathrm{H}, \mathrm{H}-5^{1 / 2}\right), 3.29$ (s, $\left.1 \mathrm{H}, \mathrm{H}-5^{1 / 2}\right), 1.95$ (s, $\left.3 \mathrm{H}, \mathrm{CH}_{3}{ }^{\mathrm{Ac}}\right), 1.09$ (s, 9H, $\left.\mathrm{CH}_{3}{ }^{\mathrm{Piv}}\right), 1.05$ (s, 9H, $\mathrm{CH}_{3}{ }^{\mathrm{Piv}}$ ), 1.00 (s, 9H, $\left.\mathrm{CH}_{3}{ }^{\mathrm{Piv}}\right) \cdot{ }^{13} \mathbf{C}$ NMR $\left(101 \mathrm{MHz}, \mathrm{CDCl}_{3}\right) \delta 177.2,176.11,176.10,170.3,166.84,138.0$, 137.8, 137.32, 137.30, 128.8-126.1 (20C), 100.4, 100.3 (2C), 99.9, 99.7, 75.83, 75.77, 75.2, 75.0, 73.6, 72.6 (2C), 71.9, 71.8, 71.1, 70.0, 69.0, 68.9, 68.8, 67.4, 67.0, 62.0, 40.4, 38.9, 38.8, 38.7, 27.31 (3C), 27.25 (3C), 27.1 (3C), 20.9. HRMS (ESI-TOF) m/z: $\left[\mathrm{M}+\mathrm{NH}_{4}\right]^{+}$Calcd for $\mathrm{C}_{64} \mathrm{H}_{81} \mathrm{ClNO}_{20} 1250.4761$ Found 1250.4763 .

\footnotetext{
Benzyl 6-O-acetyl-4-O-benzyl-2-O-pivaloyl- $\beta$-D-galactopyranosyl-( $1 \rightarrow 3)-4,6$ - $O$-benzylidene-2- $O$-pivaloyl- $\beta$-D-galactopyranosyl- $(1 \rightarrow 3)-4,6$ - $O$-benzylidene-2- $O$-pivaloyl- $\beta$-D-galactopyranoside (23). Compound 22 (1.6 g; $1.3 \mathrm{mmol}$ ) was dissolved in 25 mL dry THF. Thiourea (382 mg; 5.0 mmol), BunNI (93 mg; 0.25 mmol) and $\mathrm{NaHCO}_{3}$ (464 mg; $5.5 \mathrm{mmol}$ ) was added and the reaction mixture was heated to $55^{\circ} \mathrm{C}$ for $12 \mathrm{~h}$. The mixture was filtered, concentrated and purified by flash chromatography (4:1
}

Tol/EtOAc) to give $\mathbf{2 3}$ as a yellowish amorphous material. $\mathrm{R}_{\mathrm{f}} 0.49$ (2:1 Tol/EtOAc). Yield: 1.2 g (82\%). IR (neat, $\left.\mathrm{cm}^{-1}\right): 3521.34$, 2973.04, 2932.85, 2907.14, 2872.47, 1737.38, 1479.63, 1454.83, 1397.45, 1366.55, 1277.19, 1231.25, 1133.51, 1078.77, 1061.55, 1044.64. ${ }^{1} \mathbf{H}$ NMR $\left(400 \mathrm{MHz}, \mathrm{CDCl}_{3}\right) \delta 7.50-7.14(\mathrm{~m}, 20 \mathrm{H}), 5.49$ (s, $1 \mathrm{H}, \mathrm{CH}^{\text {benzylidene }}$ ), 5.45 (s, $\left.1 \mathrm{H}, \mathrm{CH}^{\text {benzylidene }}\right), 5.39$ (dd, $J_{2,3}=10.2$, $\left.J_{1,2}=7.9 \mathrm{~Hz}, 1 \mathrm{H}, \mathrm{H}-2^{1}\right), 5.36\left(\mathrm{dd}, J_{2,3}=10.4, J_{1,2}=8.0 \mathrm{~Hz}, 1 \mathrm{H}, \mathrm{H}-\right.$ $2^{2}$ ), $4.85\left(\mathrm{dd}, J_{2,3}=10.0, J_{1,2}=7.7 \mathrm{~Hz}, 1 \mathrm{H}, \mathrm{H}-2^{3}\right), 4.83\left(\mathrm{~d}, J_{\mathrm{CH} 2}=\right.$ $\left.11.9 \mathrm{~Hz}, 1 \mathrm{H}, 0.5 \mathrm{xCH}_{2}{ }^{\mathrm{Bn}}\right), 4.76\left(\mathrm{~d}, J_{\mathrm{CH} 2}=11.5 \mathrm{~Hz}, 1 \mathrm{H}, 0.5 \mathrm{xCH}_{2}{ }^{\mathrm{Bn}}\right)$, $4.70\left(\mathrm{~d}, J_{1,2}=8.0 \mathrm{~Hz}, 1 \mathrm{H}, \mathrm{H}-1^{2}\right), 4.65\left(\mathrm{~d}, J_{1,2}=7.7 \mathrm{~Hz}, 1 \mathrm{H}, \mathrm{H}-1^{3}\right)$, $4.61\left(\mathrm{~d}, J_{\mathrm{CH} 2}=11.5 \mathrm{~Hz}, 1 \mathrm{H}, 0.5 \mathrm{xCH}_{2}{ }^{\mathrm{Bn}}\right), 4.45\left(\mathrm{~d}, J_{\mathrm{CH} 2}=11.9 \mathrm{~Hz}\right.$, $\left.1 \mathrm{H}, 0.5 \mathrm{xCH}_{2}{ }^{\mathrm{Bn}}\right), 4.37\left(\mathrm{~d}, J_{1,2}=7.9 \mathrm{~Hz}, 1 \mathrm{H}, \mathrm{H}-1^{1}\right), 4.33-4.13(\mathrm{~m}$, $\left.6 \mathrm{H}, \mathrm{H}-3^{1}, 2 \mathrm{xH}-4,1.5 \mathrm{xH}-6\right), 4.01$ (m, 3H, 1.5xH-6), 3.93 (dd, $J_{2,3}=$ 10.4, $\left.J_{3,4}=3.4 \mathrm{~Hz}, 1 \mathrm{H}, \mathrm{H}-3^{2}\right), 3.71\left(\mathrm{~d}, J_{3,4}=2.5 \mathrm{~Hz}, 1 \mathrm{H}, \mathrm{H}-4^{3}\right), 3.55$ - 3.46 (m, 2H, H-3 ${ }^{3}, \mathrm{H}-5^{3}$ ), 3.32 (s, $1 \mathrm{H}, \mathrm{H}-5$ ), 3.30 (s, $\left.1 \mathrm{H}, \mathrm{H}-5\right)$, 1.94 (s, 3H, $\mathrm{CH}_{3}{ }^{\mathrm{Ac}}$ ), 1.09 (s, 9H, 3xCH$\left.{ }_{3}^{\mathrm{Piv}}\right), 1.06$ (s, 9H, 3xCH${ }^{\mathrm{Piv}}$ ), 1.05 (s, 9H, $\left.3 \mathrm{xCH}_{3}{ }^{\mathrm{Piv}}\right) .{ }^{13} \mathrm{C}$ NMR $\left(101 \mathrm{MHz} \mathrm{CDCl}_{3}\right) \delta 180.4$, 176.11, 176.05, 170.4, 138.0, 137.8, 137.7, 137.3, 129.9-126.1 (20C), 100.4 (2C), 100.3, 99.9, 99.7, 76.2, 76.0, 75.9, 75.83, 75.79, 74.0, 73.7, 72.7, 72.6, 71.9, 71.8, 71.1, 70.0, 68.9, 68.8, 67.4, 67.1, 62.5, 39.1, 38.8, 38.7, 27.3 (3C), 27.3 (3C), 27.1 (3C) 20.9. HRMS (ESI-TOF) m/z: $\left[\mathrm{M}+\mathrm{NH}_{4}\right]^{+}$Calcd for $\mathrm{C}_{63} \mathrm{H}_{82} \mathrm{NO}_{20} 1172.5430$ Found 1172.5440 .

Benzyl 4,6-O-benzylidene-3-O-chloroacetyl-2-O-pivaloyl- $\beta$-Dgalactopyranosyl-( $\rightarrow 3$ )-4,6- $O$-benzylidene-2- $O$-pivaloyl- $\beta$-Dgalactopyranosyl-( $\rightarrow 3)$-6-O-acetyl-4-O-benzyl-2-O-pivaloyl$\beta$-D-galactopyranosyl-( $\rightarrow 3$ )-4,6- $O$-benzylidene-2- $O$-pivaloyl$\beta$-D-galactopyranosyl-( $\rightarrow 3)-4,6-O$-benzylidene-2- $O$-pivaloyl$\boldsymbol{\beta}$-D-galactopyranoside (24). To a $25 \mathrm{~mL}$ flame-dried flask was added 23 (1.2 g, $1.0 \mathrm{mmol})$ and the $\mathbf{1 5 b}(1.2 \mathrm{~g}, 1.4 \mathrm{mmol})$. The mixture was dried azeotropically with toluene $(2 \times 10 \mathrm{~mL})$ and subjected to vacuum overnight. It was then dissolved in dry $\mathrm{CH}_{2} \mathrm{Cl}_{2}(5$ $\mathrm{mL}$ ) and dry $\mathrm{MeCN}(5 \mathrm{~mL})$, cooled to $-30^{\circ} \mathrm{C}$, followed by addition of NIS (336 mg; $1.5 \mathrm{mmol}$ ) and TESOTf (27 mg; $0.1 \mathrm{mmol}$ ). The reaction mixture was stirred at $-30^{\circ} \mathrm{C}$ until TLC revealed full conversion of the donor $(2 \mathrm{~h})$. The solution was diluted with $\mathrm{CH}_{2} \mathrm{Cl}_{2}$ $(100 \mathrm{~mL})$ and washed with sat. aq. $\mathrm{NaS}_{2} \mathrm{O}_{3}(100 \mathrm{~mL})$ and sat. aq. $\mathrm{NaHCO}_{3}(100 \mathrm{~mL})$. The organic phase was dried over $\mathrm{MgSO}_{4}$, filtered and concentrated. The product was purified by flash chromatography (6:1 Tol/EtOAc) to afford $\mathbf{2 4}$ as an off-white solid. $\mathrm{R}_{\mathrm{f}} 0.62$ (2:1 Tol/EtOAc). Yield: $1.6 \mathrm{~g}(80 \%) .{ }^{1} \mathbf{H}$ NMR (400 MHz, $\left.\mathrm{CDCl}_{3}\right)$ $\delta 7.53-7.36(\mathrm{~m}, 8 \mathrm{H}), 7.34-7.07(\mathrm{~m}, 22 \mathrm{H}), 5.53\left(\mathrm{~s}, 1 \mathrm{H}, \mathrm{CH}^{\text {benzyli- }}\right.$ dene), 5.48 (s, $\left.1 \mathrm{H}, \mathrm{CH}^{\text {benzylidene }}\right), 5.46$ (s, $\left.1 \mathrm{H}, \mathrm{CH}^{\text {benzylidene }}\right), 5.44$ (s, $\left.1 \mathrm{H}, \mathrm{CH}^{\text {benzylidene }}\right), 5.45-5.23\left(\mathrm{~m}, 1 \mathrm{H}, \mathrm{H}-2^{1}, \mathrm{H}-2^{5}\right), 4.93-4.86(\mathrm{~m}$, $\left.2 \mathrm{H}, \mathrm{H}-1^{1 / 2 / 3 / 4 / 5}, \mathrm{H}^{-3^{5}}\right), 4.83\left(\mathrm{~m}, 2 \mathrm{H}, \mathrm{CH}_{2}{ }^{\mathrm{Bn}}\right), 4.66\left(\mathrm{~d}, J_{1,2}=8.0 \mathrm{~Hz}\right.$, $\left.1 \mathrm{H}, \mathrm{H}-1^{1 / 2 / 3 / 4 / 5}\right), 4.63\left(\mathrm{~d}, J_{1,2}=7.9 \mathrm{~Hz}, 1 \mathrm{H}, \mathrm{H}-1^{1 / 2 / 3 / 4 / 5}\right), 4.54$ (d, $J_{1,2}$ $\left.=8.0 \mathrm{~Hz}, 1 \mathrm{H}, \mathrm{H}-1^{1 / 2 / 3 / 4 / 5}\right), 4.49\left(\mathrm{~d}, J_{\mathrm{CH} 2}=12.0 \mathrm{~Hz}, 1 \mathrm{H}, 0.5 \mathrm{xCH}_{2}{ }^{\mathrm{Bn}}\right)$, $4.44\left(\mathrm{~d}, J_{\mathrm{CH} 2}=12.3 \mathrm{~Hz}, 1 \mathrm{H}, 0.5 \mathrm{xCH}_{2}{ }^{\mathrm{Bn}}\right), 4.36\left(\mathrm{~d}, J_{1,2}=7.9 \mathrm{~Hz}, 1 \mathrm{H}\right.$, $\left.\mathrm{H}-1^{1 / 2 / 3 / 4 / 5}\right), 4.33-3.87\left(\mathrm{~m}, 20 \mathrm{H}, \mathrm{H}-3^{1}, \mathrm{H}-3^{4}, \mathrm{H}-4^{1 / 2 / 4 / 5}, \mathrm{H}-4^{1 / 2 / 4 / 5}, \mathrm{H}-\right.$ $\left.4^{1 / 2 / 4 / 5}, \mathrm{H}-4^{1 / 2 / 4 / 5}, \mathrm{H}-6^{1}-\mathrm{H}-6^{5}, \mathrm{CH}_{2}{ }^{\mathrm{AcCl}}\right), 3.83-3.77$ (m, $\left.1 \mathrm{H}, \mathrm{H}-4^{3}\right)$, $3.43-3.38\left(\mathrm{~m}, 2 \mathrm{H}, \mathrm{H}-5^{3}, \mathrm{H}-5^{1 / 2 / 4 / 5}\right), 3.34-3.24\left(\mathrm{~m}, 3 \mathrm{H}, \mathrm{H}-5^{1 / 2 / 4 / 5}\right.$, $\left.\mathrm{H}-5^{1 / 2 / 4 / 5}, \mathrm{H}-5^{1 / 2 / 4 / 5}\right), 1.84$ (s, 3H, $\left.\mathrm{CH}_{3}{ }^{\mathrm{Ac}}\right), 1.10$ (s, $9 \mathrm{H}, 3 \mathrm{XCH}_{3}{ }^{\mathrm{Piv}}$ ), 1.07 (s, 9H, 3xCH$\left.{ }_{3}{ }^{\text {Piv }}\right), 1.04\left(\mathrm{~s}, 9 \mathrm{H}, 3 \mathrm{xCH}_{3}{ }^{\mathrm{Piv}}\right), 1.02(\mathrm{~s}, 9 \mathrm{H}$, $\left.3 \mathrm{xCH}_{3}{ }^{\mathrm{Piv}}\right), 1.00$ (s, 9H, $\left.3 \mathrm{xCH}_{3}{ }^{\mathrm{Piv}}\right) .{ }^{13} \mathbf{C}$ NMR $\left(101 \mathrm{MHz}, \mathrm{CDCl}_{3}\right) \delta$ 176.9 , 176.2, 176.0, 175.9, 175.8, 170.3, 167.1, 138.3, 137.9, 137.8, 137.7, 137.24, 137.15, 129.2-126.0 (30C), 100.9, 100.3 (2C), 100.2, 100.13, 100.09, 100.0, 99.9, 99.1, 77.3, 75.8, 75.6, 74.7, 74.4, 74.3, 73.5, 73.2, 73.0, 72.2, 71.81, 71.79, 71.76, 71.6, 71.4, 70.9, 70.0, 68.8, 68.7, 68.6, 68.2, 67.4, 66.9, 66.7, 62.0, 40.6, 38.8, 38.73, 38.66, 38.61, 38.57, 27.3 (3C), 27.23 (3C), 27.19 (3C), 27.1 (3C), 27.0 (3C), 20.8. HRMS (MALDI) m/z: [M + Na] ${ }^{+}$Calcd for $\mathrm{C}_{101} \mathrm{H}_{124} \mathrm{ClO}_{33} \mathrm{Na} 1922.7605$ Found 1922.7640 .

Benzyl 4,6-O-benzylidene-2-O-pivaloyl- $\beta$-D-galactopyranosyl$(1 \rightarrow 3)-4,6-O$-benzylidene-2-O-pivaloyl- $\beta$-D-galactopyranosyl- 
$(1 \rightarrow 3)$-6- $O$-acetyl-4- $O$-benzyl-2- $O$-pivaloyl- $\beta$-D-galactopyranosyl-( $1 \rightarrow 3)-4,6-O$-benzylidene-2- $O$-pivaloyl- $\beta$-D-galactopyranosyl-( $1 \rightarrow 3)-4,6$ - $O$-benzylidene-2- $O$-pivaloyl- $\beta$-D-galactopyranoside (25). Compound 24 (3.0 g; $1.6 \mathrm{mmol}$ ) was dissolved in 30 mL dry THF. Thiourea (481 mg; $6.3 \mathrm{mmol}$ ), Bu4NI (116 mg; 0.3 mmol) and $\mathrm{NaHCO}_{3}(584 \mathrm{mg} ; 6.9 \mathrm{mmol}$ ) was added and the reaction mixture was heated to $55^{\circ} \mathrm{C}$ for $12 \mathrm{~h}$. The mixture was filtered, concentrated and purified by flash chromatography (4:1 Tol/EtOAc) to give 25 as a colorless syrup. $R_{\mathrm{f}} 0.49$ (2:1 Tol/EtOAc). Yield: $2.4 \mathrm{~g}$ (84\%). IR (neat, $\mathrm{cm}^{-1}$ ): 3064.90, 2906.56, 2871.51, 1740.83, 1479.69, 1454.99, 1397.85, 1366.58, 1276.77, 1229.22, 1172.47, 1134.96, 1087.71, 1047.90, 1027.22. ${ }^{1} \mathbf{H}$ NMR $\left(400 \mathrm{MHz}, \mathrm{CDCl}_{3}\right) \delta 7.54-7.36(\mathrm{~m}, 8 \mathrm{H}, \mathrm{Ar}-\mathrm{H}), 7.33-7.02(\mathrm{~m}$, 22H, Ar-H), 5.52 (s, 1H, CH $\left.{ }^{\text {benzylidene }}\right), 5.49$ (s, 1H, $\left.\mathrm{CH}^{\text {benzylidene }}\right)$, $5.48\left(\mathrm{~s}, 1 \mathrm{H}, \mathrm{CH}^{\text {benzylidene }}\right), 5.46\left(\mathrm{~s}, 1 \mathrm{H}, \mathrm{CH}^{\text {benzylidene }}\right), 5.43\left(\mathrm{dd}, J_{2,3}=\right.$ $\left.10.3, J_{1,2}=7.9 \mathrm{~Hz}, 1 \mathrm{H}, \mathrm{H}-2^{1 / 2 / 4 / 5}\right), 5.37\left(\mathrm{dd}, J_{2,3}=10.2, J_{1,2}=7.9 \mathrm{~Hz}\right.$, $\left.1 \mathrm{H}, \mathrm{H}^{2} 2^{1 / 2 / 4 / 5}\right), 5.28\left(\mathrm{dd}, J_{2,3}=10.4, J_{1,2}=7.9 \mathrm{~Hz}, 2 \mathrm{H}, \mathrm{H}-2^{1 / 2 / 4 / 5}, \mathrm{H}-\right.$ $2^{1 / 2 / 4 / 5}$ ), 4.99 (dd, $J=10.1,8.0 \mathrm{~Hz}, 1 \mathrm{H}, \mathrm{H}-2^{3}$ ), 4.85 (d, $J_{C H 2}=11.9$ $\left.\mathrm{Hz}, 1 \mathrm{H}, 0.5 \mathrm{xCH}_{2}{ }^{\mathrm{Bn}}\right), 4.81\left(\mathrm{~d}, J_{\mathrm{CH} 2}=11.9 \mathrm{~Hz}, 1 \mathrm{H}, 0.5 \mathrm{xCH}_{2}{ }^{\mathrm{Bn}}\right), 4.80$ $\left(\mathrm{d}, J_{1,2}=8.0 \mathrm{~Hz}, 1 \mathrm{H}, \mathrm{H}-1^{3}\right), 4.66\left(\mathrm{~d}, J_{1,2}=8.0 \mathrm{~Hz}, 1 \mathrm{H}, \mathrm{H}-2^{1 / 2 / 4 / 5}\right)$, $4.65\left(\mathrm{~d}, J_{1,2}=7.9 \mathrm{~Hz}, 1 \mathrm{H}, \mathrm{H}-2^{1 / 2 / 4 / 5}\right), 4.54\left(\mathrm{~d}, J_{1,2}=8.0 \mathrm{~Hz}, 1 \mathrm{H}, \mathrm{H}-\right.$ $\left.2^{1 / 2 / 4 / 5}\right), 4.51\left(\mathrm{~d}, J=12.0 \mathrm{~Hz}, 1 \mathrm{H}, 0.5 \mathrm{xCH}_{2}{ }^{\mathrm{Bn}}\right), 4.44$ (d, $J_{C H 2}=11.9$ $\left.\mathrm{Hz}, 1 \mathrm{H}, 0.5 \mathrm{xCH}_{2}{ }^{\mathrm{Bn}}\right), 4.36\left(\mathrm{~d}, J_{1,2}=7.9 \mathrm{~Hz}, 1 \mathrm{H}, \mathrm{H}-2^{1 / 2 / 4 / 5}\right), 4.31-$ 3.88 (m, $18 \mathrm{H}, \mathrm{H}-3^{1 / 2 / 4 / 5}, \mathrm{H}-3^{1 / 2 / 4 / 5}, \mathrm{H}-3^{1 / 2 / 4 / 5}, \mathrm{H}-3^{1 / 2 / 4 / 5}, 4 \times \mathrm{H}-4, \mathrm{H}-6^{1}-$ H-6 ${ }^{5}$ ), 3.81 (dd, $\left.J_{3,4}=2.9, J_{4,5}=1.2 \mathrm{~Hz}, 1 \mathrm{H}, \mathrm{H}-4\right), 3.54$ (dd, $J_{2,3}=$ $10.1, J_{3,4}=3.7 \mathrm{~Hz}, 1 \mathrm{H}, \mathrm{H}-3^{3}$ ), 3.41 (dd, $J_{5,6 a}=7.4, J_{5,6 b}=5.9 \mathrm{~Hz}$, $1 \mathrm{H}, \mathrm{H}-5^{3}$ ), $3.36-3.32$ (m, $1 \mathrm{H}, \mathrm{H}-5^{1 / 2 / 4 / 5}$ ), $3.32-3.29$ (m, $1 \mathrm{H}, \mathrm{H}-$ $5^{1 / 2 / 4 / 5}$ ), 3.28 (m, $\left.2 \mathrm{H}, \mathrm{H}-5^{1 / 2 / 4 / 5}\right), 1.84$ (s, $3 \mathrm{H}, \mathrm{CH}_{3}{ }^{\mathrm{Ac}}$ ), 1.10 (s, $9 \mathrm{H}$, $3 \mathrm{xCH}_{3}{ }^{\mathrm{Piv}}$ ), 1.07 (s, 9H, 3xCH$\left.{ }_{3}{ }^{\mathrm{Piv}}\right), 1.06$ (s, 9H, $\left.3 \mathrm{xCH}_{3}{ }^{\mathrm{Piv}}\right), 1.04$ (s, 9H, $3 \mathrm{xCH}_{3}{ }^{\mathrm{Piv}}$ ), 1.03 (s, 89H, $3 \mathrm{xCH}_{3}{ }^{\mathrm{Piv}}$ ). ${ }^{13} \mathrm{C}$ NMR $(101 \mathrm{MHz}$, $\left.\mathrm{CDCl}_{3}\right) \delta 178.9,176.3,176.1,176.0,175.9,170.4,138.4,138.0$, 137.9, 137.8, 137.4, 137.3, 129.4-126.2 (30C), 101.4, 100.38, 100.36 (2C), 100.2 (2C), 100.1, 100.0, 99.3, 77.4, 76.0, 75.9, 75.7 (2C), 74.8, 74.6, 74.4, 73.1, 72.3, 72.2 (2C), 72.0, 71.92, 71.86, 71.7, 71.5, 71.0, 70.1, 68.9, 68.8, 68.7, 68.1, 67.5 (2C), 67.1, 67.0, 62.1, 39.0, 38.82, 38.76, 38.71, 38.69, 27.4 (3C), 27.34 (3C), 27.29 (3C), 27.2 (3C), 27.1 (3C), 20.9. HRMS (ESI-TOF) m/z: [M + Na] ${ }^{+}$Calcd for $\mathrm{C}_{99} \mathrm{H}_{122} \mathrm{NaO}_{32}$ 1845.7817; Found 1845.7756 .

Benzyl 4,6-O-benzylidene-2,3-O-dipivaloyl- $\beta$-D-galactopyranosyl--(1 $\rightarrow 3)-4,6$-O-benzylidene-2-O-pivaloyl- $\beta$-D- $(1 \rightarrow 3)-4,6$ $O$-benzylidene-2-O-pivaloyl- $\beta$-D-galactopyranosyl- $(1 \rightarrow 3)-4,6$ $O$-benzylidene-2-O-pivaloyl- $\beta$-D-galactopyranosyl- $(1 \rightarrow 3)-6-O$ acetyl-4- $O$-benzyl-2- $O$-pivaloyl- $\beta$-D-galactopyranosyl-( $1 \rightarrow 3)$ 4,6-O-benzylidene-2- $O$-pivaloyl- $\beta$-D-galactopyranosyl- $(1 \rightarrow 3)$ 4,6-O-benzylidene-2-O-pivaloyl- $\beta$-D-galactopyranoside (26). To a $25 \mathrm{~mL}$ flame-dried flask was added 25 (2.0 g, $1.1 \mathrm{mmol})$ and the 20 (1.2 g, $1.4 \mathrm{mmol}$ ). The mixture was dried azeotropically with toluene $(2 \times 15 \mathrm{~mL})$ and subjected to vacuum overnight. It was then dissolved in dry $\mathrm{CH}_{2} \mathrm{Cl}_{2}(7.5 \mathrm{~mL})$ and dry $\mathrm{MeCN}(7.5 \mathrm{~mL})$, cooled to $-30{ }^{\circ} \mathrm{C}$, followed by addition of NIS (329 mg; $1.5 \mathrm{mmol}$ ) and TESOTf (29 mg; $0.1 \mathrm{mmol}$ ). The reaction mixture was stirred at $-30{ }^{\circ} \mathrm{C}$ until TLC revealed full conversion of the donor $(2 \mathrm{~h})$. The solution was diluted with $\mathrm{CH}_{2} \mathrm{Cl}_{2}(150 \mathrm{~mL})$ and washed with sat. aq. $\mathrm{NaS}_{2} \mathrm{O}_{3}(150 \mathrm{~mL})$ and sat. aq. $\mathrm{NaHCO}_{3}(150 \mathrm{~mL})$. The organic phase was dried over $\mathrm{MgSO}_{4}$, filtered and concentrated. The product was purified by flash chromatography (9:1 Tol/EtOAc) to afford an off-white solid. $\mathrm{R}_{\mathrm{f}} 0.66$ (2:1 Tol/EtOAc). Yield: $2.2 \mathrm{~g}$ (78\%). IR (neat, $\mathrm{cm}^{-1}$ ): 3539.46, 3064.70, 2972.47, 2932.93, 2906.87, 2872.21, 1739.43, 1700.74, 1479.84, 1455.43, 1397.77, 1367.06, 1276.81, 1172.99, 1086.91, 1048.10, 1000.89. ${ }^{1} \mathbf{H}$ NMR (400 MHz, CDCl $) \delta 7.64-7.44(\mathrm{~m}, 12 \mathrm{H}, \mathrm{Ar}-\mathrm{H}), 7.44-7.13(\mathrm{~m}$, 28H, Ar-H), 5.61 (s, 1H, CH $\left.{ }^{\text {benzylidene }}\right), 5.59$ (s, $\left.1 \mathrm{H}, \mathrm{CH}^{\text {benzylidene }}\right)$, 5.57 (s, 2H, 2xCH $\left.{ }^{\text {benzylidene }}\right), 5.56$ (s, $\left.1 \mathrm{H}, \mathrm{CH}^{\text {benzylidene }}\right), 5.54$ (s, $1 \mathrm{H}$, $\mathrm{CH}^{\text {benzylidene }}$ ), 5.52 - 5.34 (m, 7H, $\left.\mathrm{H}-2^{1}-\mathrm{H}-2^{7}\right), 4.96-4.87$ (m, 3H, $\left.\mathrm{CH}_{2}{ }^{\mathrm{Bn}}, \mathrm{H}-1\right), 4.80-4.72\left(\mathrm{~m}, 4 \mathrm{H}, 3 \times \mathrm{H}-1, \mathrm{H}-3^{7}\right), 4.70$ (d, $J_{1,2}=7.9$ $\mathrm{Hz}, 1 \mathrm{H}, \mathrm{H}-1$ ), 4.64 (d, $J_{1,2}=7.9 \mathrm{~Hz}, 1 \mathrm{H}, \mathrm{H}-1$ ), 4.62 (d, $J_{\mathrm{CH} 2}=12.1$
$\mathrm{Hz}, 1 \mathrm{H}, 0.5 \mathrm{xCH}_{2}{ }^{\mathrm{Bn}}$ ), 4.55 (d, $\left.J_{\mathrm{CH} 2}=11.9 \mathrm{~Hz}, 1 \mathrm{H}, 0.5 \mathrm{xCH}_{2}{ }^{\mathrm{Bn}}\right), 4.46$ (d, $\left.J_{1,2}=7.9 \mathrm{~Hz}, 1 \mathrm{H}, \mathrm{H}-1\right), 4.40-4.01\left(\mathrm{~m}, 25 \mathrm{H}, \mathrm{H}-3^{1}-\mathrm{H}-3^{6}, \mathrm{H}-4^{1}\right.$, $\mathrm{H}-4^{2}, \mathrm{H}-4^{4}, \mathrm{H}-4^{5}, \mathrm{H}-4^{6}, \mathrm{H}-4^{7}, \mathrm{H}-6^{1}, \mathrm{H}-6^{2}, \mathrm{H}-6 \mathrm{a}^{3}, \mathrm{H}-6^{4}, \mathrm{H}-6^{5}, \mathrm{H}-6^{6}$, H-6 ${ }^{7}$ ), 4.00 (dd, $J_{6 a, 6 b}=10.8, J_{5,6 b}=6,6 \mathrm{~Hz}, 1 \mathrm{H}, \mathrm{H}-6 \mathrm{~b}^{3}$ ), 3.89 (d, $J_{3,4}$ $\left.=3.3 \mathrm{~Hz}, 1 \mathrm{H}, \mathrm{H}-4^{3}\right), 3.50$ (t, $\left.J_{5,6 a}=J_{5,6 b}=6.6 \mathrm{~Hz}, 1 \mathrm{H}, \mathrm{H}-5^{3}\right), 3.45$ (s, $1 \mathrm{H}, \mathrm{H}-5^{1 / 2 / 4 / 5 / 6 / 7}$ ), 3.41 (s, $1 \mathrm{H}, \mathrm{H}-5^{1 / 2 / 4 / 5 / 6 / 7}$ ), 3.38 (s, $1 \mathrm{H}, \mathrm{H}-$ $5^{1 / 2 / 4 / 5 / 6 / 7}$ ), 3.34 (s, 2H, H-51/2/4/5/6/7, H-51/2/4/5/6/7), 3.31 (s, $1 \mathrm{H}$, $\mathrm{H}-5^{1 / 2 / 4 / 5 / 6 / 7}$ ), 1.93 (s, $3 \mathrm{H}, \mathrm{CH}_{3}{ }^{\mathrm{Ac}}$ ), 1.19 (s, 9H, 3xCH$\left.{ }^{\mathrm{Piv}}\right), 1.17$ (s, $18 \mathrm{H}, 3 \mathrm{xCH}_{3}{ }^{\mathrm{Piv}}$ ), 1.13 (s, 9H, $\left.3 \mathrm{xCH}_{3}{ }^{\mathrm{Piv}}\right), 1.13$ (s, $\left.9 \mathrm{H}, 3 \mathrm{xCH}_{3}{ }^{\mathrm{Piv}}\right), 1.13$ (s, 9H, $3 \mathrm{xCH}_{3}{ }^{\mathrm{Piv}}$ ), 1.11 (s, 9H, 3xCH$\left.{ }_{3}^{\mathrm{Piv}}\right), 1.10$ (s, 9H, 3xCH${ }^{\mathrm{Piv}}$ ). ${ }^{13}$ C NMR (101 MHz, $\left.\mathrm{CDCl}_{3}\right) \delta 178.1,176.8,176.2,176.1,175.99$, 175.95, 175.93, 175.8, 170.2, 137.92, 137.88, 137.86, 137.8, 137.7, 137.5, 137.2, 136.4, 129.7-125.3 (40C), 100.4-99.2 (13C), 75.862.1 (35C), 38.93, 38.90, 38.72, 38.68, 38.65, 38.61, 38.60, 38.58, 27.3 (3C), 27.22 (3C), 27.19 (3C), 27.19 (3C), 27.16 (3C), 27.14 (3C), 27.05 (3C), 27.0 (3C), 20.8. HRMS (ESI-TOF) m/z: $[\mathrm{M}+2 \mathrm{Na}]^{2+}$ Calcd for $\mathrm{C}_{140} \mathrm{H}_{174} \mathrm{Na}_{2} \mathrm{O}_{45}$ 1311.0573; Found 1311.0536 .

Benzyl 4,6-O-benzylidene-2,3-O-dipivaloyl- $\beta$-D-galactopyranosyl--( $1 \rightarrow 3)-4,6-O$-benzylidene-2- $O$-pivaloyl- $\beta$-D-galactopyranosyl-( $1 \rightarrow 3)-4,6$ - $O$-benzylidene-2- $O$-pivaloyl- $\beta$-D-galactopyranosyl- $(1 \rightarrow 3)-4,6-O$-benzylidene-2- $O$-pivaloyl- $\beta$-D-galactopyranosyl-( $1 \rightarrow 3$ )-4-O-benzyl-2- $O$-pivaloyl- $\beta$-D-galactopyranosyl$(1 \rightarrow 3)-4,6-O$-benzylidene-2-O-pivaloyl- $\beta$-D-galactopyranosyl$(1 \rightarrow 3)-4,6-O$-benzylidene-2- $O$-pivaloyl- $\beta$-D-galactopyranoside (27). 26 (2 g; $0.8 \mathrm{mmol})$ was dissolved in dry $\mathrm{CH}_{2} \mathrm{Cl}_{2}(20 \mathrm{~mL})$ and cooled to $0{ }^{\circ} \mathrm{C}$. A $1 \mathrm{M} \mathrm{L}$-selectride solution in THF (3.1 mL) was added and the reaction was stirred at $0{ }^{\circ} \mathrm{C}$ until complete consumption of the starting material $(1 \mathrm{~h})$. The reaction mixture was poured into sat. aq. $\mathrm{NH}_{4} \mathrm{Cl}(100 \mathrm{~mL})$. The aqueous phase was extract with $\mathrm{CH}_{2} \mathrm{Cl}_{2}(100 \mathrm{~mL})$ and the combined organic phase was dried over $\mathrm{MgSO}_{4}$, filtered and concentrated (avoid concentrating to dryness since the borane salts can be explosive). The crude product was purified by flash chromatography to give an off-white solid. Yield: 1.6 g (79\%). IR (neat, $\mathrm{cm}^{-1}$ ): 3511.92, 2972.77, 2933.51, 2907.86, 2873.19, 1736.95, 1700.87, 1479.95, 1397.88, 1366.87, 1276.67, 1168.85, 1135.17, 1080.83, 1046.25. ${ }^{\mathbf{1}} \mathbf{H}$ NMR (400 MHz, $\left.\mathrm{CDCl}_{3}\right)$ $\delta 7.51-7.07$ (m, 40H, Ar-H), 5.50 (s, 1H, CH $\left.{ }^{\text {benzylidene }}\right), 5.48$ (s, $\left.1 \mathrm{H}, \mathrm{CH}^{\text {benzylidene }}\right), 5.47$ (s, 2H, 2xCH $\left.\mathrm{CH}^{\text {benzylidene }}\right), 5.45$ (s, $1 \mathrm{H}, \mathrm{CH}^{\text {benzyl- }}$

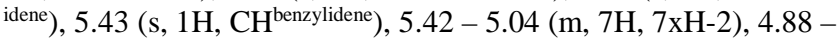
4.74 (m, 3H, H-1, $\mathrm{CH}_{2}{ }^{\mathrm{Bn}}$ ), $4.73-4.53$ (m, 4H, 4xH-1, H-37), 4.48 (d, $\left.J_{1,2}=7.7 \mathrm{~Hz}, 1 \mathrm{H}, \mathrm{H}-1\right), 4.44$ (d, $J_{\mathrm{CH} 2}=12.0 \mathrm{~Hz}, 1 \mathrm{H}, 0.5 \mathrm{xCH}_{2}{ }^{\mathrm{Bn}}$ ), 4.36 (d, $\left.J_{1,2}=7.9 \mathrm{~Hz}, 1 \mathrm{H}, \mathrm{H}-1\right), 4.31$ (d, $\left.J_{3,4}=4.0 \mathrm{~Hz}, 1 \mathrm{H}, \mathrm{H}-4\right)$, 4.29 - 3.80 (m, 23H, 6xH-3, 5xH-4, 6xH-6), 3.75 (d, $J_{3,4}=3.2 \mathrm{~Hz}$, $1 \mathrm{H}, \mathrm{H}-4), 3.52$ (dd, $\left.J_{6 a, 6 b}=11.3, J_{5,6 a}=5.8 \mathrm{~Hz}, 1 \mathrm{H}, \mathrm{H}-6 \mathrm{a}\right), 3.41-$ 3.35 (m, 1H, H-6b), 3.35 (s, 2H, 2xH-5), 3.31 (s, 1H, H-5), 3.27 (s, $1 \mathrm{H}, \mathrm{H}-5), 3.26$ - 3.24 (m, 2H, 2xH-5), 3.22 (s, 1H, H-5), 1.97 (s, $1 \mathrm{H},-\mathrm{OH}), 1.11$ (s, 9H, $3 \mathrm{xCH}_{3}{ }^{\mathrm{Piv}}$ ), 1.06 (s, 9H, $3 \mathrm{xCH}_{3}{ }^{\mathrm{Piv}}$ ), 1.05 (s, $9 \mathrm{H}, 3 \mathrm{xCH}_{3}{ }^{\mathrm{Piv}}$ ), 1.04 (s, 9H, 3xCH$\left.{ }_{3}^{\mathrm{Piv}}\right), 1.03$ (s, 9H, 3xCH$\left.{ }_{3}^{\mathrm{Piv}}\right), 1.03$ (s, $\left.9 \mathrm{H}, 3 \mathrm{xCH}_{3}{ }^{\mathrm{Piv}}\right), 1.00$ (s, $\left.9 \mathrm{H}, 3 \mathrm{xCH}_{3}{ }^{\mathrm{Piv}}\right), 0.98$ (s, 9H, $3 \mathrm{xCH}_{3}{ }^{\mathrm{Piv}}$ ). ${ }^{13}$ C NMR (101 MHz, $\left.\mathrm{CDCl}_{3}\right) \delta 178.3,176.9,176.3,176.23$, $176.20,176.08,176.06,176.0,138.1,138.0,137.90,137.87$, 137.86, 137.6, 137.3, 136.6, 129.9-125.4 (40C), 100.6-99.3 (13C), 75.9-66.9 (35C), 39.04, 39.03, 38.9, 38.81, 38.79, 38.74, 38.73, 38.69, 27.5 (3C), 27.4 (3C), 27.33 (3C), 27.32 (3C), 27.28 (3C), 27.24 (3C), 27.18 (3C), 27.12 (3C). HRMS (ESI-TOF) m/z: $[\mathrm{M}+2 \mathrm{Na}]^{2+}$ Calcd for $\mathrm{C}_{138} \mathrm{H}_{172} \mathrm{Na}_{2} \mathrm{O}_{44}$ 1290.0520; Found 1290.0459 .

Benzyl 4,6-O-benzylidene-3- $O$-chloroacetyl-2- $O$-pivaloyl- $\beta$-Dgalactopyranosyl- $(1 \rightarrow 3)-4,6-O$-benzylidene-2- $O$-pivaloyl- $\beta$-Dgalactopyranosyl- $(1 \rightarrow 3)-4,6-O$-benzylidene-2-O-pivaloyl- $\beta$-Dgalactopyranosyl- $(1 \rightarrow 3)-O-4,6-O$-benzylidene-2- $O$-pivaloyl- $\beta$ D-galactopyranoside (28). To a $50 \mathrm{~mL}$ flame-dried flask was added 16 (2.0 g, $2.57 \mathrm{mmol})$ and the $\mathbf{1 5 b}$ (2.6 g, $3.09 \mathrm{mmol})$. The 
mixture was dried azeotropically with toluene $(2 \times 30 \mathrm{~mL})$ and subjected to vacuum overnight. It was then dissolved in dry $\mathrm{CH}_{2} \mathrm{Cl}_{2}$ $(15 \mathrm{~mL})$ and dry $\mathrm{MeCN}(15 \mathrm{~mL})$, cooled to $-30{ }^{\circ} \mathrm{C}$, followed by addition of NIS (723 mg; $3.21 \mathrm{mmol}$ ) and TESOTf (68 mg; $0.26 \mathrm{mmol}$ ). The reaction mixture was stirred at $-30{ }^{\circ} \mathrm{C}$ until TLC revealed full conversion of the donor $\left(1 \frac{1}{2} \mathrm{~h}\right)$. The solution was diluted with $\mathrm{CH}_{2} \mathrm{Cl}_{2}(150 \mathrm{~mL})$ and washed with sat. aq. $\mathrm{NaS}_{2} \mathrm{O}_{3}$ $(150 \mathrm{~mL})$ and sat. aq. $\mathrm{NaHCO}_{3}(150 \mathrm{~mL})$. The organic phase was dried over $\mathrm{MgSO}_{4}$, filtered and concentrated. The product was purified by flash chromatography (9:1 toluene/EtOAc) to afford a white solid. $\mathrm{R}_{\mathrm{f}} 0.13$ (9:1 Tol/EtOAc). Yield: $3.1 \mathrm{~g}$ (88\%). ${ }^{1} \mathbf{H}$ NMR $\left(400 \mathrm{MHz}, \mathrm{CDCl}_{3}\right) \delta 7.50-7.36(\mathrm{~m}, 8 \mathrm{H}), 7.33-7.14(\mathrm{~m}, 17 \mathrm{H})$, 5.49 (s, $\left.1 \mathrm{H}, \mathrm{CH}^{\text {benzylidene }}\right), 5.48$ (s, $\left.1 \mathrm{H}, \mathrm{CH}^{\text {benzylidene }}\right), 5.46(\mathrm{~s}, 1 \mathrm{H}$, $\left.\mathrm{CH}^{\text {benzylidene }}\right), 5.42\left(\mathrm{~s}, 1 \mathrm{H}, \mathrm{CH}^{\text {benzylidene }}\right), 5.39$ (dd, $J_{2,3}=10.3, J_{3,4}=$ $\left.7.9 \mathrm{~Hz}, 1 \mathrm{H}, \mathrm{H}-2^{1 / 2 / 3 / 4}\right), 5.36-5.27$ (m, 3H, H-2 ${ }^{1 / 2 / 3 / 4}, \mathrm{H}-2^{1 / 2 / 3 / 4}, \mathrm{H}-$ $\left.2^{1 / 2 / 3 / 4}\right), 4.88\left(\mathrm{~d}, J_{1,2}=8.0 \mathrm{~Hz}, 1 \mathrm{H}, \mathrm{H}-1^{1 / 2 / 3 / 4}\right), 4.86\left(\mathrm{dd}, J_{2,3}=10.5\right.$, $\left.J_{3,4}=3.6 \mathrm{~Hz}, 1 \mathrm{H}, \mathrm{H}-3^{4}\right), 4.82\left(\mathrm{~d}, J_{\mathrm{CH} 2}=11.9 \mathrm{~Hz}, 1 \mathrm{H}, 0.5 \mathrm{xCH}_{2}{ }^{\mathrm{Bn}}\right)$, $4.68\left(\mathrm{~d}, J_{1,2}=7.9 \mathrm{~Hz}, 1 \mathrm{H}, \mathrm{H}-1^{1 / 2 / 3 / 4}\right), 4.67(\mathrm{~d}, J=7.9 \mathrm{~Hz}, 1 \mathrm{H}, \mathrm{H}-$ $\left.1^{1 / 2 / 3 / 4}\right), 4.44\left(\mathrm{~d}, J_{\mathrm{CH} 2}=11.9 \mathrm{~Hz}, 1 \mathrm{H}, 0.5 \mathrm{xCH}_{2}{ }^{\mathrm{Bn}}\right), 4.35$ (d, $J_{1,2}=7.9$ $\left.\mathrm{Hz}, 1 \mathrm{H}, \mathrm{H}-1^{1 / 2 / 3 / 4}\right), 4.30-3.84$ (m, $17 \mathrm{H}, \mathrm{H}-3^{1}-\mathrm{H}-3^{3}, \mathrm{H}-4^{1}-\mathrm{H}-4^{4}, \mathrm{H}-$ $\left.6^{1}-\mathrm{H}^{-} 6^{4}, \mathrm{CH}_{2}{ }^{\mathrm{AcCl}}\right), 3.35$ (m, $\left.1 \mathrm{H}, \mathrm{H}-5^{1 / 2 / 3 / 4}\right), 3.30\left(\mathrm{~m}, 1 \mathrm{H}, \mathrm{H}-5^{1 / 2 / 3 / 4}\right)$, $3.25\left(\mathrm{~m}, 1 \mathrm{H}, \mathrm{H}-5^{1 / 2 / 3 / 4}\right), 3.24\left(\mathrm{~m}, 1 \mathrm{H}, \mathrm{H}-5^{1 / 2 / 3 / 4}\right), 1.08$ (s, 9H, $3 \mathrm{xCH}_{3}{ }^{\mathrm{Piv}}$ ), 1.05 (s, 9H, 3xCH${ }_{3}^{\text {Piv }}$ ), 1.03 (s, 9H, 3xCH $3{ }^{\text {Piv }}$ ), 1.01 (s, $\left.9 \mathrm{H}, 3 \mathrm{xCH}_{3}{ }^{\mathrm{Piv}}\right) .{ }^{13} \mathrm{C}$ NMR $\left(101 \mathrm{MHz}, \mathrm{CDCl}_{3}\right) \delta 176.9,176.1$, 175.98, 175.96, 167.1, 137.9, 137.8, 137. 7, 137.3, 137.2, 129.2125.3 (25C), 100.9, 100.3 (2C), 100.1 (2C), 100.0, 99.7, 99.1, 75.9 (2C), 75.6, 73.5, 73.3, 72.1, 71.9, 71.5, 71.1, 70.8, 70.0, 69.8, 68.6, $68.5,68.3,67.41,67.36,66.9,66.6,40.6,38.8,38.7,38.6$ (2C), 27.3-21.5 (12C). HRMS (MALDI) m/z: [M + Na] ${ }^{+}$Calcd for $\mathrm{C}_{81} \mathrm{H}_{98} \mathrm{ClO}_{26} \mathrm{Na} 1544.5927$ Found 1544.5896.

Benzyl 4,6-O-benzylidene-2- $O$-pivaloyl- $\beta$-D-galactopyranosyl$(1 \rightarrow 3)-4,6-O$-benzylidene-2- $O$-pivaloyl- $\beta$-D-galactopyranosyl$(1 \rightarrow 3)-4,6-O$-benzylidene-2-O-pivaloyl- $\beta$-D-galactopyranosyl$(1 \rightarrow 3)$-4,6- $O$-benzylidene-2-pivaloyl- $\beta$-D-galactopyranoside (29). 28 (2.5 g; $1.6 \mathrm{mmol})$ was dissolved in dry $\mathrm{CH}_{2} \mathrm{Cl}_{2}(30 \mathrm{~mL})$ and cooled to $0{ }^{\circ} \mathrm{C}$. A $1 \mathrm{M} \mathrm{L}$-selectride solution in THF $(4.9 \mathrm{~mL})$ was added and the reaction was stirred at $0{ }^{\circ} \mathrm{C}$ until complete consumption of the starting material $(0.5 \mathrm{~h})$. The reaction mixture was poured into sat. aq. $\mathrm{NH}_{4} \mathrm{Cl}(100 \mathrm{~mL})$. The aqueous phase was extracted with $\mathrm{CH}_{2} \mathrm{Cl}_{2}(100 \mathrm{~mL})$. The combined organic phase was dried over $\mathrm{MgSO}_{4}$, filtered and concentrated (avoid concentrating to dryness since the borane salts can be explosive). The crude product was purified by flash chromatography ( $4: 1 \mathrm{Tol} / \mathrm{EtOAc})$ to give a white solid. $\mathrm{R}_{\mathrm{f}} 0.46$ (2:1 Tol/EtOAc). Yield: $2.2 \mathrm{~g}(94 \%) .{ }^{1} \mathbf{H}$ NMR (400 MHz, CDCl $) \delta 7.55-7.34(\mathrm{~m}, 8 \mathrm{H}), 7.34-7.11(\mathrm{~m}$, $17 \mathrm{H}), 5.53-5.44\left(\mathrm{~m}, 4 \mathrm{H}, 4 \mathrm{xCH} \mathrm{H}^{\text {benzylidene }}\right), 5.39$ (dd, $J_{2,3}=9.8, J_{1,2}$ $\left.=7.4 \mathrm{~Hz}, 1 \mathrm{H}, \mathrm{H}-2^{1 / 2 / 3 / 4}\right), 5.34\left(\mathrm{~m}, 2 \mathrm{H}, \mathrm{H}-2^{1 / 2 / 3 / 4}, \mathrm{H}-2^{1 / 2 / 3 / 4}\right), 4.97(\mathrm{dd}$, $\left.J_{2,3}=10.0, J_{1,2}=8.0 \mathrm{~Hz}, 1 \mathrm{H}, \mathrm{H}-2^{4}\right), 4.82\left(\mathrm{~d}, J_{\mathrm{CH} 2}=11.9 \mathrm{~Hz}, 1 \mathrm{H}\right.$, $\left.0.5 \mathrm{xCH}_{2}{ }^{\mathrm{Bn}}\right), 4.77\left(\mathrm{~d}, J_{1,2}=8.0 \mathrm{~Hz}, 1 \mathrm{H}, \mathrm{H}-1^{4}\right), 4.69\left(\mathrm{~d}, J_{1,2}=7.9 \mathrm{~Hz}\right.$, $\left.1 \mathrm{H}, \mathrm{H}-1^{1 / 2 / 3 / 4}\right), 4.68\left(\mathrm{~d}, J_{1,2}=8.0 \mathrm{~Hz}, 1 \mathrm{H}, \mathrm{H}-1^{1 / 2 / 3 / 4}\right), 4.44\left(\mathrm{~d}, J_{\mathrm{CH} 2}=\right.$ $\left.11.9 \mathrm{~Hz}, 1 \mathrm{H}, 0.5 \mathrm{xCH}_{2}{ }^{\mathrm{Bn}}\right), 4.36\left(\mathrm{~d}, J_{1,2}=7.8 \mathrm{~Hz}, 1 \mathrm{H}, \mathrm{H}-1^{1 / 2 / 3 / 4}\right), 4.31$ -3.90 (m, 15H, H-3 $\left.-3^{1}-3^{3}, \mathrm{H}-4^{1}-\mathrm{H}-4^{4}, \mathrm{H}-6^{1}-\mathrm{H}-6^{4}\right), 3.51$ (td, $J_{2,3}=$ 10.3, $\left.J_{3,4}=3.6 \mathrm{~Hz}, 1 \mathrm{H}, \mathrm{H}-3^{4}\right), 3.37-3.21\left(\mathrm{~m}, 4 \mathrm{H}, \mathrm{H}-5^{1}-\mathrm{H}-5^{4}\right), 1.07$ (s, 9H, $3 \mathrm{xCH}_{3}{ }^{\mathrm{Piv}}$ ), 1.06 (s, 9H, 3xCH${ }_{3}{ }^{\mathrm{Piv}}$ ), 1.05 (s, 9H, 3xCH${ }_{3}^{\mathrm{Piv}}$ ), 1.03 (s, 9H, 3xCH $3{ }^{\mathrm{Piv}}$ ). ${ }^{13} \mathrm{C}$ NMR $\left(101 \mathrm{MHz}, \mathrm{CDCl}_{3}\right) \delta$ 178.8, 176.1, 175.99, 175.97, 137.9, 137.8, 137.7, 137.3, 137.2, 129.3126.1 (25C), 101.3, 100.3 (2C), 100.3, 100.2, 100.0, 99.6, 99.2, 77.3, 76.0, 75.9, 75.6 (2C), 72.4, 72.1, 72.0, 71.9, 71.5, 71.1, 70.9, 70.0, 68.8, 68.7, 68.6, 67.41, 67.37, 66.93, 66.89, 38.9, 38.7, 38.6, 38.6, 27.23, 27.20, 27.16, 27.0. HRMS (MALDI) m/z: [M + Na] ${ }^{+}$ Calcd for $\mathrm{C}_{79} \mathrm{H}_{96} \mathrm{O}_{25} \mathrm{Na}$ 1467.6133 Found 1467.6114.

Benzyl 6-O-acetyl-4-O-benzyl-3-O-chloroacetyl-2-O-pivaloyl$\beta$-D-galactopyranosyl-( $\rightarrow 3$ )-4,6-O-benzylidene-2- $O$-pivaloyl$\beta$-D-galactopyranosyl-( $\rightarrow 3)-4,6$-O-benzylidene-2-O-pivaloyl$\beta$-D-galactopyranosyl-(1 $\rightarrow 3$ )-4,6-O-benzylidene-2-O-pivaloyl-
$\beta$-D-galactopyranosyl-(1 $\rightarrow 3$ )-4,6- $O$-benzylidene-2- $O$-pivaloyl及-D-galactopyranoside (30). To a $25 \mathrm{~mL}$ flame-dried flask was added 29 (2.8 g, $1.93 \mathrm{mmol})$ and the 21 (1.5 g, $2.7 \mathrm{mmol})$. The mixture was dried azeotropically with toluene $(2 \times 25 \mathrm{~mL})$ and subjected to vacuum overnight. It was then dissolved in dry $\mathrm{CH}_{2} \mathrm{Cl}_{2}$ $(12.5 \mathrm{~mL})$ and dry MeCN (12.5 mL), cooled to $-30^{\circ} \mathrm{C}$, followed by addition of NIS (651 mg; $2.90 \mathrm{mmol}$ ) and TESOTf (51 mg; $0.19 \mathrm{mmol}$ ). The reaction mixture was stirred at $-30^{\circ} \mathrm{C}$ until TLC revealed full conversion of the donor $(3 \mathrm{~h})$. The solution was diluted with $\mathrm{CH}_{2} \mathrm{Cl}_{2}(150 \mathrm{~mL})$ and washed with sat. aq. $\mathrm{NaS}_{2} \mathrm{O}_{3}$ $(150 \mathrm{~mL})$ and sat. aq. $\mathrm{NaHCO}_{3}(150 \mathrm{~mL})$. The organic phase was dried over MgSO4, filtered and concentrated. The product was purified by flash chromatography (4:1 Tol/EtOAc) to afford 30 as a white solid. $\mathrm{R}_{\mathrm{f}} 0.64$ (2:1 Tol/ EtOAc). Yield: $2.5 \mathrm{~g}(69 \%) .{ }^{1} \mathbf{H}$ NMR $\left(400 \mathrm{MHz}, \mathrm{CDCl}_{3}\right) \delta 7.50-7.37(\mathrm{~m}, 8 \mathrm{H}), 7.32-7.14(\mathrm{~m}, 17 \mathrm{H})$, $5.48\left(\mathrm{~s}, 1 \mathrm{H}, \mathrm{CH}^{\text {benzylidene }}\right), 5.47\left(\mathrm{~s}, 1 \mathrm{H}, \mathrm{CH}^{\text {benzylidene }}\right), 5.46(\mathrm{~s}, 2 \mathrm{H}$, $2 \mathrm{xCH} \mathrm{H}^{\text {benzylidene }}$ ), 5.39 (dd, $\left.J_{2,3}=10.2, J_{1,2}=7.9 \mathrm{~Hz}, 1 \mathrm{H}, \mathrm{H}-2^{1 / 2 / 3 / 4 / 5}\right)$, $5.33\left(\mathrm{dd}, J_{2,3}=10.5, J_{1,2}=8.0 \mathrm{~Hz}, 1 \mathrm{H}, \mathrm{H}-2^{1 / 2 / 3 / 4 / 5}\right), 5.30-5.23(\mathrm{~m}$, $\left.3 \mathrm{H}, \mathrm{H}-2^{1 / 2 / 3 / 4 / 5}, \mathrm{H}-2^{1 / 2 / 3 / 4 / 5}, \mathrm{H}-2^{1 / 2 / 3 / 4 / 5}\right), 4.87$ (dd, $J_{2,3}=10.2, J_{3,4}=$ $\left.3.1 \mathrm{~Hz}, 1 \mathrm{H}, \mathrm{H}-3^{5}\right), 4.82\left(\mathrm{~d}, J_{\mathrm{CH} 2}=11.9 \mathrm{~Hz}, 1 \mathrm{H}, 0.5 \mathrm{xCH}_{2}{ }^{\mathrm{Bn}}\right), 4.71$ (d, $\left.J_{1,2}=7.7 \mathrm{~Hz}, 1 \mathrm{H}, \mathrm{H}-1^{1 / 2 / 3 / 4 / 5}\right), 4.66(\mathrm{~m}, 3 \mathrm{H}, \mathrm{H}-11 / 2 / 3 / 4 / 5, \mathrm{H}-$ $11 / 2 / 3 / 4 / 5, \mathrm{H}-11 / 2 / 3 / 4 / 5), 4.62$ (d, $J_{\mathrm{CH} 2}=12.5 \mathrm{~Hz}, 1 \mathrm{H}, 0.5 \mathrm{xCH}_{2}{ }^{\mathrm{Bn}}$ ), $4.50\left(\mathrm{~d}, J_{\mathrm{CH} 2}=11.6 \mathrm{~Hz}, 1 \mathrm{H}, 0.5 \mathrm{xCH}_{2}{ }^{\mathrm{Bn}}\right), 4.44\left(\mathrm{~d}, J_{\mathrm{CH} 2}=11.9 \mathrm{~Hz}\right.$, $\left.1 \mathrm{H}, 0.5 \mathrm{xCH}_{2}{ }^{\mathrm{Bn}}\right), 4.36\left(\mathrm{~d}, J=7.9 \mathrm{~Hz}, 1 \mathrm{H}, \mathrm{H}-1^{1 / 2 / 3 / 4 / 5}\right), 4.30-3.90$ (m, $\left.18 \mathrm{H}, \mathrm{H}-3^{1}-\mathrm{H}-3^{4}, \mathrm{H}-4^{1}-\mathrm{H}-4^{4}, \mathrm{H}-6^{1}-\mathrm{H}-6^{5}\right), 3.84$ (d, $J_{3,4}=2.8 \mathrm{~Hz}$, $\left.1 \mathrm{H}, \mathrm{H}-4^{5}\right), 3.79\left(\mathrm{~d}, J_{\mathrm{CH} 2}=15.0 \mathrm{~Hz}, 1 \mathrm{H}, 0.5 \mathrm{xCH}_{2}{ }^{\mathrm{AcCl}}\right), 3.72\left(\mathrm{~d}, J_{\mathrm{CH} 2}\right.$ $\left.=15.0 \mathrm{~Hz}, 1 \mathrm{H}, 0.5 \mathrm{xCH}_{2}{ }^{\mathrm{AcCl}}\right), 3.60\left(\mathrm{t}, J_{5,6 a}=J_{5,6 b}=6.5 \mathrm{~Hz}, 1 \mathrm{H}, \mathrm{H}-\right.$ $5^{5}$ ), 3.31 (s, $\left.1 \mathrm{H}, \mathrm{H}-5^{1 / 2 / 3 / 4}\right), 3.27-3.23\left(\mathrm{~m}, 2 \mathrm{H}, \mathrm{H}-5^{1 / 2 / 3 / 4}\right), 3.21$ (s, $\left.1 \mathrm{H}, \mathrm{H}-5^{1 / 2 / 3 / 4}\right), 1.94$ (s, 3H, $\mathrm{CH}_{3}{ }^{\mathrm{Ac}}$ ), 1.07 (s, 9H, 3xCH${ }^{\mathrm{Piv}}$ ), 1.04 (s, 9H, 3xCH 3 Piv), 1.01 (s, 9H, $3 \mathrm{xCH}_{3}{ }^{\text {Piv }}$ ), 1.01 (s, 9H, 3xCH${ }^{\text {Piv }}$ ), 1.00 (s, 9H, 3xCH $\left.3{ }^{\mathrm{Piv}}\right) .{ }^{13} \mathrm{C}$ NMR (101 MHz, $\left.\mathrm{CDCl}_{3}\right) \delta 177.0,176.04$, 176.01, 175.97, 175.93, 170.2, 166.7, 137.9, 137.8, 137.73, 137.65, 137.19, 137.17, 129.0-126.0 (30C), 100.4, 100.34, 100.26, 100.2, 100.1, 100.0, 99.7, 99.6, 99.5, 77.3, 75.9, 75.8, 75.6, 75.1, 74.9, 73.6, 72.6, 71.9, 71.8, 71.5, 71.4, 71.2, 71.1, 71.0, 70.0, 68.9, 68.8, 68.7, 68.5, 67.5, 67.4, 67.3, 66.9, 62.1, 40.3, 38.8, 38.7, 38.63, 38.59 (2C), 27.21 (3C), 27.19 (6C), 27.1 (3C), 27.0 (3C), 20.8. HRMS (MALDI) m/z: $[\mathrm{M}+\mathrm{H}]{ }^{+}$Calcd for $\mathrm{C}_{101} \mathrm{H}_{125} \mathrm{ClO}_{33}$ 1900.7786 Found 1900.7671, $[\mathrm{M}+\mathrm{Na}]+$ Calcd for $\mathrm{C}_{101} \mathrm{H}_{124} \mathrm{ClO}_{33} \mathrm{Na} 1922.7605$ Found 1922.7614.

Benzyl 6-O-acetyl-4-O-benzyl-2- $O$-pivaloyl- $\beta$-D-galactopyranosyl-( $1 \rightarrow 3)$-4,6- $O$-benzylidene-2- $O$-pivaloyl- $\beta$-D-galactopyranosyl-( $\rightarrow 3$ )-4,6-O-benzylidene-2- $O$-pivaloyl- $\beta$-D-galactopyranosyl-( $1 \rightarrow 3)$-4,6- $O$-benzylidene-2- $O$-pivaloyl- $\beta$-D-galactopyranosyl-( $1 \rightarrow 3)-4,6$ - $O$-benzylidene-2- $O$-pivaloyl- $\beta$-D-galactopyranoside (31). 30 (2.0 g; $1.05 \mathrm{mmol}$ ) was dissolved in $30 \mathrm{~mL}$ dry THF. Thiourea (481 mg; $6.3 \mathrm{mmol}$ ), Bu $4 \mathrm{NI}$ (389 mg; $1.05 \mathrm{mmol}$ ) and $\mathrm{NaHCO}_{3}$ (265 mg; $3.15 \mathrm{mmol}$ ) was added and the reaction mixture was heated to $55^{\circ} \mathrm{C}$ for $12 \mathrm{~h}$. The mixture was filtered, concentrated and purified by flash chromatography (19:1 Tol/EtOAc) to give 31 as colorless syrup. $\mathrm{R}_{\mathrm{f}} 0.48$ (9:1 Tol/EtOAc). Yield: $1.6 \mathrm{~g}$ (83\%). IR (neat, $\mathrm{cm}^{-1}$ ): 3524.49, 3089.87, 2972.59, 2931.86, 2906.24, 2871.90, 1741.17, 1497.18, 1479.57, 1397.64, 1366.55, 1277.25, 1232.63, 1173.45, 1088.19, 1048.36, 1027.67. ${ }^{1} \mathbf{H}$ NMR (400 MHz, $\left.\mathrm{CDCl}_{3}\right) \delta 7.49-7.35$ (m, 12H), 7.32 - 7.13 (m, 18H), 5.48 (s, 1H, CH $\left.{ }^{\text {benzylidene }}\right), 5.47$ (s, 1H, $\left.\mathrm{CH}^{\text {benzylidene }}\right), 5.46$ (s, $\left.1 \mathrm{H}, \mathrm{CH}^{\text {benzylidene }}\right), 5.45$ (s, $\left.1 \mathrm{H}, \mathrm{CH}^{\text {benzylidene }}\right), 5.39$ (dd, $J_{2,3}=10.1$, $\left.J_{1,2}=8.1 \mathrm{~Hz}, 1 \mathrm{H}, \mathrm{H}-2\right), 5.36-5.26(\mathrm{~m}, 4 \mathrm{H}, 4 \mathrm{xH}-2), 4.85$ (d, $J_{\mathrm{CH} 2}=$ $\left.12.2 \mathrm{~Hz}, 1 \mathrm{H}, 0.5 \mathrm{xCH}_{2} \mathrm{Bn}\right), 4.83\left(\mathrm{~d}, J_{2,3}=10.1, J_{3,4}=3.2 \mathrm{~Hz}, 1 \mathrm{H}, \mathrm{H}-\right.$ $\left.2^{1}\right), 4.75\left(\mathrm{~d}, J_{\mathrm{CH} 2}=11.4 \mathrm{~Hz}, 1 \mathrm{H}, 0.5 \mathrm{xCH}_{2}{ }^{\mathrm{Bn}}\right), 4.69-4.59(\mathrm{~m}, 4 \mathrm{H}$, $\mathrm{H}-1^{2}-\mathrm{H}-1^{5}$ ), 4.61 (d, $J_{C H 2}=11.9 \mathrm{~Hz}, 1 \mathrm{H}, 0.5 \mathrm{xCH}_{2}{ }^{\mathrm{Bn}}$ ), 4.45 (d, $J_{C H 2}$ $\left.=11.9 \mathrm{~Hz}, 1 \mathrm{H}, 0.5 \mathrm{xCH}_{2}{ }^{\mathrm{Bn}}\right), 4.36\left(\mathrm{~d}, J 1,2=7.9 \mathrm{~Hz}, 1 \mathrm{H}, \mathrm{H}-1^{1}\right), 4.29$ - 3.93 (m, 18H, 4xH-3, H-41-H-4 $\left.{ }^{4}, 5 \times H-6\right), 3.89$ (dd, $J_{2,3}=10.3$, $J_{3,4}=3.2 \mathrm{~Hz}, 1 \mathrm{H}, \mathrm{H}-3$ ), 3.69 (d, $\left.J_{3,4}=2.7 \mathrm{~Hz}, 1 \mathrm{H}, \mathrm{H}-4^{5}\right), 3.49$ (t, $\left.J_{5,6 a}=J_{5,6 b}=6.8 \mathrm{~Hz}, 1 \mathrm{H}, \mathrm{H}-5^{5}\right), 3.31\left(\mathrm{~s}, 1 \mathrm{H}, \mathrm{H}-5^{1 / 2 / 3 / 4}\right), 3.26(\mathrm{~s}, 2 \mathrm{H}$, $\mathrm{H}-5^{1 / 2 / 3 / 4}, \mathrm{H}-5^{1 / 2 / 3 / 4}$ ), 3.22 (s, $1 \mathrm{H}, \mathrm{H}-5^{1 / 2 / 3 / 4}$ ), 1.94 (s, $3 \mathrm{H}, \mathrm{CH}_{3}{ }^{\mathrm{Ac}}$ ), 
1.07 (s, 9H, $\left.3 \mathrm{xCH}_{3}{ }^{\mathrm{Piv}}\right), 1.06$ (s, 9H, $\left.3 \mathrm{xCH}_{3}{ }^{\mathrm{Piv}}\right), 1.04$ (s, 9H, $3 \mathrm{xCH}_{3}{ }^{\mathrm{Piv}}$ ), 1.02 (s, 9H, 3xCH${ }^{\mathrm{Piv}}$ ), 1.01 (s, 9H, 3xCH$\left.{ }_{3}{ }^{\mathrm{Piv}}\right) .{ }^{13} \mathbf{C} \mathbf{N M R}$ $\left(101 \mathrm{MHz}, \mathrm{CDCl}_{3}\right) \delta 180.3,176.14,176.08$ (2C), 176.0, 170.4, 138.0, 137.87, 137.85, 137.73, 137.69, 137.3, 128.7-126.2 (30C), 100.5, 100.44 (2C), 100.41, 100.39, 100.2, 100.1, 99.8, 99.7, 99.6, 77.4, 76.2, 76.0, 75.9, 75.8, 75.7, 73.9, 73.6, 72.8, 72.7, 72.0, 71.9, 71.6, 71.3, 71.2, 71.1, 70.1, 68.9, 68.7, 68.6, 67.6, 67.5, 67.4, 67.0, 62.7, 39.1, 38.8, 38.73, 38.69, 38.66, 27.31 (3C), 27.28 (6C), 27.21 (3C), 27.0 (3C), 20.9 (3C). HRMS (MALDI) m/z: [M + Na] ${ }^{+}$Calcd for $\mathrm{C}_{99} \mathrm{H}_{122} \mathrm{O}_{32} \mathrm{Na} 1845.7811$ Found 1845.7806 .

Benzyl 4,6-O-benzylidene-2,3-di-O-pivaloyl- $\beta$-D-galactopyranosyl-(1 $\rightarrow 3)-4,6-O$-benzylidene-2- $O$-pivaloyl- $\beta$-D-galactopyranosyl-(1 $\rightarrow 3)-6-O$-acetyl-4-O-benzyl-2-O-pivaloyl- $\beta$-D-galactopyranosyl-( $\rightarrow 3$ )-4,6-O-benzylidene-2-O-pivaloyl- $\beta$-D-galactopyranosyl-( $\rightarrow 3)$-4,6-O-benzylidene-2- $O$-pivaloyl- $\beta$-D-galactopyranosyl-( $1 \rightarrow 3)-4,6$-benzylidene-2- $O$-pivaloyl- $\beta$-D-galactopyranosyl-( $1 \rightarrow 3)-4,6$ - $O$-benzylidene-2- $O$-pivaloyl- $\beta$-D-galactopyranoside (32). To a $25 \mathrm{~mL}$ flame-dried flask was added 31 (1.8 g, $0.99 \mathrm{mmol}$ ) and 20 (1.1 g, $1.3 \mathrm{mmol}$ ). The mixture was dried azeotropically with toluene (2x10 mL) and subjected to vacuum overnight. It was then dissolved in dry $\mathrm{CH}_{2} \mathrm{Cl}_{2}(5 \mathrm{~mL})$ and dry $\mathrm{MeCN}$ (5 mL), cooled to $-30{ }^{\circ} \mathrm{C}$, followed by addition of NIS (294 mg; $1.31 \mathrm{mmol}$ ) and TESOTf (26 mg; $0.1 \mathrm{mmol}$ ). The reaction mixture was stirred at $-30{ }^{\circ} \mathrm{C}$ until TLC revealed full conversion of the donor $(3 \mathrm{~h})$. The solution was diluted with $\mathrm{CH}_{2} \mathrm{Cl}_{2}(100 \mathrm{~mL})$ and washed with sat. aq. $\mathrm{NaS}_{2} \mathrm{O}_{3}(100 \mathrm{~mL})$ and sat. aq. $\mathrm{NaHCO}_{3}$ $(100 \mathrm{~mL})$. The organic phase was dried over $\mathrm{MgSO}_{4}$, filtered and concentrated. The product was purified by flash chromatography (19:1 Tol/EtOAc) to afford a white solid. $R_{f} 0.58$ (9:1 Tol/EtOAc). Yield: 1.95 g (77\%). IR (neat, $\left.\mathrm{cm}^{-1}\right)$ : 3065.85, 3035.79, 2972.47, 2871.63, 1738.98, 1479.57, 1454.98, 1397.69, 1366.40, 1276.70, 1172.84, 1132.25, 1087.66, 1047.67, 1027.15. ${ }^{1}$ H NMR $(400 \mathrm{MHz}$, $\left.\mathrm{CDCl}_{3}\right) \delta 7.61-6.91(\mathrm{~m}, 40 \mathrm{H}, \mathrm{Ar}-\mathrm{H}), 5.51$ (s, 1H, CH $\left.{ }^{\text {benzylidene }}\right)$, 5.48 (s, $\left.1 \mathrm{H}, \mathrm{CH}^{\text {benzylidene }}\right), 5.46$ (s, 2H, 2xCH $\left.{ }^{\text {benzylidene }}\right), 5.45$ (s, 2H, 2xCH $\left.{ }^{\text {benzylidene }}\right), 5.44-5.18(\mathrm{~m}, 7 \mathrm{H}, 7 \mathrm{xH}-2), 4.83(\mathrm{~d}, J=8.1 \mathrm{~Hz}$, $1 \mathrm{H}, \mathrm{H}-1), 4.83\left(\mathrm{~d}, J_{\mathrm{CH} 2}=11.7 \mathrm{~Hz}, 1 \mathrm{H}, 0.5 \mathrm{xCH}_{2}{ }^{\mathrm{Bn}}\right), 4.82$ (d, $J_{\mathrm{CH} 2}$ $\left.=11.9 \mathrm{~Hz}, 1 \mathrm{H}, 0.5 \mathrm{xCH}_{2}{ }^{\mathrm{Bn}}\right), 4.70\left(\mathrm{dd}, J_{2,3}=10.5, J_{3,4}=3.7 \mathrm{~Hz}, 1 \mathrm{H}\right.$, H-3 ${ }^{7}$ ), $4.68-4.57$ (m, 4H, 4xH-1), 4.52 (d, $J_{1,2}=7.7 \mathrm{~Hz}, 1 \mathrm{H}, \mathrm{H}-1$ ), $4.49\left(\mathrm{~d}, J_{\mathrm{CH} 2}=11.7 \mathrm{~Hz}, 1 \mathrm{H}, 0.5 \mathrm{xCH}_{2}{ }^{\mathrm{Bn}}\right), 4.44\left(\mathrm{~d}, J_{\mathrm{CH} 2}=11.9 \mathrm{~Hz}\right.$, $\left.1 \mathrm{H}, 0.5 \mathrm{xCH}_{2}{ }^{\mathrm{Bn}}\right), 4.35\left(\mathrm{~d}, J_{1,2}=7.9 \mathrm{~Hz}, 1 \mathrm{H}, \mathrm{H}-1\right), 4.33$ (d, $J_{3,4}=3.8$ $\mathrm{Hz}, 1 \mathrm{H}, \mathrm{H}-4), 4.27$ (d, $\left.J_{3,4}=3.3 \mathrm{~Hz}, 1 \mathrm{H}, \mathrm{H}-4\right), 4.26-3.93$ (m, $24 \mathrm{H}$, $6 \mathrm{xH}-3,5 \mathrm{xH}-4,6.5 \mathrm{xH}-6$ ), 3.90 (dd, $J_{6 a, 6 b}=11.0, J_{5,6 b}=7.1 \mathrm{~Hz}, 1 \mathrm{H}$, H-6b ${ }^{5}$ ), 3.78 (s, $1 \mathrm{H}, \mathrm{H}-5$ ), 3.41 (t, $J_{5,6 a}=J_{5,6 b}=6.6 \mathrm{~Hz}, 1 \mathrm{H}, \mathrm{H}-5^{5}$ ), 3.38 (s, 1H, H-5), 3.29 (s, 1H, H-5), 3.23 (m, 2H, 2xH-5), 3.21 (s, $1 \mathrm{H}, \mathrm{H}-5), 1.83$ (s, $3 \mathrm{H}_{,} \mathrm{CH}_{3}{ }^{\mathrm{Ac}}$ ), 1.10 (s, 9H, $3 \mathrm{xCH}_{3}{ }^{\mathrm{Piv}}$ ), 1.07 (s, $18 \mathrm{H}$, $6 \mathrm{xCH}_{3}{ }^{\mathrm{Piv}}$ ), 1.04 (s, 9H, 3xCH${ }_{3}{ }^{\mathrm{Piv}}$ ), 1.02 (s, 9H, 3xCH$\left.{ }_{3}{ }^{\mathrm{Piv}}\right), 1.00$ (s, $\left.18 \mathrm{H}, 6 \mathrm{xCH}_{3}{ }^{\mathrm{Piv}}\right), 0.99$ (s, 9H, $\left.3 \mathrm{xCH}_{3}{ }^{\mathrm{Piv}}\right) .{ }^{13} \mathrm{C}$ NMR $(101 \mathrm{MHz}$, $\left.\mathrm{CDCl}_{3}\right) \delta 178.2,177.0,176.4,176.2,176.15,176.06,176.0,175.9$, $170.4,138.4$, 138.0, 137.89, 137.86 (2C), 137.8, 137.6, 137.3, 129.1-126.0 (40C), 100.5, 100.4 (2C), 100.33, 100.30, 100.2 (2C), 100.13, 100.09, 100.0, 99.7, 99.2, 77.4, 76.0, 75.9, 75.8, 75.72, 75.71, 74.8, 74.5, 74.4, 73.1 (2C), 72.3, 71.99, 71.96, 71.9, 71.7, 71.6, 71.6, 71.5, 71.4, 71.3, 71.2, 71.1, 70.1, 68.8 (2C), 68.7, 68.6 (2C), 68.3, 67.6, 67.5 (2C), 67.0, 66.9, 62.3, 39.0, 38.81, 38.76 (2C), 38.71, 38.66, 38.65 (2C), 27.4 (3C), 27.29 (6C), 27.26 (3C), 27.24 (3C), 27.19 (3C), 27.1 (6C), 20.9. HRMS (MALDI) m/z: [M $+\mathrm{Na}]^{+}$Calcd for $\mathrm{C}_{140} \mathrm{H}_{174} \mathrm{O}_{45} \mathrm{Na}$ 2598.1219 Found 2598.1299.

\footnotetext{
Benzyl 4,6-O-benzylidene-2,3-O-dipivaloyl- $\beta$-D-galactopyranosyl--(1 $\rightarrow 3)-4,6-O$-benzylidene-2-O-pivaloyl- $\beta$-D-galactopyranosyl- $(1 \rightarrow 3)-6$ - $O$-acetyl-4-O-benzyl-3-O-chloroacetyl-2- $O$ pivaloyl- $\beta$-D-galactopyranosyl- $(1 \rightarrow 3)-4,6-O$-benzylidene-2- $O$ pivaloyl- $\beta$-D-galactopyranosyl- $(1 \rightarrow 3)-4,6$-O-benzylidene-2- $O$ pivaloyl- $\beta$-D-galactopyranosyl-( $1 \rightarrow 3)-4,6$ - $O$-benzylidene-2- $O$ pivaloyl- $\beta$-D-galactopyranosyl-( $\rightarrow 3$ )-4,6-O-benzylidene-2-O-
}

pivaloyl- $\beta$-D-galactopyranoside (33). 32 (2 g; $0.8 \mathrm{mmol}$ ) was dissolved in dry $\mathrm{CH}_{2} \mathrm{Cl}_{2}(20 \mathrm{~mL})$ and cooled to $0{ }^{\circ} \mathrm{C}$. A $1 \mathrm{M} \mathrm{L}$-selectride solution in THF ( $3.1 \mathrm{~mL})$ was added and the reaction was stirred at $0{ }^{\circ} \mathrm{C}$ until complete consumption of the starting material $(1 \mathrm{~h})$. The reaction mixture was poured into sat. aq. $\mathrm{NH}_{4} \mathrm{Cl}$ $(100 \mathrm{~mL})$. The aqueous phase was extract with $\mathrm{CH}_{2} \mathrm{Cl}_{2}(100 \mathrm{~mL})$ and the combined organic phase was dried over MgSO4, filtered and concentrated (avoid concentrating to dryness since the borane salts can be explosive). The crude product was purified by flash chromatography (9:1 Tol/EtOAc) to give $\mathbf{3 3}$ as a colorless solid material. Yield: $1.6 \mathrm{~g}(79 \%) .{ }^{1} \mathbf{H}$ NMR $\left(400 \mathrm{MHz}, \mathrm{CDCl}_{3}\right) \delta 7.52$ $7.02(\mathrm{~m}, 40 \mathrm{H}), 5.50$ (s, $\left.1 \mathrm{H}, \mathrm{CH}^{\text {benzylidene }}\right), 5.47$ (s, $\left.1 \mathrm{H}, \mathrm{CH}^{\text {benzylidene }}\right)$, 5.45 (m, 4H, 4xCH $\left.{ }^{\text {benzylidene }}\right), 5.43-5.16(\mathrm{~m}, 7 \mathrm{H}, 7 \mathrm{xH}-2), 4.84(\mathrm{~d}$, $\left.J_{1,2}=7.7 \mathrm{~Hz}, 1 \mathrm{H}, \mathrm{H}-1\right), 4.82\left(\mathrm{~d}, J_{\mathrm{CH} 2}=11.4 \mathrm{~Hz}, 1 \mathrm{H}, 0.5 \mathrm{xCH}_{2}{ }^{\mathrm{Bn}}\right)$, $4.79\left(\mathrm{~d}, J_{\mathrm{CH} 2}=11.4 \mathrm{~Hz}, 1 \mathrm{H}, 0.5 \mathrm{xCH}_{2}{ }^{\mathrm{Bn}}\right), 4.70\left(\mathrm{dd}, J_{2,3}=10.3, J_{3,4}=\right.$ $3.7 \mathrm{~Hz}, 1 \mathrm{H}, \mathrm{H}-3^{7}$ ), 4.67 (d, $\left.J_{1,2}=7.8 \mathrm{~Hz}, 1 \mathrm{H}, \mathrm{H}-1\right), 4.64$ (d, $J_{1,2}=$ $7.5 \mathrm{~Hz}, 1 \mathrm{H}, \mathrm{H}-1), 4.62$ (d, $\left.J_{1,2}=7.5 \mathrm{~Hz}, 1 \mathrm{H}, \mathrm{H}-1\right), 4.61$ (d, $J_{1,2}=7.7$ $\mathrm{Hz}, 1 \mathrm{H}, \mathrm{H}-1), 4.57$ (d, $\left.J_{1,2}=7.9 \mathrm{~Hz}, 1 \mathrm{H}, \mathrm{H}-1\right), 4.56$ (d, $J_{C H 2}=12.1$ $\left.\mathrm{Hz}, 1 \mathrm{H}, 0.5 \mathrm{xCH}_{2}{ }^{\mathrm{Bn}}\right), 4.45\left(\mathrm{~d}, J_{\mathrm{CH} 2}=12.0 \mathrm{~Hz}, 1 \mathrm{H}, 0.5 \mathrm{xCH}_{2}{ }^{\mathrm{Bn}}\right), 4.36$ (d, $\left.J_{1,2}=7.9 \mathrm{~Hz}, 1 \mathrm{H}, \mathrm{H}-1\right), 4.33$ (d, $\left.J_{3,4}=3.8 \mathrm{~Hz}, 1 \mathrm{H}, \mathrm{H}-4\right), 4.29-$ 3.88 (m, 23H, 6xH-3, 5xH-4, 6xH-6), 3.74 (d, J3,4 = 3.1 Hz, $1 \mathrm{H}, \mathrm{H}-$ 4), 3.52 (dd, $\left.J_{6 a, 6 b}=11.6, J_{5,6 a}=5.9 \mathrm{~Hz}, 1 \mathrm{H}, \mathrm{H}-6 \mathrm{a}^{5}\right), 3.39(\mathrm{~s}, 1 \mathrm{H}, \mathrm{H}-$ 5), $3.40-3.31\left(\mathrm{~m}, 1 \mathrm{H}, \mathrm{H}-6 \mathrm{~b}^{5}\right), 3.30$ (s, $\left.1 \mathrm{H}, \mathrm{H}-5\right), 3.24$ (s, $2 \mathrm{H}, 2 \mathrm{xH}-$ 5), 3.23 (s, 1H, H-5), 3.21 - 3.19 (m, 2H, 2xH-5), 2.08 (s, 1H, $\mathrm{OH}), 1.12$ (s, 9H, 3xCH$\left.{ }_{3}^{\mathrm{Piv}}\right), 1.07$ (s, $\left.18 \mathrm{H}, 6 \mathrm{xCH}_{3}{ }^{\mathrm{Piv}}\right), 1.04$ (s, 9H, $3 \mathrm{XCH}_{3}{ }^{\mathrm{Piv}}$ ), 1.03 (s, 9H, $3 \mathrm{xCH}_{3}{ }^{\mathrm{Piv}}$ ), 1.00 (s, 9H, $3 \mathrm{xCH}_{3}{ }^{\mathrm{Piv}}$ ), 0.98 (s, 9H, $\left.3 \mathrm{xCH}_{3}{ }^{\mathrm{Piv}}\right), 0.97$ (s, 9H, 3xCH$\left.{ }^{\mathrm{Piv}}\right) .{ }^{13} \mathrm{C}$ NMR $(101 \mathrm{MHz}$, $\left.\mathrm{CDCl}_{3}\right) \delta 178.2,177.0,176.4,176.3,176.1,176.1,176.0,176.0$ 138.3, 138.01, 137.99, 137.98, 137.91, 137.87, 137.6, 137.3, 129.9125.4 (40C), 100.6-99.2 (13C), 77.5, 76.2, 76.0, 75.9, 75.80, 75.78, 75.7, 75.0, 74.7, 74.2, 73.2, 72.5, 72.0, 71.9, 71.7, 71.6, 71.5, 71.4, $71.3,71.2,70.1,70.0,68.9,68.8,68.7,68.6,68.4,67.6,67.53$, 67.52, 67.49, 67.1, 66.9, 39.0, 38.9, 38.79, 38.78, 38.77, 38.74, 38.73, 38.69, 27.5 (3C), 27.4 (3C), 27.32 (3C), 27.29 (3C), 27.27 (3C), 27.25 (3C), 27.11 (3C), 27.10 (3C). HRMS (MALDI) m/z: $[\mathrm{M}+\mathrm{Na}]^{+}$Calcd for $\mathrm{C}_{99} \mathrm{H}_{122} \mathrm{O}_{32} \mathrm{Na} 1845.7811$ Found 1845.7806 .

Phenyl 2,3,5-tri-O-benzoyl-1-thio- $\alpha$-L-arabinofuranoside (34). To a solution of methyl 2,3,5-tri-O-benzoyl- $\alpha$-L-arabinofuranoside (7.8 g, $16.4 \mathrm{mmol})$ in $\mathrm{CH}_{2} \mathrm{Cl}_{2}(200 \mathrm{~mL})$ at $0{ }^{\circ} \mathrm{C}$ was added thiophenol $(2.4 \mathrm{~mL}, 22.4 \mathrm{mmol})$ dropwise. The reaction mixture was stirred at $0{ }^{\circ} \mathrm{C}$ for $15 \mathrm{~min}$, then $\mathrm{BF}_{3} \cdot \mathrm{Et}_{2} \mathrm{O}(13.6 \mathrm{~mL}, 107.2 \mathrm{mmol})$ was added and the resulting mixture was warmed gradually to $22{ }^{\circ} \mathrm{C}$. The reaction was stirred for $8 \mathrm{~h}$ at $22^{\circ} \mathrm{C}$. The reaction mixture was poured into sat. aq. $\mathrm{NaHCO}_{3}(100 \mathrm{~mL})$. The water phase was extracted with $\mathrm{CH}_{2} \mathrm{Cl}_{2}(50 \mathrm{~mL})$. The combined organic phases were dried over $\mathrm{MgSO}_{4}$, filtered and concentrated. The resulting residue was purified by column chromatography (20:1 Tol/EtOAc) to afford 34 as an amorphous solid. Rf 0.65 (9:1 Tol/EtOAc). Yield: $7.4 \mathrm{~g}$ (81\%). IR (neat, $\mathrm{cm}^{-1}$ ): 3061.69, 1721.80, 1601.55, 1584.07, 1480.24, 1451.45, 1440.37, 1315.28, 1266.25, 1177.61, 1107.62, 1095.64, 1069.33, 1026.52 ${ }^{1} \mathbf{H}$ NMR $\left(400 \mathrm{MHz}, \mathrm{CDCl}_{3}\right) \delta 8.06(\mathrm{~m}$, 2H, Ar-H), 8.03 - 7.91 (m, 4H, Ar-H), 7.62 - 7.14 (m, 14H, Ar-H), 5.77 (d, $J=3.7 \mathrm{~Hz}, 1 \mathrm{H}, \mathrm{H}-1), 5.70-5.63$ (m, 1H, H-2), 5.60 (s, 1H, H-3), 4.91 - 4.60 (m, 2H, H-4, H-5a, H-5b). ${ }^{13}$ C NMR (101 $\left.\mathrm{MHz} \mathrm{CDCl}_{3}\right) \delta 166.2,165.6,165.4,133.7-127.9$ (24C), 91.5, 82.6, 81.2, 78.1, 63.5. HRMS (ESI-TOF) $\mathrm{m} / \mathrm{z}:[\mathrm{M}+\mathrm{Na}]^{+}$Calcd for $\mathrm{C}_{138} \mathrm{H}_{172} \mathrm{O}_{44} \mathrm{Na} 2556.1113$ Found 2556.1149.

Benzyl 4,6- $O$-benzylidene-2,3-di- $O$-pivaloyl- $\beta$-D-galactopyranosyl-( $1 \rightarrow 3)$-4,6-O-benzylidene-2- $O$-pivaloyl- $\beta$-D-galactopyranosyl-( $1 \rightarrow 3)-4,6$ - $O$-benzylidene-2- $O$-pivaloyl- $\beta$-D-galactopyranosyl-( $1 \rightarrow 3)-4,6$ - $O$-benzylidene-2- $O$-pivaloyl- $\beta$-D-galactopyranosyl-( $1 \rightarrow 3)$-[2,3,5-tri- $O$-benzoyl- $\alpha$-L-arabinosyl- $(1 \rightarrow 6)]-4-O$ benzyl-2- $O$-pivaloyl- $\beta$-D-galactopyranosyl-( $1 \rightarrow 3)-4,6$ - $O$-benzylidene-2- $O$-pivaloyl- $\beta$-D-galactopyranosyl-( $1 \rightarrow 3)-4,6$ - $O$-benzylidene-2-O-pivaloyl- $\beta$-D-galactopyranoside (36). To a $25 \mathrm{~mL}$ 
flame-dried flask was added 27 (600 $\mathrm{mg}, 0.24 \mathrm{mmol}$ ) and the 34 (197 mg, $0.36 \mathrm{mmol}$ ). The mixture was dried azeotropically with toluene $(2 \times 5 \mathrm{~mL})$ and subjected to vacuum overnight. It was then dissolved in dry $\mathrm{CH}_{2} \mathrm{Cl}_{2}(2 \mathrm{~mL})$ and dry $\mathrm{MeCN}(2 \mathrm{~mL})$, cooled to $-30{ }^{\circ} \mathrm{C}$, followed by addition of NIS (82 $\mathrm{mg} ; 0.37 \mathrm{mmol}$ ) and TESOTf (13 mg; $0.05 \mathrm{mmol}$ ). The reaction mixture was stirred at $-30{ }^{\circ} \mathrm{C}$ until TLC revealed full conversion of the donor $(1 \mathrm{~h})$. The solution was diluted with $\mathrm{CH}_{2} \mathrm{Cl}_{2}(50 \mathrm{~mL})$ and washed with sat. aq. $\mathrm{NaS}_{2} \mathrm{O}_{3}(50 \mathrm{~mL})$ and sat. aq. $\mathrm{NaHCO}_{3}(50 \mathrm{~mL})$. The organic phase was dried over $\mathrm{MgSO}_{4}$, filtered and concentrated. The product was purified by flash chromatography (19:1 Tol/EtOAc) to afford a white solid. Yield: $486 \mathrm{mg}$ (69\%). IR (neat, $\mathrm{cm}^{-1}$ ): 3090.00, 3065.36, 2972.21, 2933.47, 2907.23, 2872.02, 1722.44, 1452.75, 1366.69, 1269.93, 1174.22, 1130.12, 1088.96, 1048.87, 1026.90 ${ }^{1} \mathbf{H}$ NMR (400 MHz, $\left.\mathrm{CDCl}_{3}\right) \delta 8.04$ (dd, $J=8.0,1.0 \mathrm{~Hz}, 2 \mathrm{H}, \mathrm{Ar}-$ $\mathrm{H}^{\mathrm{Bz}}$ ), 8.00 (dd, $J=8.2,1.3 \mathrm{~Hz}, 1 \mathrm{H}, \mathrm{Ar}-\mathrm{H}^{\mathrm{Bz}}$ ), 7.96 (dd, $J=8.1,1.2$ $\left.\mathrm{Hz}, 2 \mathrm{H}, \mathrm{Ar}-\mathrm{H}^{\mathrm{Bz}}\right), 7.60-7.46(\mathrm{~m}, 12 \mathrm{H}, \mathrm{Ar}-\mathrm{H}), 7.46-7.24(\mathrm{~m}, 31 \mathrm{H}$,

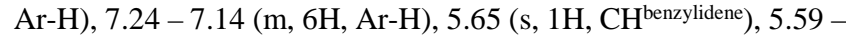
$5.56\left(\mathrm{~m}, 4 \mathrm{H}, 4 \mathrm{xCH}^{\text {benzylidene }}\right), 5.55$ (s, $\left.1 \mathrm{H}, \mathrm{CH}^{\text {benzylidene }}\right), 5.54-5.33$ (m, 9H, H-2 $\left.{ }^{1}-\mathrm{H}_{-2}{ }^{7}, \mathrm{H}-2^{\prime}, \mathrm{H}-3^{\prime}\right), 5.25$ (s, 1H, H-1'), 4.94 (d, $J_{1,2}=$ $7.9 \mathrm{~Hz}, 1 \mathrm{H}, \mathrm{H}-1), 4.92$ (d, $J_{\mathrm{CH} 2}=11.9 \mathrm{~Hz}, 1 \mathrm{H}, 0.5 \mathrm{xCH}_{2}{ }^{\mathrm{Bn}}$ ), 4.81 $\left(\mathrm{dd}, J_{2,3}=10.0, J_{3,4}=3.1 \mathrm{~Hz}, 1 \mathrm{H}, \mathrm{H}-3^{7}\right), 4.79\left(\mathrm{~d}, J_{\mathrm{CH} 2}=11.9 \mathrm{~Hz}\right.$, $1 \mathrm{H}, 0.5 \mathrm{xCH}_{2}{ }^{\mathrm{Bn}}$ ), $4.78-4.72(\mathrm{~m}, 4 \mathrm{H}, 4 \mathrm{xH}-1), 4.69\left(\mathrm{~d}, J_{1,2}=8.0 \mathrm{~Hz}\right.$, $1 \mathrm{H}, \mathrm{H}-1$ ), 4.64 (dd, $J_{5 a, 5 b}=12.1, J_{4,5 a}=3.4 \mathrm{~Hz}, 1 \mathrm{H}, \mathrm{H}-5 \mathrm{a}$ '), 4.55 (d, $\left.J_{\mathrm{CH} 2}=11.9 \mathrm{~Hz}, 1 \mathrm{H}, 0.5 \mathrm{xCH}_{2}{ }^{\mathrm{Bn}}\right), 4.48\left(\mathrm{dd} J_{5 a, 5 b}=12.1, J_{4,5 a}=4.7\right.$ $\left.\mathrm{Hz}, 1 \mathrm{H}, \mathrm{H}-5 \mathrm{~b}^{\prime}\right), 4.45-4.02$ (m, 27H, H-1, H-3 $-3^{1}-\mathrm{H}^{6}{ }^{6}, 6 \mathrm{xH}-$ $4^{1 / 2 / 4 / 5 / 6 / 7}, 6 \times \mathrm{H}^{-6 / 2 / 4 / 5 / 6 / 7}, \mathrm{H}-4$ ', $\left.0.5 \mathrm{xCH}_{2}{ }^{\mathrm{Bn}}\right), 3.94(\mathrm{~d}, J 3,4=3.7 \mathrm{~Hz}$, $1 \mathrm{H}, \mathrm{H}-4^{3}$ ), 3.92 (dd, $\left.J_{6 a, 6 b}=9.6, J_{5,6 a}=6.6 \mathrm{~Hz}, 1 \mathrm{H}, \mathrm{H}-6 \mathrm{a} 3\right), 3.66(\mathrm{t}$, $\left.J_{5,6 a}=J_{5,6 b}=6.6 \mathrm{~Hz}, 1 \mathrm{H}, \mathrm{H}-5^{3}\right), 3.56\left(\mathrm{dd}, J_{6 a, 6 b}=9.6, J_{5,6 b}=6.6 \mathrm{~Hz}\right.$, $\left.1 \mathrm{H}, \mathrm{H}-6 \mathrm{~b}^{3}\right), 3.50$ (s, $\left.1 \mathrm{H}, \mathrm{H}-4^{1 / 2 / 4 / 5 / 6 / 7}\right), 3.48$ (s, $\left.1 \mathrm{H}, \mathrm{H}-4^{1 / 2 / 4 / 5 / 6 / 7}\right), 3.41$ (s, $\left.1 \mathrm{H}, \mathrm{H}-4^{1 / 2 / 4 / 5 / 6 / 7}\right), 3.37$ (s, $\left.1 \mathrm{H}, \mathrm{H}-4^{1 / 2 / 4 / 5 / 6 / 7}\right), 3.35$ (s, $1 \mathrm{H}, \mathrm{H}^{-}$ $4^{1 / 2 / 4 / 5 / 6 / 7}$ ), 3.31 (s, $\left.1 \mathrm{H}, \mathrm{H}-4^{1 / 2 / 4 / 5 / 6 / 7}\right), 1.19$ (s, $\left.9 \mathrm{H}, 3 \mathrm{xCH}_{3}{ }^{\mathrm{Piv}}\right), 1.17$ (s, $\left.9 \mathrm{H}, 3 \mathrm{xCH}_{3}{ }^{\mathrm{Piv}}\right), 1.15$ (s, 9H, 3xCH$\left.{ }^{\mathrm{Piv}}\right), 1.15$ (s, $18 \mathrm{H}, 6 \mathrm{xCH}_{3}{ }^{\mathrm{Piv}}$ ), 1.10 (s, 18H, 6xCH $\left.3{ }^{\mathrm{Piv}}\right), 1.05$ (s, 9H, 3xCH $\left.3{ }^{\mathrm{Piv}}\right) .{ }^{13} \mathrm{C}$ NMR $(101 \mathrm{MHz}$, $\left.\mathrm{CDCl}_{3}\right) \delta 178.3,177.0,176.2,176.1$ (2C), 176.0, 175.94, 175.92, 166.3, 165.8, 165.5, 138.8, 137.98, 137.95 (2C), 137.9, 137.8, 137.6, 137.3, 134.1, 133.7, 133.2, 130.2-125.4 (55C), 106.4, 100.6, 100.5 (2C), 100.4, 100.3, 100.24 (2C), 100.21 (2C), 100.1, 99.84, 99.80, 99.4, 82.1, 81.5, 77.6, 77.4, 76.9, 76.1, 76.03, 76.99, 75.91, 75.86, 75.7, 74.5, 74.4, 74.2, 73.1, 72.4, 72.01, 71.96, 71.9, 71.6, 71.5, 71.3, 71.0, 70.1, 68.9, 68.8, 68.6, 68.4, 67.8, 67.6, 67.5, 67.4, 67.1, 67.0, 66.1, 63.6, 39.0, 38.83, 38.77, 38.73, 38.70 (2C), 38.68, 38.65, 27.4 (3C), 27.4 (3C), 27.32 (6C), 27.26 (3C), 27.2 (6C), 27.1 (3C).

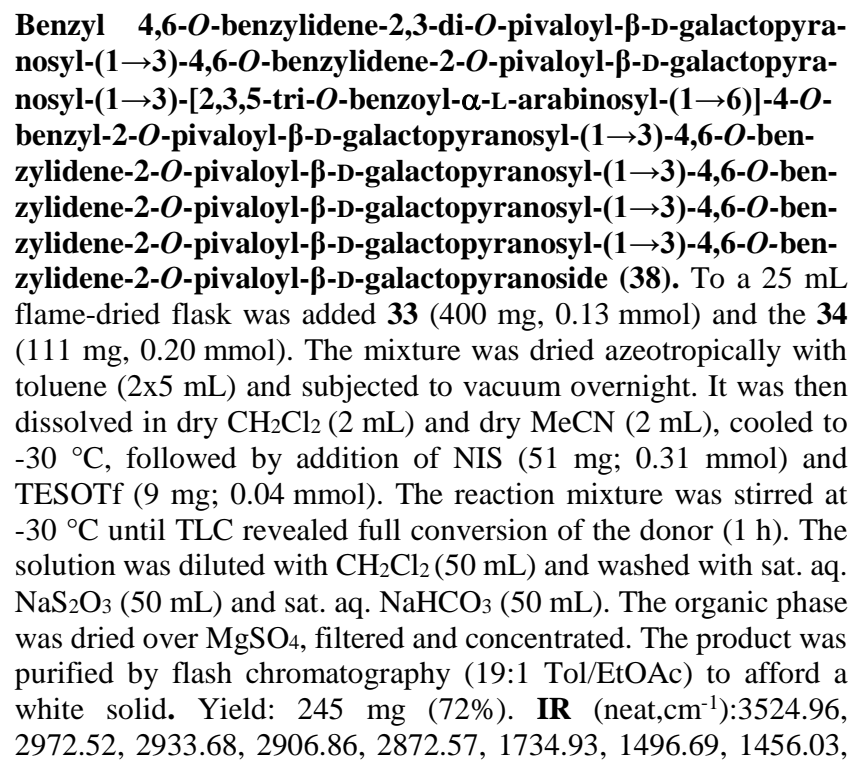
1397.62, 1366.61, 1277.07, 1170.07, 1082.44, 1046.46, 1001.32.
${ }^{1} \mathbf{H}$ NMR (400 MHz, $\left.\mathrm{CDCl}_{3}\right) \delta 8.11-7.81\left(\mathrm{~m}, 6 \mathrm{H}, \mathrm{Ar}-\mathrm{H}^{\mathrm{Bz}}\right), 7.58-$ 6.99 (m, 49H, Ar-H), 5.53 (s, 1H, CH $\left.{ }^{\text {benzylidene }}\right), 5.49$ (s, 1H, $\mathrm{CH}^{\text {ben- }}$ zylidene), 5.47 (s, $\left.1 \mathrm{H}, \mathrm{CH}^{\text {benzylidene }}\right), 5.47$ (s, $\left.1 \mathrm{H}, \mathrm{CH}^{\text {benzylidene }}\right), 5.45$ (s, $\left.1 \mathrm{H}, \mathrm{CH}^{\text {benzylidene }}\right), 5.44$ (s, $\left.1 \mathrm{H}, \mathrm{CH}^{\text {benzylidene }}\right), 5.43-5.42(\mathrm{~m}, 1 \mathrm{H}, \mathrm{H}-$ 2'/H-3',), $5.40-5.25$ (m, 8H, H-2'/H-3', H-2 $1-\mathrm{H}-2^{7}$ ), 5.14 (s, $1 \mathrm{H}$, H-1'), 4.83 (d, $\left.J_{1,2}=8.1 \mathrm{~Hz}, 1 \mathrm{H}, \mathrm{H}-1\right), 4.79$ (d, $J_{\mathrm{CH} 2}=11.9 \mathrm{~Hz}, 1 \mathrm{H}$, $\left.0.5 \mathrm{xCH}_{2}{ }^{\mathrm{Bn}}\right), 4.75-4.59$ (m, 8H, $5 \mathrm{xH}-1, \mathrm{H}-3^{7}, \mathrm{H}-5 \mathrm{a}$, $0.5 \mathrm{xCH}_{2}{ }^{\mathrm{Bn}}$ ), 4.50 (dd, $J_{5 a, 5 b}=12.3, J_{4,5 b}=4.6 \mathrm{~Hz}, 1 \mathrm{H}, \mathrm{H}-5 \mathrm{~b}$ '), 4.43 (d, $J_{C H 2}=$ $\left.11.9 \mathrm{~Hz}, 1 \mathrm{H}, 0.5 \mathrm{xCH}_{2}{ }^{\mathrm{Bn}}\right), 4.34\left(\mathrm{~d}, J_{1,2}=7.9 \mathrm{~Hz}, 1 \mathrm{H}, \mathrm{H}-1\right), 4.33-$ 3.90 (m, 26H, H-3 $3^{1}-\mathrm{H}-3^{6}, 6 x H-4^{1 / 2 / 3 / 4 / 6 / 7}, 6 x H-6^{1 / 2 / 3 / 4 / 6 / 7}, \mathrm{H}-4^{\prime}$, $0.5 \mathrm{xCH} 2^{\mathrm{Bn}}$ ), 3.84 (d, $J_{3,4}=2.9 \mathrm{~Hz}, 1 \mathrm{H}, \mathrm{H}-4^{5}$ ), 3.76 (dd, $J_{6 a, 6 b}=9.5$, $\left.J_{5,6 a}=6.0 \mathrm{~Hz}, 1 \mathrm{H}, \mathrm{H}-6 \mathrm{a}^{5}\right), 3.55\left(\mathrm{t}, J_{5,6 a}=J_{5,6 b}=6.0 \mathrm{~Hz}, 1 \mathrm{H}, \mathrm{H}-5^{5}\right)$, 3.48 (dd, $\left.J_{6 a, 6 b}=9.5, J_{5,6 b}=6.0 \mathrm{~Hz}, 1 \mathrm{H}, \mathrm{H}-6 \mathrm{~b}^{5}\right), 3.43(\mathrm{~s}, 1 \mathrm{H}, \mathrm{H}-$ $\left.5^{1 / 2 / 3 / 4 / 6 / 7}\right), 3.37$ (s, $\left.1 \mathrm{H}, \mathrm{H}-5^{1 / 2 / 3 / 4 / 6 / 7}\right), 3.30$ (s, $\left.1 \mathrm{H}, \mathrm{H}-5^{1 / 2 / 3 / 4 / 6 / 7}\right), 3.26$ (s, $1 \mathrm{H}, \mathrm{H}-5^{1 / 2 / 3 / 4 / 6 / 7}$ ), 3.23 (s, $1 \mathrm{H}, \mathrm{H}-5^{1 / 2 / 3 / 4 / 6 / 7}$ ), 3.22 (s, $1 \mathrm{H}, \mathrm{H}-$ $\left.5^{1 / 2 / 3 / 4 / 6 / 7}\right), 1.07$ (s, 9H, 3xCH $\left.{ }^{\mathrm{Piv}}\right), 1.07$ (s, 9H, 3xCH$\left.{ }_{3}^{\mathrm{Piv}}\right), 1.06$ (s, $\left.9 \mathrm{H}, 3 \mathrm{xCH}_{3}{ }^{\mathrm{Piv}}\right), 1.05$ (s, $\left.18 \mathrm{H}, 6 \mathrm{xCH}_{3}{ }^{\mathrm{Piv}}\right), 1.00$ (s, $\left.9 \mathrm{H}, 3 \mathrm{xCH}_{3}{ }^{\mathrm{Piv}}\right), 0.99$ (s, 9H, $\left.3 \mathrm{xCH}_{3}{ }^{\mathrm{Piv}}\right), 0.99$ (s, 9H, $\left.3 \mathrm{xCH}_{3}{ }^{\mathrm{Piv}}\right) .{ }^{13} \mathbf{C}$ NMR $(101 \mathrm{MHz}$, $\left.\mathrm{CDCl}_{3}\right) \delta 178.3,176.9,176.20,176.18,176.02,175.98,175.97$, $175.8,166.3,166.24,166.22,138.7,138.03,137.96,137.92$, 137.86, 137.8, 137.6, 137.3, 133.8, 133.7, 133.3, 130.2-125.4 (55C), 106.5, 101.1, 100.6, 100.5, 100.4, 100.34, 100.29 (2C), 100.2 (2C), 100.1, 100.0, 99.8, 99.3, 82.6, 82.1, 81.5, 81.4, 78.1, 77.6, 77.4, 76.9, 76.03, 75.95, 75.91, 75.7, 74.54, 74.46, 74.2, 73.2, 72.3, 72.0, 71.9, 71.74, 71.69, 71.6, 71.5, 71.4, 71.3, 71.1, 70.0, 68.9, 68.6, 68.4, 67.8, 67.7, 67.5, 67.3, 67.1, 66.9, 64.0, 63.8, 63.6, 39.0, 38.8, 38.73 (5C), 38.68, 27.4 (3C), 27.3 (6C), 27.3 (6C), 27.20 (3C), 27.16 (3C), 27.1 (3C).

Benzyl 4,6- $O$-benzylidene-2,3-di- $O$-pivaloyl- $\beta$-D-galactopyranosyl- $(1 \rightarrow 3)-4,6$ - $O$-benzylidene-2- $O$-pivaloyl- $\beta$-D-galactopyranosyl-( $\rightarrow 3)$-[4,6-O-benzylidene-2,3-di- $O$-pivaloyl- $\beta$-D-galactopyranosyl-( $1 \rightarrow 6)$-4-O-benzyl-2,3-di- $O$-pivaloyl- $\beta$-D-galactopyranosyl-( $1 \rightarrow 6)]-4-O$-benzyl-2-O-pivaloyl- $\beta$-D-galactopyranosyl-( $1 \rightarrow 3)-4,6-O$-benzylidene-2- $O$-pivaloyl- $\beta$-D-galactopyranosyl-( $1 \rightarrow 3)$-4,6- $O$-benzylidene-2- $O$-pivaloyl- $\beta$-D-galactopyranosyl-( $1 \rightarrow 3)-4,6-O$-benzylidene-2- $O$-pivaloyl- $\beta$-D-galactopyranosyl-( $\rightarrow 3)-4,6-O$-benzylidene-2- $O$-pivaloyl- $\beta$-D-galactopyranoside (40). To a $25 \mathrm{~mL}$ flame-dried flask was added 33 (500 mg, $0.20 \mathrm{mmol})$ and the $35(281 \mathrm{mg}, 0.30 \mathrm{mmol})$. The mixture was dried azeotropically with toluene $(2 \times 5 \mathrm{~mL})$ and subjected to vacuum overnight. It was then dissolved in dry $\mathrm{CH}_{2} \mathrm{Cl}_{2}(2 \mathrm{~mL})$ and dry MeCN (2 mL), cooled to $-30{ }^{\circ} \mathrm{C}$, followed by addition of NIS (69 mg; $0.31 \mathrm{mmol})$ and TESOTf (10 mg; $0.04 \mathrm{mmol})$. The reaction mixture was stirred at $-30{ }^{\circ} \mathrm{C}$ until TLC revealed full conversion of the donor $(1 \mathrm{~h})$. The solution was diluted with $\mathrm{CH}_{2} \mathrm{Cl}_{2}$ $(50 \mathrm{~mL})$ and washed with sat. aq. $\mathrm{NaS}_{2} \mathrm{O}_{3}(50 \mathrm{~mL})$ and sat. aq. $\mathrm{NaHCO}_{3}(50 \mathrm{~mL})$. The organic phase was dried over $\mathrm{MgSO}_{4}$, filtered and concentrated. The product was purified by flash chromatography (19:1 Tol/EtOAc) to afford a white solid. Yield: $505 \mathrm{mg}$ (76\%). IR (neat, $\mathrm{cm}^{-1}$ ): 3524.96, 2972.52, 2933.68, 2906.86, 2872.57, 1734.93, 1496.69, 1456.03, 1397.62, 1366.61 1277.07, 1170.07, 1082.44, 1046.46, 1001.32. ${ }^{\mathbf{1}} \mathbf{H}$ NMR (400 MHz, $\left.\mathrm{CDCl}_{3}\right)$ $\delta 7.41(\mathrm{~m}, 14 \mathrm{H}, \mathrm{Ar}-\mathrm{H}), 7.33-7.03(\mathrm{~m}, 38 \mathrm{H}, \mathrm{Ar}-\mathrm{H}), 5.53-5.11(\mathrm{~m}$, $16 \mathrm{H}, 7 \mathrm{XCH}^{\text {benzylidene, }} \mathrm{H}-2^{1}-\mathrm{H}-2^{7}, \mathrm{H}-2^{1}, \mathrm{H}-2^{2}$ ), 4.94 (dd, $J_{2,3}=10.4$, $\left.J_{3,4}=3.1 \mathrm{~Hz}, 1 \mathrm{H}, \mathrm{H}-3^{7 / 1^{\prime} / 2^{\prime}}\right), 4.84$ (d, $\left.J_{1,2}=7.9 \mathrm{~Hz}, 1 \mathrm{H}, \mathrm{H}-1\right), 4.89-$ $4.74\left(\mathrm{~m}, 3 \mathrm{H}, \mathrm{H}-3^{7 / 1^{\prime} / 2^{\prime}}, \mathrm{CH}_{2}{ }^{\mathrm{Bn}}\right), 4.73-4.60$ (m, 5H, 3xH-1, H$\left.3^{7 / 1^{\prime} / 2^{\prime}}, 0.5 \mathrm{xCH}_{2}{ }^{\mathrm{Bn}}\right), 4.59\left(\mathrm{~d}, J_{1,2}=8.1 \mathrm{~Hz}, 1 \mathrm{H}, \mathrm{H}-1\right), 4.55\left(\mathrm{~d}, J_{\mathrm{CH} 2}=\right.$ $11.2 \mathrm{~Hz}, 1 \mathrm{H}, 0.5 \mathrm{xCH}_{2}{ }^{\mathrm{Bn}}$ ), 4.47 (d, J1,2 = $7.7 \mathrm{~Hz}, 1 \mathrm{H}, \mathrm{H}-1$ ), 4.45 (d, $\left.J_{C H 2}=11.9 \mathrm{~Hz}, 1 \mathrm{H}, 0.5 \mathrm{xCH}_{2}{ }^{\mathrm{Bn}}\right), 4.41\left(\mathrm{~d}, J_{\mathrm{CH} 2}=12.2 \mathrm{~Hz}, 1 \mathrm{H}\right.$, $\left.0.5 \mathrm{xCH}_{2}{ }^{\mathrm{Bn}}\right), 4.36\left(\mathrm{~d}, J_{1,2}=7.9 \mathrm{~Hz}, 1 \mathrm{H}, \mathrm{H}-1\right), 4.32(\mathrm{~d}, J=3.8 \mathrm{~Hz}$, $\left.1 \mathrm{H}, \mathrm{H}-4^{1 / 2 / 3 / 4 / 6 / 7 / 2^{\prime}}\right), 4.28$ (d, $J=4.0 \mathrm{~Hz}, 1 \mathrm{H}, \mathrm{H}-4^{1 / 2 / 3 / 4 / 6 / 7 / 2}$ ), $4.25-$ $3.87\left(\mathrm{~m}, \quad 30 \mathrm{H}, \quad \mathrm{H}-3^{1}-\mathrm{H}-3^{6}, \mathrm{H}-4^{1 / 2 / 3 / 4 / 6 / 7 / 2}, \quad \mathrm{H}-4^{1 / 2 / 3 / 4 / 6 / 7 / 2}, \quad \mathrm{H}-\right.$ $4^{1 / 2 / 3 / 4 / 6 / 7 / 2}, \mathrm{H}-4^{1 / 2 / 3 / 4 / 6 / 7 / 2}, \mathrm{H}-4^{1 / 2 / 3 / 4 / 6 / 7 / 2}, \mathrm{H}-4^{1 / 2 / 3 / 4 / 6 / 7 / 2}, \mathrm{H}-6^{1}-\mathrm{H}-6^{7}$, H-6 $\left.{ }^{1}, \mathrm{H}-6^{2}{ }^{\prime}\right), 3.79$ (d, $\left.J_{3,4}=3.4 \mathrm{~Hz}, 1 \mathrm{H}, \mathrm{H}-4^{5 / 1^{\prime}}\right), 3.74$ (d, $J_{3,4}=3.8$ $\mathrm{Hz}, 1 \mathrm{H}, \mathrm{H}-4^{5 / 1^{\prime}}$ ), 3.52 (dd, $J_{5,6 a}=9.6, J_{5,6 b}=6.4 \mathrm{~Hz}, 1 \mathrm{H}, \mathrm{H}-5^{5 / 1^{\prime}}$ ), $3.48\left(\mathrm{dd}, J_{5,6 a}=7.9, J_{5,6 b}=5.6 \mathrm{~Hz}, 1 \mathrm{H}, \mathrm{H}-5^{5 / 1^{\prime}}\right), 3.39(\mathrm{~s}, 1 \mathrm{H}, \mathrm{H}-$ 
$\left.5^{1 / 2 / 3 / 4 / 6 / 7 / 2^{\prime}}\right), 3.35$ (s, $\left.1 \mathrm{H}, \mathrm{H}-5^{1 / 2 / 3 / 4 / 6 / 7 / 2^{\prime}}\right), 3.33$ (s, $\left.1 \mathrm{H}, \mathrm{H}-5^{1 / 2 / 3 / 4 / 6 / 7 / 2^{\prime}}\right)$, 3.31 (s, $\left.1 \mathrm{H}, \mathrm{H}-5^{1 / 2 / 3 / 4 / 6 / 7 / 2^{\prime}}\right), 3.29$ (s, $\left.1 \mathrm{H}, \mathrm{H}-5^{1 / 2 / 3 / 4 / 6 / 7 / 2^{\prime}}\right), 3.26$ (s, $1 \mathrm{H}$, $\left.\mathrm{H}-5^{1 / 2 / 3 / 4 / 6 / 7 / 2^{\prime}}\right), 3.20$ (s, $\left.1 \mathrm{H}, \mathrm{H}-5^{1 / 2 / 3 / 4 / 6 / 7 / 2^{\prime}}\right), 1.08$ (s, 9H, $3 \mathrm{XCH}_{3}{ }^{\mathrm{Piv}}$ ), 1.08 (s, 9H, $\left.3 \mathrm{xCH}_{3}{ }^{\mathrm{Piv}}\right), 1.075$ (s, $\left.18 \mathrm{H}, 3 \mathrm{xCH}_{3}{ }^{\mathrm{Piv}}\right), 1.07$ (s, 9H, $\left.3 \mathrm{xCH}_{3}{ }^{\text {Piv }}\right), 1.04$ (s, $\left.18 \mathrm{H}, 3 \mathrm{xCH}_{3}{ }^{\mathrm{Piv}}\right), 1.02\left(\mathrm{~s}, 9 \mathrm{H}, 3 \mathrm{xCH}_{3}{ }^{\mathrm{Piv}}\right), 1.02(\mathrm{~s}$, $\left.9 \mathrm{H}, 3 \mathrm{xCH}_{3}{ }^{\mathrm{Piv}}\right), 0.99$ (s, 9H, $\left.3 \mathrm{xCH}_{3}{ }^{\text {Piv }}\right), 0.98$ (s, 9H, 3xCH$\left.{ }_{3}{ }^{\mathrm{Piv}}\right), 0.98$ (s, 9H, $\left.3 \mathrm{xCH}_{3}{ }^{\mathrm{Piv}}\right) .{ }^{13} \mathrm{C}$ NMR $\left(101 \mathrm{MHz}, \mathrm{CDCl}_{3}\right) \delta 178.2,178.1$, 177.5, 176.8, 176.4, 176.3, 176.2, 176.1, 176.0, 175.89, 175.87, 175.8, 139.1, 138.1, 137.9, 137.9, 137.9, 137.82, 137.77, 137.6, 137.5, 137.2, 129.4-125.9 (38C), 101.7, 100.8, 100.5 (2C), 100.4, 100.34, 100.28, 100.2, 100.2 (2C), 100.13, 100.07, 100.0, 99.7, 99.6, 99.1, 77.3, 75.9, 75.93, 75.87, 75.70, 75.66, 75.3, 74.5, 74.31, 74.25, 73.2, 73.1, 72.3, 71.9, 71.8, 71.7, 71.5, 71.3, 71.2, 71.1, 70.9, $70.0,69.8,68.8,68.7,68.6,68.5,68.3,68.2,67.4,67.3,66.9,66.8$, 66.4, 66.3, 38.91, 38.91, 38.87, 38.74, 38.71, 38.67 (2C), 38.63, 38.61, 38.59, 38.58, 38.5, 27.29 (6C), 27.25 (3C), 27.23 (3C), 27.21 (3C), 27.19 (3C), 27.16 (3C), 27.14 (3C), 27.11 (3C), 27.0 (6C), 27.0 (3C).

Benzyl 4,6-O-benzylidene-2,3-di-O-pivaloyl- $\beta$-D-galactopyranosyl-( $1 \rightarrow 3)-4,6$ - $O$-benzylidene-2- $O$-pivaloyl- $\beta$-D-galactopyranosyl-( $1 \rightarrow 3)-4,6$ - $O$-benzylidene-2- $O$-pivaloyl- $\beta$ - $D$-galactopyranosyl-( $1 \rightarrow 3)-4,6$ - $O$-benzylidene-2- $O$-pivaloyl- $\beta$-D-galactopyranosyl- $(1 \rightarrow 3)$-[4,6- $O$-benzylidene-2,3-di- $O$-pivaloyl- $\beta$-D-galactopyranosyl-( $1 \rightarrow 3)-4,6-O$-benzylidene-2-O-pivaloyl- $\beta$-D-galactopyranosyl- $(1 \rightarrow 6)]-4-O$-benzyl-2- $O$-pivaloyl- $\beta$-D-galactopyranosyl-( $1 \rightarrow 3)-4,6$ - $O$-benzylidene-2- $O$-pivaloyl- $\beta$-D-galactopyranosyl-( $\rightarrow 3$ )-4,6- $O$-benzylidene-2- $O$-pivaloyl- $\beta$-D-galactopyranoside (37). To a $25 \mathrm{~mL}$ flame-dried flask was added 27 (600 mg, $0.24 \mathrm{mmol}$ ) and the 20 (306 $\mathrm{mg}, 0.36 \mathrm{mmol}$ ). The mixture was dried azeotropically with toluene (2x5 mL) and subjected to vacuum overnight. It was then dissolved in dry $\mathrm{CH}_{2} \mathrm{Cl}_{2}(2 \mathrm{~mL})$ and dry MeCN ( $2 \mathrm{~mL}$ ), cooled to $-30{ }^{\circ} \mathrm{C}$, followed by addition of NIS (82 $\mathrm{mg} ; 0.37 \mathrm{mmol})$ and TESOTf $(13 \mathrm{mg} ; 0.05 \mathrm{mmol})$. The reaction mixture was stirred at $-30{ }^{\circ} \mathrm{C}$ until TLC revealed full conversion of the donor $(2 \mathrm{~h})$. The solution was diluted with $\mathrm{CH}_{2} \mathrm{Cl}_{2}$ ( $50 \mathrm{~mL}$ ) and washed with sat. aq. $\mathrm{NaS}_{2} \mathrm{O}_{3}(50 \mathrm{~mL})$ and sat. aq. Na$\mathrm{HCO}_{3}(50 \mathrm{~mL})$. The organic phase was dried over $\mathrm{MgSO}_{4}$, filtered and concentrated. The product was purified by flash chromatography (19:1 Tol/EtOAc) to afford a white solid. Yield: $575 \mathrm{mg}$ (74\%). IR (neat, $\mathrm{cm}^{-1}$ ): 3535.58, 2972.41, 2872.16, 1736.19, 1700.99, 1479.69, 1455.60, 1397.63, 1366.51, 1276.86, 1171.43, 1132.66, 1084.76, 1046.59, 999.84. ${ }^{\mathbf{1}} \mathbf{H}$ NMR (400 MHz, $\left.\mathrm{CDCl}_{3}\right) \delta$ 7.43 (m, 16H, Ar-H), $7.34-7.14$ (m, 34H, Ar-H), 5.49 (s, 1H, $\left.\mathrm{CH}^{\text {benzylidene }}\right), 5.48-5.46\left(\mathrm{~m}, 2 \mathrm{H}, 2 \mathrm{xCH}^{\text {benzylidene }}\right), 5.45$ (s, 2H, $\left.2 \mathrm{xCH}^{\text {benzylidene }}\right), 5.44$ (s, 2H, 2xCH $\left.{ }^{\text {benzylidene }}\right), 5.42$ (s, $1 \mathrm{H}, \mathrm{CH}^{\text {benzyli- }}$ dene), $5.40-5.25$ (m, 7H, 7xH-2), 5.21 (dd, $J_{2,3}=10.3, J_{1,2}=7.9 \mathrm{~Hz}$, $1 \mathrm{H}, \mathrm{H}-2$ ), 5.13 (dd, $\left.J_{2,3}=10.3, J_{1,2}=8.0 \mathrm{~Hz}, 1 \mathrm{H}, \mathrm{H}-2\right), 4.85-4.76$ (m, $\left.4 \mathrm{H}, 2 \times \mathrm{H}-1, \mathrm{CH}_{2}{ }^{\mathrm{Bn}}\right), 4.74-4.62$ (m, 5H, 3xH-1, H-37, $\mathrm{H}-3^{2}$ ), $4.55\left(\mathrm{~d}, J_{1,2}=8.0 \mathrm{~Hz}, 1 \mathrm{H}, \mathrm{H}-1\right), 4.50\left(\mathrm{~d}, J_{\mathrm{CH} 2}=12.5 \mathrm{~Hz}, 1 \mathrm{H}\right.$, $\left.0.5 \mathrm{xCH}_{2}{ }^{\mathrm{Bn}}\right), 4.49\left(\mathrm{~d}, J_{1,2}=7.6 \mathrm{~Hz}, 1 \mathrm{H}, \mathrm{H}-1\right), 4.44\left(\mathrm{~d}, J_{\mathrm{CH} 2}=12.0\right.$ $\mathrm{Hz}, 1 \mathrm{H}, 0.5 \mathrm{xCH}_{2}{ }^{\mathrm{Bn}}$ ), 4.34 (d, $\left.J=8.0 \mathrm{~Hz}, 1 \mathrm{H}, \mathrm{H}-1\right), 4.33-3.91$ (m, $\left.29 \mathrm{H}, \mathrm{H}-3^{1}-\mathrm{H}-3^{6}, \mathrm{H}-3^{1}{ }^{\prime}, 8 \times \mathrm{H}-4^{1 / 2 / 4 / 5 / 6 / 7 / 1^{\prime} / 2^{2}}, 7.5 \times \mathrm{xH}-6^{1 / 2 / 4 / 5 / 6 / 7 / 1^{\prime} / 2^{\prime}}\right)$, $3.89\left(\mathrm{~d}, J_{1,2}=7.8 \mathrm{~Hz}, 1 \mathrm{H}, \mathrm{H}-1^{1}\right.$ ), $3.84\left(\mathrm{~d}, J_{6 a, 6 b}=11.9 \mathrm{~Hz}, 1 \mathrm{H}, \mathrm{H}-\right.$ $\left.6^{1 / 2 / 4 / 5 / 6 / 7 / 11^{\prime} / 2^{\prime}}\right), 3.74\left(\mathrm{~d}, J_{3,4}=3.4 \mathrm{~Hz}, 1 \mathrm{H}, \mathrm{H}-4^{3}\right), 3.64-3.52(\mathrm{~m}, 1 \mathrm{H}$, H-6 ${ }^{3}$ ), 3.47 (t, $\left.J_{5,6 a}=J_{5,6 b}=5.6 \mathrm{~Hz}, 1 \mathrm{H}, \mathrm{H}-5^{3}\right), 3.38(\mathrm{~s}, 1 \mathrm{H}, \mathrm{H}-$ $\left.5^{1 / 2 / 4 / 5 / 6 / 7 / 1^{\prime} / 2^{\prime}}\right), 3.35$ (s, $\left.1 \mathrm{H}, \mathrm{H}-5^{1 / 2 / 4 / 5 / 6 / 7 / 1^{\prime} / 2^{\prime}}\right), 3.30$ (s, $1 \mathrm{H}, \mathrm{H}-$ $\left.5^{1 / 2 / 4 / 5 / 6 / 7 / 1^{\prime} / 2^{2}}\right), 3.27$ (s, $\left.1 \mathrm{H}, \mathrm{H}-5^{1 / 2 / 4 / 5 / 6 / 7 / 1^{\prime} / 2^{\prime}}\right), 3.22$ (s, $1 \mathrm{H}, \mathrm{H}-$ $\left.5^{1 / 2 / 4 / 5 / 6 / 7 / 1^{\prime} / 2^{2}}\right), 3.20\left(\mathrm{~s}, 2 \mathrm{H}, 2 \mathrm{xH}-5^{1 / 2 / 4 / 5 / 6 / 7 / 1^{\prime} / 2^{2}}\right), 3.04$ (s, $1 \mathrm{H}, \mathrm{H}-$ $\left.5^{1 / 2 / 4 / 5 / 6 / 7 / 1^{\prime} / 2^{\prime}}\right), 1.13$ (s, 9H, $\left.3 \mathrm{XCH}_{3}{ }^{\mathrm{Piv}}\right), 1.08$ (s, 9H, $\left.3 \mathrm{xCH}_{3}{ }^{\mathrm{Piv}}\right), 1.06$ (s, 9H, $\left.3 \mathrm{xCH}_{3}{ }^{\mathrm{Piv}}\right), 1.04$ (s, 9H, 3xCH$\left.{ }_{3}{ }^{\mathrm{Piv}}\right), 1.03$ (s, $\left.18 \mathrm{H}, 6 \mathrm{xCH}_{3}{ }^{\mathrm{Piv}}\right)$, $1.01\left(\mathrm{~s}, 9 \mathrm{H}, 3 \mathrm{xCH}_{3}{ }^{\mathrm{Piv}}\right), 1.00\left(\mathrm{~s}, 9 \mathrm{H}, 3 \mathrm{xCH}_{3}{ }^{\mathrm{Piv}}\right), 1.00(\mathrm{~s}, 9 \mathrm{H}$, $\left.3 \mathrm{xCH}_{3}{ }^{\mathrm{Piv}}\right), 0.99$ (s, 9H, 3xCH $\left.3{ }^{\mathrm{Piv}}\right), 0.97$ (s, 9H, 3xCH $\left.3{ }^{\mathrm{Piv}}\right) .{ }^{13} \mathbf{C} \mathbf{N M R}$ $\left(101 \mathrm{MHz}, \mathrm{CDCl}_{3}\right) \delta 178.32,178.26,176.9,176.8,176.5,176.2$, 176.2, 176.1, 176.0 (2C), 175.7, 138.9, 138.2, 138.03, 138.00, 137.98, 137.9, 137.8, 137.6 (2C), 137.4, 129.2-125.4 (50C), 102.6, 100.7, 100.6, 100.5, 100.4, 100.3 (4C), 100.2 (3C), 100.0, 99.9,
99.7, 99.6, 99.3, 77.4, 76.0, 75.9, 75.8, 75.7, 75.5, 75.2, 74.8, 74.4, 74.2, 73.23, 73.15, 73.0, 72.6, 72.3, 72.1, 72.0, 71.9, 71.7, 71.6, 71.5, 71.4, 71.3, 71.1, 69.9, 69.2, 69.0, 68.93, 68.88, 68.8, 68.6, 68.4, 68.0, 67.6, 67.5, 67.3, 67.0, 66.9, 66.9, 66.8, 66.7, 65.2, 39.1, 39.03, 39.01, 38.83, 38.80, 38.79, 38.77, 38.71 (3C), 38.68, 38.67, 27.5 (3C), 27.4 (6C), 27.29 (3C), 27.27 (3C), 27.25 (3C), 27.20 (3C), 27.15 (6C), 27.12 (3C), 27.10 (3C).

Benzyl 4,6-O-benzylidene-2,3-di-O-pivaloyl- $\beta$-D-galactopyranosyl-( $1 \rightarrow 3$ )-4,6-O-benzylidene-2-O-pivaloyl- $\beta$-D-galactopyranosyl-( $\rightarrow 3)$-[4,6-O-benzylidene-2,3-di- $O$-pivaloyl- $\beta$-D-galactopyranosyl-( $\rightarrow 3)-4,6-O$-benzylidene-2- $O$-pivaloyl- $\beta$-D-galactopyranosyl-( $1 \rightarrow 6)]$-4-O-benzyl-2-O-pivaloyl- $\beta$-D-galactopyranosyl- $(1 \rightarrow 3)-4,6$ - $O$-benzylidene-2- $O$-pivaloyl- $\beta$-D-galactopyranosyl-( $1 \rightarrow 3)-4,6$-Obenzylidene-2-O-pivaloyl- $\beta$-D-galactopyranosyl-( $1 \rightarrow 3)-4,6$ - $O$-benzylidene-2-O-pivaloyl- $\beta$-D-galactopyranosyl-( $1 \rightarrow 3)$-4,6-O-benzylidene-2-O-pivaloyl- $\beta$-D-galactopyranoside (39). To a $25 \mathrm{~mL}$ flame-dried flask was added 33 (500 mg, $0.20 \mathrm{mmol}$ ) and the 20 (255 $\mathrm{mg}, 0.30 \mathrm{mmol})$. The mixture was dried azeotropically with tol-uene $(2 \times 5 \mathrm{~mL})$ and subject-ted to vacuum overnight. It was then dissolved in dry $\mathrm{CH}_{2} \mathrm{Cl}_{2}(2 \mathrm{~mL})$ and dry MeCN (2 mL), cooled to $-30^{\circ} \mathrm{C}$, followed by addition of NIS (69 mg; $0.31 \mathrm{mmol}$ ) and TESOTf (10 mg; $0.04 \mathrm{mmol})$. The reaction mixture was stirred at $-30{ }^{\circ} \mathrm{C}$ until TLC revealed full conversion of the donor $(1 \mathrm{~h})$. The solution was diluted with $\mathrm{CH}_{2} \mathrm{Cl}_{2}$ $(50 \mathrm{~mL})$ and washed with sat. aq. $\mathrm{NaS}_{2} \mathrm{O}_{3}(50 \mathrm{~mL})$ and sat. aq. $\mathrm{NaHCO}_{3}(50 \mathrm{~mL})$. The organic phase was dried over $\mathrm{MgSO}_{4}$, filtered and concentrated. The product was purified by flash chromatography (19:1 Tol/EtOAc) to afford a white solid. Yield: $460 \mathrm{mg}$ (71\%). IR (neat, $\mathrm{cm}^{-1}$ ): 2973.10, 2933.38, 2906.81, 2872.23, 1736.16, 1701.44, 1479.68, 1455.34, 1397.62, 1366.36, 1276.86, 1171.55, 1132.07, 1085.98, 1046.35, 1026.49, 999.26. ${ }^{\mathbf{1}} \mathbf{H}$ NMR (400 MHz, $\left.\mathrm{CDCl}_{3}\right) \delta 7.42(\mathrm{~m}, 16 \mathrm{H}, \mathrm{Ar}-\mathrm{H}), 7.23(\mathrm{~m}, 34 \mathrm{H}, \mathrm{Ar}-\mathrm{H})$, $5.57-5.07$ (m, 17H, H-2 $\left.{ }^{1}, \mathrm{H}-2^{7}-\mathrm{H}-2^{1}, \mathrm{H}-2^{2}, 8 x \mathrm{CH}^{\text {benzylidene }}\right), 4.88$ $-4.81\left(\mathrm{~m}, 3 \mathrm{H}, 2 \mathrm{xH}-1,0.5 \mathrm{xCH}_{2}{ }^{\mathrm{Bn}}\right), 4.79\left(\mathrm{~d}, J_{\mathrm{CH} 2}=11.5 \mathrm{~Hz}, 1 \mathrm{H}\right.$, $0.5 \times \mathrm{CH}_{2}{ }^{\mathrm{Bn}}$ ), $4.74-4.66$ (m, 3H, H- $\left.3^{7 / 2^{\prime}}, \mathrm{H}-3^{7 / 2}, \mathrm{H}-1\right), 4.65$ (d, $J_{1,2}$ $=7.9 \mathrm{~Hz}, 1 \mathrm{H}, \mathrm{H}-1), 4.61\left(\mathrm{~d}, J_{1,2}=7.9 \mathrm{~Hz}, 1 \mathrm{H}, \mathrm{H}-1\right), 4.55$ (d, $J_{1,2}=$ $7.9 \mathrm{~Hz}, 1 \mathrm{H}, \mathrm{H}-1), 4.53\left(\mathrm{~d}, J_{\mathrm{CH} 2}=11.7 \mathrm{~Hz}, 1 \mathrm{H}, 0.5 \mathrm{xCH}_{2}{ }^{\mathrm{Bn}}\right), 4.47(\mathrm{~d}$, $\left.J_{1,2}=7.9 \mathrm{~Hz}, 1 \mathrm{H}, \mathrm{H}-1\right), 4.45\left(\mathrm{~d}, J_{\mathrm{CH} 2}=11.6 \mathrm{~Hz}, 1 \mathrm{H}, \mathrm{CH}_{2}{ }^{\mathrm{Bn}}\right), 4.36$ (d, $\left.J_{1,2}=7.9 \mathrm{~Hz}, 1 \mathrm{H}, \mathrm{H}-1\right), 4.33-3.84\left(\mathrm{~m}, 31 \mathrm{H}, \mathrm{H}-3^{1}-\mathrm{H}-3^{6}, \mathrm{H}-3^{1}{ }^{1}\right.$, $\left.8 \times{ }^{1 / 2 / 3 / 4 / 6 / 7 / 1^{\prime} / 2^{\prime}}, 8 \times H-6^{1 / 2 / 3 / 4 / 6 / 7 / 1^{\prime} / 2^{\prime}}\right), 3.89\left(\mathrm{~d}, J_{1,2}=7.8 \mathrm{~Hz}, 1 \mathrm{H}, \mathrm{H}-\right.$ $1^{1}$ ), 3.82 (m, $1 \mathrm{H}, \mathrm{H}-6 \mathrm{a}^{5}$ ), 3.78 (t, $J_{3,4}=3.4 \mathrm{~Hz}, 1 \mathrm{H}, \mathrm{H}-4^{5}$ ), $3.69-$ $3.53\left(\mathrm{~m}, 1 \mathrm{H}, \mathrm{H}-6 \mathrm{~b}^{5}\right), 3.47$ (d, $\left.J_{5,6 a}=J_{5,6 b}=5.3 \mathrm{~Hz}, 1 \mathrm{H}, \mathrm{H}-5^{5}\right), 3.38$ - 3.35 (m, $2 \mathrm{H}, 2 \times H-5^{1 / 2 / 3 / 4 / 6 / 7 / 1^{\prime} / 2^{\prime}}$ ), 3.30 (s, $1 \mathrm{H}, \mathrm{H}-5^{1 / 2 / 3 / 4 / 6 / 7 / 1^{\prime} / 2^{\prime}}$ ), 3.24 (s, $\left.1 \mathrm{H}, \mathrm{H}-5^{1 / 2 / 3 / 4 / 6 / 7 / 1^{\prime} / 2^{\prime}}\right), 3.23$ (s, $\left.1 \mathrm{H}, \mathrm{H}-5^{1 / 2 / 3 / 4 / 6 / 7 / 1^{\prime} / 2^{\prime}}\right), 3.21$ (s, $1 \mathrm{H}, \mathrm{H}-5^{1 / 2 / 3 / 4 / 6 / 7 / 1^{\prime} / 2^{\prime}}$ ), 3.17 (s, $\left.1 \mathrm{H}, \mathrm{H}-51 / 2 / 3 / 4 / 6 / 7 / 1^{\prime} / 2^{\prime}\right), 3.00$ (s, $1 \mathrm{H}, \mathrm{H}-5^{1 / 2 / 3 / 4 / 6 / 7 / 1^{\prime} / 2^{\prime}}$ ), 1.11 (s, $\left.9 \mathrm{H}, 3 \mathrm{xCH}_{3}{ }^{\mathrm{Piv}}\right), 1.08$ (s, 9H, $3 \mathrm{xCH}_{3}{ }^{\mathrm{Piv}}$ ), $1.07\left(\mathrm{~s}, 27 \mathrm{H}, 9 \mathrm{xCH}_{3}{ }^{\mathrm{Piv}}\right), 1.04\left(\mathrm{~s}, 9 \mathrm{H}, 3 \mathrm{xCH}_{3}{ }^{\mathrm{Piv}}\right), 1.02(\mathrm{~s}, 9 \mathrm{H}$, $\left.3 \mathrm{xCH}_{3}{ }^{\mathrm{Piv}}\right), 1.00$ (s, 9H, $\left.3 \mathrm{xCH}_{3}{ }^{\mathrm{Piv}}\right), 0.98$ (s, 9H, 3xCH$\left.{ }^{\mathrm{Piv}}\right), 0.98$ (s, 9H, $3 \mathrm{xCH}_{3}{ }^{\mathrm{Piv}}$ ), 0.95 (s, 9H, 3xCH${ }^{\mathrm{Piv}}$ ). ${ }^{13} \mathrm{C}$ NMR $(101 \mathrm{MHz}$, $\left.\mathrm{CDCl}_{3}\right) \delta 178.3,178.2177 .0,176.9,176.34,176.31,176.10$, 176.07, 176.01, 175.97, 175.7, 138.9, 138.1, 138.03, 137.98 (2C), 137.9, 137.7, 137.6 (2C), 137.3, 129.1-125.4 (34C), 102.4, 100.7, 100.5 (2C), 100.4, 100.3, 100.3 (3C), 100.2, 100.1 (2C), 99.8, 99.7, 99.6, 99.5, 99.2, 77.4, 76.0, 75.91, 75.85, 75.7, 75.3, 75.2, 74.7, 74.4, 74.2, 73.23, 73.15, 73.0, 72.5, 72.4, 72.03, 71.99, 71.9, 71.5, $71.2,71.1,71.0,70.1,69.2,68.9,68.8,68.6,68.4,68.3,68.0,67.6$, 67.5, 67.4, 67.0, 66.91, 66.87, 66.8, 66.7, 39.02, 39.00, 38.79 (2C), 38.77 (3C), 38.71, 38.69, 38.66, 38.6, 27.40 (6C), 27.36 (3C), 27.30 (3C), 27.29 (3C), 27.22 (3C), 27.21 (3C), 27.15 (3C), 27.12 (6C), 27.09 (3C).

$\beta$-D-Galactopyranosyl-(1 $\rightarrow$ 3)- $\beta$-D-galactopyranosyl- $(1 \rightarrow 3)-\beta$ D-galactopyranosyl-( $1 \rightarrow 3)$ - $\beta$-D-galactopyranosyl-( $1 \rightarrow 3)$-D-galactopyranose (1). 24 (350 mg; $0.18 \mathrm{mmol}$ ) was dissolved in THF (40 mL) and a $1 \mathrm{M} \mathrm{Et} 4 \mathrm{NOH}$ in $\mathrm{MeOH}$ solution $(7.4 \mathrm{~mL} ; 7.4$ mmol) was added. The reaction mixture was stirred at $65^{\circ} \mathrm{C}$ for 
$24 \mathrm{~h}$. The reaction mixture was poured into sat. aq. $\mathrm{NH}_{4} \mathrm{Cl}(100$ $\mathrm{mL}$ ) and extracted with $\mathrm{CH}_{2} \mathrm{Cl}_{2}(3 \times 100 \mathrm{~mL})$. The combined organic phases were dried over $\mathrm{MgSO}_{4}$, filtered and concentrated. The product was purified by flash chromatography (9:1 $\mathrm{CH}_{2} \mathrm{Cl}_{2} / \mathrm{MeOH}$ ) to yield colorless crystals. The product was dissolved in $\mathrm{MeOH}(40 \mathrm{~mL})$, THF (10 mL). 20\% Pd(OH) $/$ /C (129 mg; $0.18 \mathrm{mmol}$ ) was added and an atmosphere of $\mathrm{H}_{2}$ (1 atm.) was installed. The reaction was stirred at $22{ }^{\circ} \mathrm{C}$ for $48 \mathrm{~h}$, filtered through celite, and concentrated to give a grey solid. Purified by reverse phase chromatography to afford $\mathbf{1}$ as a white solid. Yield: $119 \mathrm{mg}$ (78\% over two steps). ${ }^{1} \mathbf{H}$ NMR (800 MHz, D $\mathrm{O}$ ) $\delta 5.19$ (d, $J_{3,4}=3.1 \mathrm{~Hz}, 1 \mathrm{H}, \mathrm{H}-1 \alpha$ ), $4.62-4.49$ (m, $9 \mathrm{H}, \mathrm{H}-1^{1} \beta, \mathrm{H}-1^{5} \beta, \mathrm{H}-$ $1^{2} \alpha, \mathrm{H}-1^{5} \alpha$ ), 4.17 (d, $\left.J_{3,4}=3.4 \mathrm{~Hz}, 1 \mathrm{H}, \mathrm{H}-4\right), 4.11$ (m, 9H, 9xH4), 4.03 (t, $J=6.3 \mathrm{~Hz}, 1 \mathrm{H}), 3.90(\mathrm{t}, J=3.0 \mathrm{~Hz}, 1 \mathrm{H}), 3.83$ (d, $J=$ $3.3 \mathrm{~Hz}, 3 \mathrm{H}), 3.80-3.47$ (m, 45H). ${ }^{13} \mathbf{C}$ NMR (201 MHz, $\left.\mathrm{D}_{2} \mathrm{O}\right) \delta$ 104.4, 104.2, 104.2, 104.1, 96.3, 92.3, 82.6, 82.1, 82.1, 79.5, 75.2, 74.9, 74.8, 74.7, 72.6, 71.1, 71.0, 70.4, 70.3, 70.2, 69.3, 68.7, 68.7, 68.5, 68.4, 67.5, 61.2, 61.1, 61.0, 61.0. HRMS (MALDI) $\mathrm{m} / \mathrm{z}$ : $[\mathrm{M}+\mathrm{H}]^{+}$Calcd for $\mathrm{C}_{30} \mathrm{H}_{53} \mathrm{O}_{26} 829.2820$ Found 829.2841.

$\beta$-D-Galactopyranosyl-( $\rightarrow 3)-\beta$-D-galactopyranosyl-(1 $\rightarrow 3)-\beta$ D-galactopyranosyl- $(1 \rightarrow 3)-\beta$-D-galactopyranosyl- $(1 \rightarrow 3)-\beta$-Dgalactopyranosyl-( $\rightarrow 3)$ - $\beta$-D-galactopyranosyl- $(1 \rightarrow 3)$-D-galactopyranose (2). 26 (325 mg; $0.13 \mathrm{mmol}$ ) was dissolved in THF $(40 \mathrm{~mL})$ and a $1 \mathrm{M} \mathrm{Et} 4 \mathrm{NOH}$ in $\mathrm{MeOH}$ solution $(7.6 \mathrm{~mL} ; 7.6$ $\mathrm{mmol})$ was added. The reaction mixture was stirred at $65^{\circ} \mathrm{C}$ for $24 \mathrm{~h}$. The reaction mixture was poured into sat. aq. $\mathrm{NH}_{4} \mathrm{Cl}(100$ $\mathrm{mL})$ and extracted with $\mathrm{CH}_{2} \mathrm{Cl}_{2}(3 \times 100 \mathrm{~mL})$. The combined organic phases were dried over MgSO4, filtered and concentrated. The product was purified by flash chromatography (9:1 $\mathrm{CH}_{2} \mathrm{Cl}_{2} / \mathrm{MeOH}$ ) to yield colorless crystals. The product was dissolved in $\mathrm{MeOH}$ (40 mL), THF (10 mL). 20\% Pd(OH)2/C (88 mg; $0.13 \mathrm{mmol}$ ) was added and an atmosphere of $\mathrm{H}_{2}$ (1 atm.) was installed. The reaction was stirred at $22{ }^{\circ} \mathrm{C}$ for $48 \mathrm{~h}$, filtered through celite, and concentrated to give a grey solid. Purified by reverse phase chromatography to afford 2 as a white solid. Yield: $107 \mathrm{mg}$ (74\% over two steps). ${ }^{\mathbf{1}} \mathbf{H}$ NMR (800 MHz, D $\left.2 \mathrm{O}\right) \delta 5.19$ (d, $J_{1,2}=$ $3.0 \mathrm{~Hz}, 1 \mathrm{H}, \mathrm{H}-1 \alpha), 4.63-4.48$ (m, $13 \mathrm{H}, \mathrm{H}-1^{1} \beta, \mathrm{H}-1^{7} \beta, \mathrm{H}-1^{2} \alpha, \mathrm{H}-$ $\left.1^{7} \alpha\right), 4.16\left(\mathrm{~d}, J_{3,4}=3.2 \mathrm{~Hz}, 1 \mathrm{H}\right), 4.11(\mathrm{~m}, 13 \mathrm{H}), 4.03(\mathrm{t}, J=6.3$ Hz, 1H), 3.90 (t, $J=3.0 \mathrm{~Hz}, 2 \mathrm{H}), 3.83$ (d, $J=3.2 \mathrm{~Hz}, 2 \mathrm{H}), 3.80-$ 3.47 (m, 65H). ${ }^{13} \mathrm{C}$ NMR (201 MHz, $\mathrm{D}_{2} \mathrm{O}$ ) $\delta$ 104.4, 104.2, 104.1, 104.1, 96.3, 92.3, 82.5, 82.1, 82.1, 81.8, 79.5, 75.2, 74.9, 74.8, 74.7, 72.6, 71.1, 71.0, 70.4, 70.3, 70.2, 69.3, 68.7, 68.5, 68.4, 67.5, 61.2, 61.1, 61.0. HRMS (ESI) m/z: [M + Na $]^{+}$Calcd for $\mathrm{C}_{42} \mathrm{H}_{72} \mathrm{O}_{36} 1175.3695$ Found 1175.3685.

$\beta$-D-Galactopyranosyl-( $\rightarrow 3)-\beta$-D-galactopyranosyl-(1 $\rightarrow 3)-\beta$ D-galactopyranosyl-( $1 \rightarrow 3)-\beta$-D-galactopyranosyl- $(1 \rightarrow 3)-[\beta-D-$ galactopyranosyl-( $1 \rightarrow 3)$ - $\beta$-D-galactopyranosyl- $(1 \rightarrow 6)]-\beta$-D-galactopyranosyl-( $\rightarrow 3)$ - $\beta$-D-galactopyranosyl-( $\rightarrow 3$ )-D-galactopyranose (3). 37 (380 mg; $0.12 \mathrm{mmol}$ ) was dissolved in THF (30 $\mathrm{mL}$ ) and a $1 \mathrm{M} \mathrm{Et} 4 \mathrm{NOH}$ in $\mathrm{MeOH}$ solution $(9.24 \mathrm{~mL}$; $9.24 \mathrm{mmol}$ ) was added. The reaction mixture was stirred at $65{ }^{\circ} \mathrm{C}$ for $24 \mathrm{~h}$. The reaction mixture was poured into sat. aq. $\mathrm{NH}_{4} \mathrm{Cl}(100 \mathrm{~mL})$ and extracted with $\mathrm{CH}_{2} \mathrm{Cl}_{2}(3 \times 100 \mathrm{~mL})$. The combined organic phases were dried over $\mathrm{MgSO}_{4}$, filtered and concentrated. The product was purified by flash chromatography $\left(9: 1 \mathrm{CH}_{2} \mathrm{Cl}_{2} / \mathrm{MeOH}\right)$ to yield colorless crystals. The product was dissolved in $\mathrm{MeOH}(40 \mathrm{~mL}), \mathrm{THF}$ (10 mL). 20\% $\mathrm{Pd}(\mathrm{OH})_{2} / \mathrm{C}$ (84 mg; $0.12 \mathrm{mmol}$ ) was added and an atmosphere of $\mathrm{H}_{2}(1 \mathrm{~atm}$.) was installed. The reaction was stirred at $22{ }^{\circ} \mathrm{C}$ for $48 \mathrm{~h}$, filtered through celite, and concentrated to give a grey solid. Purified by reverse phase chromatography to afford 3 as a white solid. Yield: $112 \mathrm{mg}$ (66\% over two steps). ${ }^{1} \mathbf{H}$ NMR $\left(800 \mathrm{MHz}, \mathrm{D}_{2} \mathrm{O}\right) \delta 5.29$ (d, $\left.J_{1,2}=3.1 \mathrm{~Hz}, 1 \mathrm{H}, \mathrm{H}-1 \alpha\right), 4.73-4.58$ (m, $15 \mathrm{H}, \mathrm{H}-1^{1} \beta, \mathrm{H}-1^{7} \beta, \mathrm{H}-1^{2} \alpha, \mathrm{H}-1^{7} \alpha, \mathrm{H}-1^{\prime}$ branch $\alpha, \mathrm{H}-1^{\prime 6 b r a n c h} \beta$ ), 4.49 (d, $J_{1,2}=7.8 \mathrm{~Hz}, 2 \mathrm{H}, \mathrm{H}-1^{\text {branch }} \alpha, \mathrm{H}-1^{\text {branch }} \beta$ ), 4.26 (d, $J_{3,4}=$ $\left.3.3 \mathrm{~Hz}, 1 \mathrm{H}, \mathrm{H}-4^{1} \alpha\right), 4.22(\mathrm{~m}, 17 \mathrm{H}, 17 \mathrm{xH}-4), 4.12(\mathrm{t}, J=6.3 \mathrm{~Hz}$, 1H), $4.08-3.55$ (m, 89H). ${ }^{13}$ C NMR (201 MHz, D $\left.{ }_{2} \mathrm{O}\right) \delta 104.5$, 104.4, 104.2, 104.1, 104.0, 103.9, 103.2, 96.2, 92.2, 82.65, 82.6, 82.5, 82.1, 82.05, 82.0, 81.95, 81.9, 81.8, 79.6, 75.1, 74.8, 74.8, 74.8, 73.4, 72.6, 71.1, 71.1, 71.0, 70.3, 70.2, 70.2, 69.9, 69.3, 69.2, 68.6, 68.5, 68.4, 68.4, 68.4, 67.4, 61.2, 61.0, 60.9. HRMS (MALDI) $\mathrm{m} / \mathrm{z}:[\mathrm{M}+\mathrm{Na}]^{+}$Calcd for $\mathrm{C}_{54} \mathrm{H}_{92} \mathrm{O}_{46} \mathrm{Na} 1499.4752$ Found 1499.4780 .

$\beta$-D-Galactopyranosyl-(1 $\rightarrow 3)$ - $\beta$-D-galactopyranosyl- $(1 \rightarrow 3)$-[ $\beta$ D-galactopyranosyl- $(1 \rightarrow 3)-\beta$-D-galactopyranosyl- $(1 \rightarrow 6)]-\beta$-Dgalactopyranosyl- $(1 \rightarrow 3)-\beta$-D-galactopyranosyl- $(1 \rightarrow 3)-\beta$-D-galactopyranosyl-( $1 \rightarrow 3)$ - $\beta$-D-galactopyranosyl-( $\rightarrow 3)$-D-galactopyranose (4). 39 (318 $\mathrm{mg} ; 0.097 \mathrm{mmol}$ ) was dissolved in THF $(30 \mathrm{~mL})$ and a $1 \mathrm{M} \mathrm{Et} 4 \mathrm{NOH}$ in $\mathrm{MeOH}$ solution $(7.7 \mathrm{~mL} ; 7.7 \mathrm{mmol}$ ) was added. The reaction mixture was stirred at $65^{\circ} \mathrm{C}$ for $24 \mathrm{~h}$. The reaction mixture was poured into sat. aq. $\mathrm{NH}_{4} \mathrm{Cl}(100 \mathrm{~mL})$ and extracted with $\mathrm{CH}_{2} \mathrm{Cl}_{2}(3 \times 100 \mathrm{~mL})$. The combined organic phases were dried over MgSO4, filtered and concentrated. The product was purified by flash chromatography $\left(9: 1 \mathrm{CH}_{2} \mathrm{Cl}_{2} / \mathrm{MeOH}\right)$ to yield colorless crystals. The product was dissolved in $\mathrm{MeOH}(40 \mathrm{~mL})$, THF (10 mL). 20\% Pd(OH)2/C (68 mg; $0.097 \mathrm{mmol})$ was added and an atmosphere of $\mathrm{H}_{2}(1 \mathrm{~atm}$.) was installed. The reaction was stirred at $22^{\circ} \mathrm{C}$ for $48 \mathrm{~h}$, filtered through celite, and concentrated to give a grey solid. Purified by reverse phase chromatography to afford 4 as a white solid. Yield: $102 \mathrm{mg}$ (72\% over two steps). ${ }^{1} \mathbf{H}$ NMR (800 MHz, D $2 \mathrm{O}) \delta 5.28$ (d, $\left.J_{1,2}=3.0 \mathrm{~Hz}, 1 \mathrm{H}, \mathrm{H}-1 \alpha\right)$, $4.73-$ 4.58 (m, $15 \mathrm{H}, \mathrm{H}-1^{1} \beta, \mathrm{H}-1^{7} \beta, \mathrm{H}-1^{2} \alpha, \mathrm{H}-1^{7} \alpha, \mathrm{H}-1^{\text {'branch }} \alpha, \mathrm{H}-1^{\text {'branch }} \beta$ ), 4.49 (d, $\left.J_{1,2}=7.9 \mathrm{~Hz}, 3 \mathrm{H}, \mathrm{H}-1^{\text {branch }} \alpha, \mathrm{H}-1^{\text {branch }} \beta\right), 4.26$ (d, $J_{3,4}=3.1$

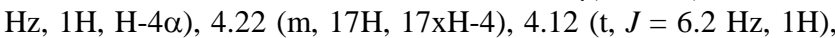
4.09 - 3.56 (m, 89H). ${ }^{13} \mathrm{C}$ NMR (201 MHz, D $\left.2 \mathrm{O}\right) \delta 104.5,104.4$, 104.1, 104.1, 104.0, 103.9, 103.2, 96.2, 92.2, 82.6, 82.5, 82.1, 82.0, 82.0, 81.9, 79.4, 75.1, 74.8, 74.8, 74.7, 74.7, 73.4, 72.6, 71.1, 71.1, 71.0, 70.3, 70.3, 70.2, 70.2, 70.2, 69.9, 69.3, 69.2, 68.6, 68.5, 68.4, 68.4, 67.4, 61.2, 61.0, 60.9. HRMS (MALDI) m/z: [M + H] ${ }^{+}$Calcd for $\mathrm{C}_{54} \mathrm{H}_{93} \mathrm{O}_{46} 1477.4933$ Found 1477.4953.

$\beta$-D-Galactopyranosyl-(1 $\rightarrow 3)$ - $\beta$-D-galactopyranosyl- $(1 \rightarrow 3)$-[ $\beta$ D-galactopyranosyl- $(1 \rightarrow 6)-\beta$-D-galactopyranosyl- $(1 \rightarrow 6)]-\beta$-Dgalactopyranosyl- $(1 \rightarrow 3)$ - $\beta$-D-galactopyranosyl- $(1 \rightarrow 3)-\beta$-D-galactopyranosyl- $(1 \rightarrow 3)$ - $\beta$-D-galactopyranosyl-( $\rightarrow 3)$-D-galactopyranose (5). 40 (328 mg; $0.097 \mathrm{mmol}$ ) was dissolved in THF $(30 \mathrm{~mL})$ and a $1 \mathrm{M} \mathrm{Et} 4 \mathrm{NOH}$ in $\mathrm{MeOH}$ solution $(7.78 \mathrm{~mL}$; $7.78 \mathrm{mmol}$ ) was added. The reaction mixture was stirred at $65{ }^{\circ} \mathrm{C}$ for $24 \mathrm{~h}$. The reaction mixture was poured into sat. aq. $\mathrm{NH}_{4} \mathrm{Cl}$ $(100 \mathrm{~mL})$ and extracted with $\mathrm{CH}_{2} \mathrm{Cl}_{2}(3 \times 100 \mathrm{~mL})$. The combined organic phases were dried over $\mathrm{MgSO}_{4}$, filtered and concentrated. The product was purified by flash chromatography (9:1 $\mathrm{CH}_{2} \mathrm{Cl}_{2} / \mathrm{MeOH}$ ) to yield a colorless foam. The product was dissolved in $\mathrm{MeOH}$ (30 mL), THF (7.5 mL). 20\% Pd(OH)2/C (68 mg; $0.097 \mathrm{mmol}$ ) was added and an atmosphere of $\mathrm{H}_{2}$ (1 atm.) was installed. The reaction was stirred at $22{ }^{\circ} \mathrm{C}$ for $48 \mathrm{~h}$, filtered through celite, and concentrated to give a grey solid. Purified by reverse phase chromatography to afford 5 as a white solid. Yield: 99 mg (69\% over two steps). ${ }^{1} \mathbf{H}$ NMR (800 MHz, D $\left.2 \mathrm{O}\right) \delta 5.19$ (d, $J_{1,2}=$ $3.1 \mathrm{~Hz}, 1 \mathrm{H}, \mathrm{H}-1 \alpha$ ), $4.64-4.48$ (m, $13 \mathrm{H}, \mathrm{H}-1^{1} \beta, \mathrm{H}-1^{7} \beta, \mathrm{H}-1^{2} \alpha, \mathrm{H}-$ $1^{7} \alpha$ ), 4.36 (d, $J_{1,2}=7.8 \mathrm{~Hz}, 2 \mathrm{H}, \mathrm{H}-1^{\text {branch }} \alpha, \mathrm{H}-1^{6 \text { branch }} \beta$ ), 4.35 (d, $J_{1,2}=7.8 \mathrm{~Hz}, 2 \mathrm{H}, \mathrm{H}-1^{\prime \text { branch }} \alpha, \mathrm{H}-1^{\prime \text { b branch }} \beta$ ), $4.19-4.08$ (m, $18 \mathrm{H}$, $18 \mathrm{xH}-4), 4.03$ (t, $J=6.3 \mathrm{~Hz}, 1 \mathrm{H}), 3.98-3.38$ (m, 89H). ${ }^{13} \mathbf{C}$ NMR (201 MHz, D2O) $\delta 104.4,104.2,104.1,104.1,104.1,103.9,103.6$, 103.5, 96.2, 92.2, 82.5, 82.1, 82.0, 82.0, 81.8, 79.5, 75.2, 75.1, 74.9, 74.8, 74.8, 74.7, 73.8, 73.3, 72.8, 72.7, 72.6, 71.1, 71.0, 70.8, 70.8, 70.3, 70.3, 70.3, 70.2, 69.5, 69.4, 69.2, 68.7, 68.6, 68.5, 68.4, 67.5, 61.2, 61.1, 61.0, 61.00, 61.0. HRMS (MALDI) m/z: $[\mathrm{M}+\mathrm{H}]^{+}$ Calcd for $\mathrm{C}_{54} \mathrm{H}_{93} \mathrm{O}_{46}$ 1477.4933 Found 1477.4956. 
$\beta$-D-Galactopyranosyl-(1 $\rightarrow 3)$ - $\beta$-D-galactopyranosyl-( $1 \rightarrow 3)$ - $[\alpha$ L-arabinosyl-( $1 \rightarrow 6)]$ - $\beta$-D-galactopyranosyl- $(1 \rightarrow 3)$ - $\beta$-D-galactopyranosyl-( $1 \rightarrow 3)$ - $\beta$-D-galactopyranosyl- $(1 \rightarrow 3)-\beta$-D-galactopyranosyl-(1 $\rightarrow$ 3)-D-galactopyranose $(\mathbf{6}) .38$ (300 $\mathrm{mg} ; 0.10 \mathrm{mmol}$ ) was dissolved in THF ( $30 \mathrm{~mL})$ and a $1 \mathrm{M} \mathrm{Et} 4 \mathrm{NOH}$ in $\mathrm{MeOH}$ solution $(8.1 \mathrm{~mL} ; 8.1 \mathrm{mmol})$ was added. The reaction mixture was stirred at $65{ }^{\circ} \mathrm{C}$ for $24 \mathrm{~h}$. The reaction mixture was poured into sat. aq. $\mathrm{NH}_{4} \mathrm{Cl}(100 \mathrm{~mL})$ and extracted with $\mathrm{CH}_{2} \mathrm{Cl}_{2}(3 \times 100 \mathrm{~mL})$. The combined organic phases were dried over MgSO4, filtered and concentrated. The product was purified by flash chromatography (9:1 $\left.\mathrm{CH}_{2} \mathrm{Cl}_{2} / \mathrm{MeOH}\right)$ to yield colorless crystals. The product was dissolved in $\mathrm{MeOH}(40 \mathrm{~mL})$, THF (10 mL). 20\% Pd(OH) $/ \mathrm{C}$ (70 mg; $0.097 \mathrm{mmol})$ was added and an atmosphere of $\mathrm{H}_{2}(1 \mathrm{~atm}$.) was installed. The reaction was stirred at $22^{\circ} \mathrm{C}$ for $48 \mathrm{~h}$, filtered through celite, and concentrated to give a grey solid. Purified by reverse phase chromatography to afford $\mathbf{6}$ as a white solid. Yield: $84 \mathrm{mg}$ (65\% over two steps). ${ }^{1} \mathbf{H}$ NMR $\left(800 \mathrm{MHz}, \mathrm{D}_{2} \mathrm{O}\right) \delta 5.29$ (d, $J_{1,2}=$ $3.0 \mathrm{~Hz}, 1 \mathrm{H}, \mathrm{H}-1 \alpha), 5.08-5.04$ (m, $\left.2 \mathrm{H}, \mathrm{H}-1^{\text {ara }} \alpha, \mathrm{H}-1^{\text {ara }} \beta\right), 4.74-$ 4.57 (m, $\left.13 \mathrm{H}, \mathrm{H}-1^{1} \beta, \mathrm{H}-1^{7} \beta, \mathrm{H}-1^{2} \alpha, \mathrm{H}-1^{7} \alpha\right), 4.26$ (d, $J_{3,4}=3.1 \mathrm{~Hz}$, $1 \mathrm{H}, \mathrm{H}-4 \alpha), 4.21$ (m, 13H, 13xH-4), 4.12 (t, $J=6.2 \mathrm{~Hz}, 1 \mathrm{H}), 4.10-$ 3.36 (m, 79H). ${ }^{13} \mathrm{C}$ NMR (201 MHz, D2O) $\delta 108.0,104.4,104.2$, 104.1, 104.1, 104.1, 103.9, 96.3, 92.3, 84.0, 82.5, 82.2, 82.1, 82.1, 82.0, 81.2, 79.5, 76.6, 75.2, 74.9, 74.8, 73.5, 72.6, 71.1, 71.0, 70.3, 70.2, 69.3, 68.7, 68.6, 68.6, 68.5, 68.4, 67.5, 67.5, 61.3, 61.2, 61.1, 61.0. HRMS (MALDI) m/z: $[\mathrm{M}+2 \mathrm{Na}]^{+}$Calcd for $\mathrm{C}_{47} \mathrm{H}_{80} \mathrm{O}_{40} \mathrm{Na} 2$ 665.2005 Found 665.2018.

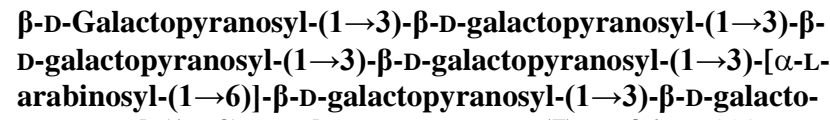
pyranosyl-(1 $\rightarrow$ 3)-D-galactopyranose $\quad$ (7). $36 \quad(320 \quad \mathrm{mg}$; $0.11 \mathrm{mmol}$ ) was dissolved in THF (30 mL) and a $1 \mathrm{M} \mathrm{Et}_{4} \mathrm{NOH}$ in MeOH solution $(9.24 \mathrm{~mL} ; 9.24 \mathrm{mmol})$ was added. The reaction mixture was stirred at $65{ }^{\circ} \mathrm{C}$ for $24 \mathrm{~h}$. The reaction mixture was poured into sat. aq. $\mathrm{NH}_{4} \mathrm{Cl}(100 \mathrm{~mL})$ and extracted with $\mathrm{CH}_{2} \mathrm{Cl}_{2}$ (3x100 mL). The combined organic phases were dried over $\mathrm{MgSO}_{4}$, filtered and concentrated. The product was purified by flash chromatography $\left(9: 1 \mathrm{CH}_{2} \mathrm{Cl}_{2} / \mathrm{MeOH}\right)$ to yield a colorless foam. The product was dissolved in $\mathrm{MeOH}(30 \mathrm{~mL})$, THF $(7.5 \mathrm{~mL})$. $20 \% \mathrm{Pd}(\mathrm{OH})_{2} / \mathrm{C}$ (75 mg; $0.11 \mathrm{mmol}$ ) was added and an atmosphere of $\mathrm{H}_{2}\left(1 \mathrm{~atm}\right.$.) was installed. The reaction was stirred at $22{ }^{\circ} \mathrm{C}$ for $48 \mathrm{~h}$, filtered through celite, and concentrated to give a grey solid. Purified by reverse phase chromatography to afford 7 as a white solid. Yield: $82 \mathrm{mg}$ (60\% over two steps). ${ }^{\mathbf{1}} \mathbf{H}$ NMR $(800 \mathrm{MHz}$, $\left.\mathrm{D}_{2} \mathrm{O}\right) \delta 5.19\left(\mathrm{~d}, J_{1,2}=3.1 \mathrm{~Hz}, 1 \mathrm{H}, \mathrm{H}-1 \alpha\right), 4.97$ (m, $2 \mathrm{H}, \mathrm{H}-1^{\mathrm{ara}} \alpha, \mathrm{H}-$ $1^{\text {ara }} \beta$ ), $4.63-4.51$ (m, $\left.11 \mathrm{H}, \mathrm{H}-1^{1} \beta, \mathrm{H}-1^{7} \beta, \mathrm{H}-1^{2} \alpha, \mathrm{H}-1^{7} \alpha\right), 4.16$ (d, $\left.J_{3,4}=3.5 \mathrm{~Hz}, 1 \mathrm{H}, \mathrm{H}-4 \alpha\right), 4.13-4.08(\mathrm{~m}, 13 \mathrm{H}, 13 \times \mathrm{H}-4), 4.03(\mathrm{t}, J=$ $6.2 \mathrm{~Hz}, 1 \mathrm{H}), 4.00-3.92(\mathrm{~m}, 4 \mathrm{H}), 3.90(\mathrm{t}, J=2.9 \mathrm{~Hz}, 2 \mathrm{H}), 3.88-$ 3.46 (m, 69H). ${ }^{13} \mathrm{C}$ NMR (201 MHz, $\left.\mathrm{D}_{2} \mathrm{O}\right) \delta 108.0,104.4,104.2$, 104.2, 104.1, 103.9, 96.3, 92.3, 84.0, 82.6, 82.2, 82.1, 82.1, 82.0, 81.2, 79.5, 76.6, 75.2, 74.9, 74.8, 74.8, 74.8, 73.5, 72.6, 71.1, 71.1, 70.4, 70.3, 70.3, 70.2, 69.3, 68.7, 68.6, 68.6, 68.5, 68.4, 67.5, 67.5, 61.3, 61.2, 61.1, 61.1, 61.0, 61.0. HRMS (MALDI) m/z: [M+ H] ${ }^{+}$ Calcd for $\mathrm{C}_{47} \mathrm{H}_{81} \mathrm{O}_{40} 1285.4299$ Found 1285.4321.

\section{Glycan microarray printing and analysis of JIM16 and JIM133 binding}

The oligosaccharides were diluted in coupling buffer (80\% $50 \mathrm{mM}$ sodium phosphate, pH 8.5, 0.005\% CHAPS, 20\% PEG400 (Roth)) to four concentrations ( $200 \mu \mathrm{M}, 50 \mu \mathrm{M}, 12.5 \mu \mathrm{M}$, and $3.1 \mu \mathrm{M})$, and printed on CodeLink $N$-hydroxyl succinimide (NHS) ester-activated glass slides (SurModics Inc., Eden Prairie, MN, USA) using a non-contact piezoelectric spotting device (S3; Scienion, Berlin, Germany). After printing, the microarray slides were quenched for
$1 \mathrm{~h}$ at room temperature in quenching buffer (100 mM ethanolamine, $50 \mathrm{mM}$ sodium phosphate, $\mathrm{pH}$ 9) and washed three times with deionized water. The monoclonal antibodies JIM16 and JIM133 were obtained from Plant Probes (Leeds, UK) and the Complex Carbohydrate Research Center (CCRC, Athens, Georgia, USA), respectively. We applied a FlexWell 64 grid (Grace BioLabs, Bend, OR, USA) to the slide and blocked the slides with $1 \%$ bovine serum albumin (BSA) in phosphate-buffered saline (PBS) for $1 \mathrm{~h}$ at room temperature. Then, JIM16 and JIM133 hybridoma supernatant was diluted 1:10 in PBS containing 1\% BSA and incubated for $1 \mathrm{~h}$ on the slides. After three washes with PBS, the slides were incubated with the secondary goat anti-rat IgG AF555 antibody (Invitrogen, Carlsbad, CA, USA) for $1 \mathrm{~h}$. After consecutive washes with $0.1 \%$ Tween-20 in PBS, PBS, and deionized water, the slides were dried by centrifugation ( $300 \mathrm{x} \mathrm{g}, 2 \mathrm{~min}$ ), and the fluorescent signal on the slides was scanned with a GenePix 4300A microarray scanner (Molecular Devices, Sunnyvale, CA, USA) as shown in Figure 3. To obtain the percentages of maximal binding as shown in Table 1, the fluorescent signal of the $200 \mu \mathrm{M}$ concentration was quantified using the GenePix Pro 7 software (Molecular Devices) and normalized to the highest value (given the value 100).

\section{ASSOCIATED CONTENT}

\section{Supporting information}

Copies of NMR spectra of the products and intermediates.

\section{ACKNOWLEDGMENT}

We are grateful to Prof. Michael G. Hahn at CCRC and Prof. J. Paul Knox at University of Leeds for providing samples of JIM133 and JIM16, respectively. We acknowledge financial support from the Danish Council for Independent Research (Grant Case no.: 107279), the Villum Foundation (PLANET project) and the Novo Nordisk Foundation (Biotechnology-based Synthesis and Production Research). F. Pfrengle gratefully acknowledges financial support from the Max Planck Society and the German Research Foundation (DFG, Emmy Noether program PF850/1-1). The NMR Center - DTU is acknowledged for access to the 400 and $800 \mathrm{MHz}$ spectrometers.

\section{REFERENCES}

(1) Doblin, M. S.; Pettolino, F.; Bacic, A. Funct. Plant Biol. 2010, 37, 357-381.

(2) Nangia-Makker, P.; Honjo, Y.; Sarvis, R.; Akahani, S.; Hogan, V.; Pienta, K. J.; Raz, A. Am. J. Pathol. 2000, 156, 899.

(3) Schultz, C.; Gilson, P.; Oxley, D.; Youl, J.; Bacic, A. Trends Plant Sci. 1998, 3, 426.

(4) van Hengel, A. J.; Roberts, K. Plant J. 2003, 36, 256.

(5) van Hengel, A. J.; Roberts, K. Plant J. 2002, 32, 105.

(6) Gao, M.; Showalter, A. M. Plant J. 1999, 19, 321.

(7) Knox, J. P. Int. Rev. Cytol. 1997, 171, 79.

(8) Gane, A. M.; Craik, D.; Munro, S. L. A.; Howlett, G. J.; Clarke, A. E.; Bacic, A. Carbohydr. Res. 1995, 277, 67.

(9) Ellis, M.; Egelund, J.; Schultz, C. J.; Bacic, A. Plant Physiol. 2010, 153, 403.

(10) Liwanag, A. J. M.; Ebert, B.; Verhertbruggen, Y.; Rennie, E. A.; Rautengarten, C.; Oikawa, A.; Andersen, M. C. F.; Clausen, M. H.; Scheller, H. V. Plant Cell 2012, 24, 5024.

(11) Clausen, M. H.; Willats, W. G. T.; Knox, J. P. Carbohydr. Res. 2003, 338, 1797.

(12) Andersen, M. C. F.; Boos, I.; Marcus, S. E.; Kračun, S. K.; Rydahl, M. G.; Willats, W. G. T.; Knox, J. P.; Clausen, M. H. Carbohydr. Res. 2016, 436, 36.

(13) Bartetzko, M. P.; Schuhmacher, F.; Hahm, H. S.; Seeberger, P. H.; Pfrengle, F. Org. Lett. 2015, 17, 4344.

(14) Fenger, T. H.; Madsen, R. European J. Org. Chem. 2013, 2013, 
5923.

Kovac, P.; Taylor, R. B.; Glaudemans, C. P. J. J. Org. Chem. 1985, 50, 5323.

(16) McGill, N. W.; Williams, S. J. J. Org. Chem. 2009, 74, 9388.

(17) Lichtenthaler, F. W.; Oberthür, M.; Peters, S. Eur. J. Org. Chem. 2001, 3849.

(18) Andersen, M. C. F.; Kračun, S. K.; Rydahl, M. G.; Willats, W. G. T.; Clausen, M. H. Chem. Eur. J. 2016, 22, 11543.

(19) Li, A.; Kong, F. Carbohydr. Res. 2005, 340, 1949.

(20) Misra, A. K.; Agnihotri, G. Carbohydr. Res. 2004, 339, 885.

(21) Motawia, M. S.; Marcussen, J.; Møller, B. L. J. Carbohydr. Chem. 1995, 14, 1279.

(22) Xu, Y.-C.; Bizuneh, A.; Walker, C. Tetrahedron Lett. 1996, 37, 455.

(23) Neilson, T.; Werstiuk, E. S. Can. J. Chem. 1971, 49 (18), 3004.

(24) Nicolaou, K. C.; Webber, S. E. Synthesis 1986, 453.

(25) Ley, S. V.; Armstrong, A.; Díez-Martín, D.; Ford, M. J.; Grice, P.; Knight, J. G.; Kolb, H. C.; Madin, A.; Marby, C. A.; Mukherjee, S.; Shaw, A. N.; Slawin, A. M. Z.; Vile, S.; White, A. D.; Williams, D. J.; Woods, M. J. Chem. Soc. Perkin Trans. 1 1991, 667.

(26) Shie, C.-R.; Tzeng, Z.-H.; Wang, C.-C.; Hung, S.-C. J. Chinese Chem. Soc. 2009, 56, 510.

(27) Cunico, R. F.; Bedell, L. J. Org. Chem. 1980, 45, 4797.

(28) Pedersen, C. M.; Nordstrøm, L. U.; Bols, M. J. Am. Chem. Soc. 2007, 129, 9222.

(29) Adinolfi, M.; Barone, G.; Guariniello, L.; Iadonisi, A. Tetrahedron Lett. 2000, 41, 9305.

(30) Lakhmiri, R.; Lhoste, P.; Sinou, D. Tetrahedron Lett. 1989, 30, 4669.

(31) David, C.; Smith, M. J. Am. Chem. Soc. 2001, 123, 9015.

(32) Codée, J. D. C.; Litjens, R. E. J. N.; den Heeten, R.; S. Overkleeft, H.; van Boom, J. H.; van der Marel, G. A. Org. Lett. 2003, 5, 1519.

(33) János, T.; Fügedi, P. Org. Lett. 2007, 9, 4647.
Liang, X.-Y.; Deng, L.-M.; Liu, X.; Yang, J.-S. Tetrahedron 2010, 66, 87.

Pedersen, H. L.; Fangel, J. U. McCleary, B.; Ruzanski, C. Rydahl, M. G.; Ralet, M.-C.; Farkas, V.; von Schantz, L.; Marcus, S. E.; Andersen, M. C. F.; Field, R.; Ohlin, M.; Knox, J. P.; Clausen, M. H.; Willats, W. G. T. J. Biol. Chem. 2012, 287, 39429.

Schmidt, D.; Schuhmacher, F.; Geissner, A.; Seeberger, P. H.; Pfrengle, F. Chem. Eur. J. 2015, 21, 5709.

Ruprecht, C.; Bartetzko, M. P.; Senf, D.; Dallabernadina, P.; Andersen, M. C. F.; Boos, I.; Toshihisa, K.; Knox, J. P.; Hahn, M. G.; Clausen, M. H.; Pfrengle, F. Plant Physiol., in press, DOI:10.1104/pp.17.00737

Knox, J. P.; Linstead, P. J.; Cooper, J. P. C.; Roberts, K. Plant J. 1991, 1, 317.

Pattathil, S.; Avci, U.; Baldwin, D.; Swennes, A. G.; McGill, J. A.; Popper, Z.; Bootten, T.; Albert, A.; Davis, R. H.; Chennareddy, C.; Dong, R.; O’Shea, B.; Rossi, R.; Leoff, C.; Freshour, G.; Narra, R.; O’Neil, M.; York, W. S.; Hahn, M. G. Plant Physiol. 2010, 153, 514.

Hu, Y.; Qin, Y.; Zhao, J. Protoplasma, 2006, 229, 21-31.

Cannesan, M. A.; Durand, C.; Burel, C.; Gangneux, C.; Lerouge, P.; Ishii, T.; Laval, K.; Follet-Gueye, M.-L.; Driouich, A.; VicréGibouin, M. Plant Physiol. 2012, 159, 1658.

Yates, E. A.; Valdor, J.-F.; Haslam, S. M.; Morris, H. R.; Dell, A.; Mackie, W.; Knox, J. P. Glycobiol. 1996, 6, 131.

Gottlieb, H. E.; Kotlyar, V.; Nudelman, A. J. Org. Chem. 1997, 62, 7512.

Kanaya, T.; Schweizer, F.; Takeda, T.; Kiuchi, F.; Hada, N. Carbohydr. Res. 2012, 361, 55. 